\title{
Activity of phenolic compounds from plant origin against Candida species
}

\author{
Natália Martins ${ }^{\mathrm{a}, \mathrm{b}}$, Lillian Barros ${ }^{\mathrm{a}}$, Mariana Henriques ${ }^{\mathrm{b}}$, Sónia Silva ${ }^{\mathrm{b}}$, \\ Isabel C.F.R. Ferreira ${ }^{a, *}$ \\ a Mountain Research Centre (CIMO), ESA, Polytechnic Institute of Bragança, Campus de Santa Apolónia, Apartado 1172, $5301-855$ Bragança, Portugal \\ ${ }^{\mathrm{b}}$ CEB, Centre of Biological Engineering, LIBRO - Laboratório de Investigação em Biofilmes Rosário Oliveira, University of Minho, $4710-057$ Braga, Portugal
}

\section{A R T I C L E I N F O}

\section{Article history:}

Received 29 December 2014

Received in revised form 19 April 2015

Accepted 27 May 2015

\section{Keywords:}

Phytochemicals

Plant extracts

Phenolic compounds

Antifungal activity

Candida species

\begin{abstract}
A B S T R A C T
Candida albicans and other Candida species have been highly associated with several opportunistic fungal infections. Their ability to develop host infections is incited by different determinants, being virulence factors the most highlighted. Molecular targets of the antifungal drugs are crucial components for determination of yeast survival. Ergosterol, nucleic acids and glucan are the most studied molecular targets to destroy Candida species, being considered the basis of the development of new antifungal drugs. However, increasing levels of resistant Candida species to the current antifungal drugs have been observed, making ineffective those agents. Thus, other therapies more effective and safer than the current ones, are being studied, namely the use plant of extracts enriched in phenolic compounds. In this sense, this manuscript provides an historical perspective of the opportunistic fungal infections, molecular targets of the current anti-Candida drugs, as well as a general description of the active principles present in plants, focused on the antifungal potential of whole plant extracts and isolated phenolic compounds, against Candida species.
\end{abstract}

(C) 2015 Elsevier B.V. All rights reserved.

\section{Contents}

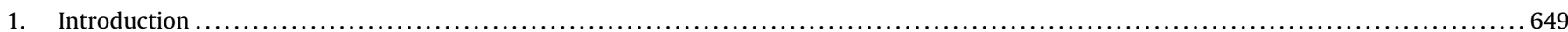

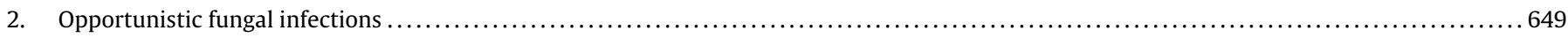

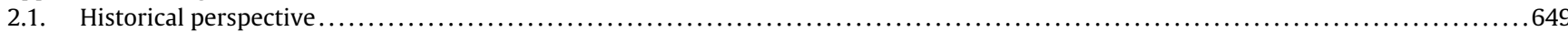

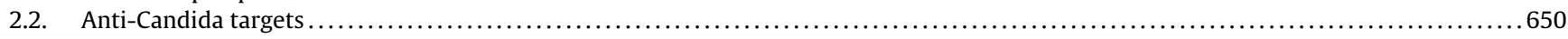

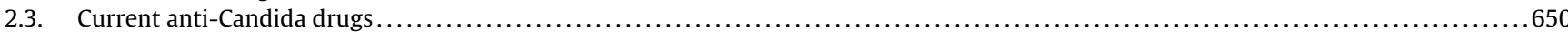

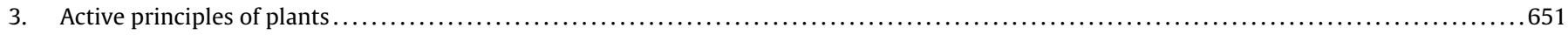

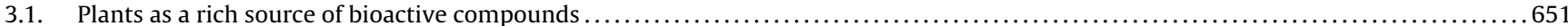

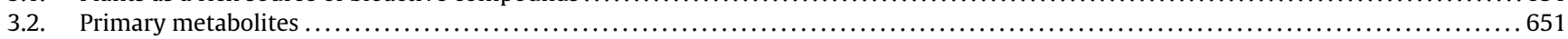

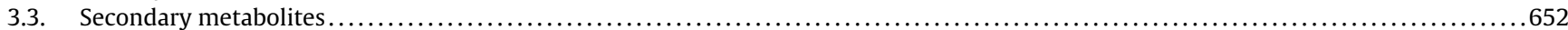

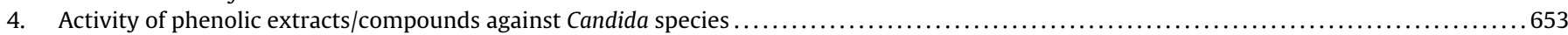

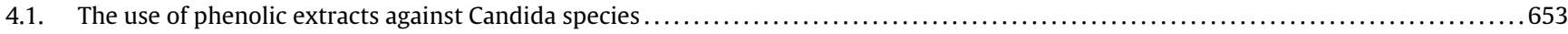

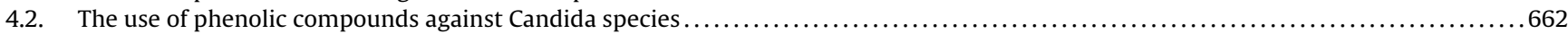

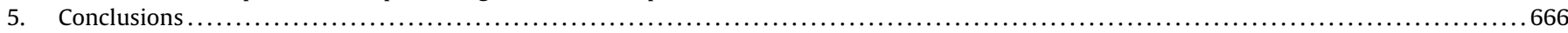

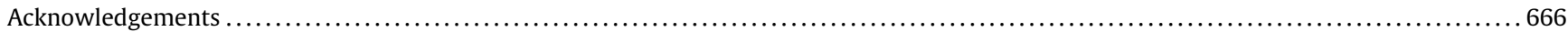

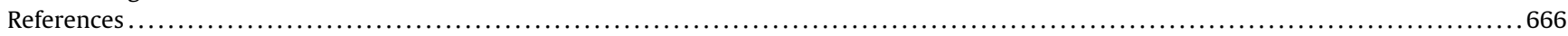

\footnotetext{
* Corresponding author. Tel.: +351 273303219; fax: +351 273325405.

E-mail address: iferreira@ipb.pt (I.C.F.R. Ferreira).
} 


\section{Introduction}

Fungi and other microbial species are widespread in the environment, including the soil, plants, trees, and even in the skin and other parts of the body. Most of them are not dangerous, even conferring some benefits to the host, being considered as commensal flora. Notwithstanding, some species in face to an abnormal overgrowth, might become harmful to the host and, in last instance, could compromise its life (Blanco and Garcia, 2008; Brunke and Hube, 2013).

In the last two decades, it has been observed a considerable increase in the incidence of deep fungal infections, not only in hospital environments, due to the increasing magnitude of organ transplantations, the rise of AIDS, the use of invasive devices (catheters, artificial joints and valves) and in immunocompromised patients, but also in the rest of population (Abi-Said et al., 1997; Eggimann et al., 2003a; Fanello et al., 2001; Kim and Sudbery, 2011; Li et al., 2006; Mayer et al., 2013; Raman et al., 2013; Silva et al., 2011a; Tsai et al., 2013; Wächtler et al., 2012). In parallel with this, and despite the advances on medical and chemical industries increased the life expectancy, due to the indiscriminate use of some chemical substances, it has been observed an increasing resistance of pathogenic microorganisms to conventional drugs, leading to development of other complications (Agarwal et al., 2010; Alves-Silva et al., 2013; Asgarpanah and Kazemivash, 2012; Asl and Hosseinzadeh, 2008; Bakkali et al., 2008; Kanafani and Perfect, 2008; Rana et al., 2011; Sher, 2009; Shojaii and Fard, 2012; Silva et al., 2011b; Singh et al., 2010).

Currently, fungal infections are the fourth leading causes of hematogenous infections and the most common involved fungi are Candida species, commensal microorganisms present in any healthy people (Pierce, 2005; Tsai et al., 2013). Symptoms and signals of fungal infections are not always visible, and an evolution to a systemic infection thrives very slowly, mostly of times, without any mistrust by the individual infected. There are several causal factors that contribute to those opportunistic fungal infections, some of them difficult, or even impossible, to avert. Pollution, smoking, certain drugs, poor diet, sedentary lifestyle and stress-inducing agents are some examples of aggressors (Devasagayam et al., 2004). However, not only external factors increase the organisms vulnerability to disorders, but also genetic changes in the cells, degeneration, reduction of the cells functionality (characteristic of aging process); damage in white blood cells and attacks in other cells of the organic defense system, weak the immune system, leading to a decrease in the ability to respond to invading organisms (Valko et al., 2007).

Natural defense systems are present in living organisms, but it is necessary to consider that in some conditions/situations, an external strengthening and complementation diets are essential (Carocho and Ferreira, 2013a; Devasagayam et al., 2004). It is an established fact that any unbalance affects the well-being of body and potentiates the growing and colonization of certain invaders, among other organic disorders. Thus, a balanced diet, containing all macro and micronutrients in right proportions is crucial to an optimal health, which was already stated by Hippocrates, the father of modern medicine, nearly 2.500 years ago: "Let food be the medicine and medicine be the food". There are some nutrients present in foods that can help to protect and strength the body, at greater or lesser degree (Devasagayam et al., 2004; Murray and Pizzorno, 1998; Singh et al., 2004; Valko et al., 2007).

Since the pre-historic era, plants, and other living organisms are used by primitive societies due to healing properties, being sought through botanical preparations. In fact, natural matrices, in particular, plants are extremely rich sources of natural biomolecules. Despite, with the passage of time, their use, as prevention or treatment of various conditions, became secondary and recently, plants have deserved a great relevance for scientific researchers that have been studying their bioactive properties; many of those properties have been related to their richness in phenolic compounds (Carocho and Ferreira, 2013a; Murray and Pizzorno, 1998; Wojdylo et al., 2007). Therefore, in the present manuscript, the activity of phenolic extracts and compounds against Candida species was revised and highlighted as an alternative to current antifungal drugs, which failed in treatment of several opportunistic fungal infections related with Candida species.

\section{Opportunistic fungal infections}

\subsection{Historical perspective}

Microorganisms are ubiquitous in the world, with a wide and fascinating variety and diversity. In the absence of microorganisms, the life on earth is not possible, with a close dependency on them. Otherwise, they are responsible for the onset of several diseases and disorders in the host (Amara and Shibl, 2013; Isolauri et al., 2002; Kaur et al., 2009).

In particular, yeasts, which belong to a category of fungi are part of the commensal flora of the healthy population. Colonization by some yeast species is beneficial to the host, because it not only limits the growth of other opportunistic pathogenic fungi, but also stimulates the functioning of the immune system. However, in face to an abnormal overgrowth, they are able to cause a wide variety of dysfunctions/disorders to the host. Candida species are a good example; although they are deemed a commensal microorganism, living smoothly in the inner warm creases and crevices of the gastrointestinal (GI) tract (and vaginal tract), they are able to cause problems, mainly vaginal infections (Asmundsdóttir et al., 2009; Brunke and Hube, 2013; Eggimann et al., 2003a; McCullough et al., 1996; Tsai et al., 2013).

During some days after birth, primarily, the mucosa of GI tract and upper respiratory passages are colonized by those species, as well as mouth, pharynx and larynx. Apart of the observed biochemical variations in the last trimester of pregnancy, namely progesterone, estradiol and glycogen levels, associated with an increase in vaginal $\mathrm{pH}$, which favors the emergence of these infections (Carrara et al., 2010; Vázquez-González et al., 2013), other clinical status increase the vulnerability to yeast infections (diabetes, patient's submitted to broad-spectrum of antibiotic therapy, the use of oral contraceptives) (Epstein and Polsky, 1998; Geiger et al., 1995; Liu et al., 2009; Lott et al., 2005; Sobel, 2007; Tarry et al., 2005). Furthermore, and not least important, the increasing magnitude of organ transplantations, the rise of AIDS and the use of invasive devices (catheters, artificial joints and valves) are also major factors that contribute to higher patient's susceptibility to infections in hospitals (Epstein and Polsky, 1998; Kim and Sudbery, 2011; Pierce, 2005; Vázquez-González et al., 2013).

Seven Candida species are classified as having major medical importance, namely Candida albicans Candida tropicalis, Candida glabrata, Candida parapsilosis, Candida stellatoidea, Candida krusei and Candida kyfer, being Candida albicans the most important (Greenberg and Glick, 2003; McCullough et al., 1996; Silva et al., 2011a; Sullivan et al., 2004; Westwater et al., 2007).

Candidiasis, the most common opportunistic yeast infection in the world has been related in majority with Candida albicans. This microorganism is a causative agent of mouth and mucocutaneous infections, among others more complicated, such as septicemia, endocarditis, meningitis, and peritonitis, especially in patients with reduced immune function or taking antibiotic therapies (Brunke and Hube, 2013; Greenberg and Glick, 2003; McCullough et al., 1996). Nevertheless, in the last two decades, in face to the rapid increase in the incidence of opportunistic fungal infections, numerous studies had revealed that also other species of 
fungi are involved in some hospital infections, like Aspergillus spp., Zygomyces spp., Fusarium spp., Scedosporium spp., Cryptococcus spp., Trichosporon spp., Geotrichum spp., and Rhodotorula spp. (Kwamin et al., 2013; Martins et al., 2014; Tsai et al., 2013; Vázquez-González et al., 2013). But, C. albicans still counting nearly 50-90\% of isolates from fungal infections, being able to cause serious hematogenous infections that, directly, affect the welfare of individuals (VázquezGonzález et al., 2013).

\subsection{Anti-Candida targets}

Due to the high incidence of opportunistic fungal infections, namely triggered by Candida species, a wide variety of experimental studies have been carried out towards the discovery of effective treatments against these opportunistic and pathogenic microorganisms. Cellular morphology, physiology, and metabolism are crucial aspects in fungal cells survival.

All living organisms communicate with the surrounding environment and, in the case of Candida cells, the communication is established through the cellular membrane. Quorum-sensing is considered the mechanism of microbial communication, and for $C$. albicans, the main quorum sensing molecules are farnesol, tyrosol and dodecanol. This mechanism is not only incited by yeast growth but also by the hyphal formation (Lu et al., 2006; Mayer et al., 2013; Wyk et al., 2009). One of the main functions of cellular membrane is to maintain cells homeostasis to ensure the protection of internal organelles, being the first barrier in Candida cells. In the membrane, there are a wide variety of components present, playing different functions. The phospholipid bilayer is a common membrane to all eukaryotic cells, as also proteins, carbohydrates, other lipids (i.e. sterols, fatty acids, glycerol and esters) and even association of those substances. In the case of fungal cells, such as Candida species, ergosterol is the specific and most abundant sterol. Those molecules play important roles in the maintenance of integrity and fluidity of the membrane, acting as secondary messenger in the signal development in order to warrant the proper cell function (i.e. growth, division and reproduction). Thus, ergosterol is considered the main target of antifungal drugs (Ghannoum and Rice, 1999; Kanafani and Perfect, 2008; White et al., 1998).

Chemical substances could act by three main different ways in Candida cells: in the ergosterol biosynthesis (i.e. azoles) (Ghannoum and Rice, 1999; Kanafani and Perfect, 2008; Lamb et al., 1999; Lupetti et al., 2002; Sanglard, 2002); interacting with ergosterol and intercalating between membrane (i.e. polyenes) (Ghannoum and Rice, 1999; Kanafani and Perfect, 2008; Kontoyiannis and Lewis, 2002); or even causing ergosterol depletion (i.e. allylamides) (Kanafani and Perfect, 2008; Kontoyiannis and Lewis, 2002; White et al., 1998).

Unfortunately, in the last years, it has been observed an increasing drug resistance to those classes of antifungal agents, being ineffective even in higher doses. Some virulence mechanisms have been studied, not only to explain the increasing rates of antifungal drugs resistance, but also to discover/create new and promising antifungal strategies. So far, the most frequent virulence mechanism associated with Candida species infections is the ability of adaptation to a wide variety of habitats, commonly known as morphological transition. Notwithstanding, other factors, as the ability for biofilm formation, the secretion of hydrolytic enzymes (such as proteases and phospholipases), and the expression of adhesins and invasins linked with the dimorphic phases and phenotypic switching, have been associated with Candida species infections (Mayer et al., 2013; Tsai et al., 2013).

In this sense, different molecular targets needs to be studied as also more effective and selective compounds against the fungal cell wall; compounds to inhibit nucleic acids, to affect the plasma membrane, ergosterol, phospholipid, sphingolipid and pro- tein biosynthesis; intermediates of the metabolism of nucleic acids and amino acids; compounds that affect efflux pumps, proton ATPases and other enzymes. The study of the fungal virulence factors and involved genes on antifungal resistance, among other important biological components with a direct interference on drug resistance, is crucial (Franz et al., 1998; Kanafani and Perfect, 2008; Lupetti et al., 2003; Mayer et al., 2013; Noël, 2012; Perlin, 2014; Sangamwar et al., 2008; Sanglard and Odds, 2002; White et al., 2002, 1998). Mayer et al. (2013) quoted polymorphism, secretion of hydrolases; $\mathrm{pH}$ sensing and regulation; metabolic adaptation; environmental stress response; heat shock proteins and small heat shock proteins, and metal acquisition as the most important virulence factors and fitness attributes, while Lewis et al. (2012) proposed that the inhibition of macrophage cell division or arrest of the host macrophages to confine the pathogens is another virulence attribute of Candida species, in particular C. albicans.

\subsection{Current anti-Candida drugs}

Currently, there are five main classes of antifungal agents widely used at a medical level against the major fungal pathogens, including Candida species: polyenes, azoles, allylamines/thiocarbamates, fluoropyramidines and echinocandins (Maurya et al., 2013).

Azoles, polyenes and allylamine/thiocarbamates act, direct or indirectly, in the ergosterol present in all Candida species (Kontoyiannis and Lewis, 2002; Lupetti et al., 2003).

Polyenes comprise a class of antifungal agents that affect cellular membranes containing ergosterol. These agents present amphipathic properties which allow their intercalation between membranes containing ergosterol, forming channels that favour the ionic unbalance and destroy the proton gradient (Ghannoum and Rice, 1999). Nystatin and amphothericin-B are the most common used polyenes (Eggimann et al., 2003b). Nevertheless, not only primary but also secondary resistance of Candida species, namely C. albicans, C. lusitanea, C. guilliermondii and C. glabrata, have been observed (Ghannoum and Rice, 1999; Kontoyiannis and Lewis, 2002; White et al., 1998).

Azoles are another class of antifungal agents in which the target is over again ergosterol, but in this case affects its biosynthesis. Firstly introduced during the 1980s and used by oral administration, to treat oropharyngeal candidiasis in patients with $\mathrm{VIH}^{+}$, they act as inhibitors of sterol $14 \alpha$-demethylation. The remotion of the C32-methyl group at a $14 \alpha$ - from precursor sterols, catalyzed by a microsomal cytochrome P450 monooxygenase, affect the main sterol present on fungal cells membranes: Erg11p, a lanosterol 14alpha-demethylase. Miconazole and econazole were the original used azole compounds, followed by ketoconazole, fluconazole and itraconazole (Eggimann et al., 2003b; Ghannoum and Rice, 1999; Sardi et al., 2013). However, and despite during several years those drugs presented a great efficacy as antifungal agents, resulting in a widespread use, currently it is being observed an increasing emergence of Candida species-resistance (namely C. albicans, C. glabrata, C. parapsilosis, C. krusei and C. lusitaniae) (Develoux and Bretagne, 2005; Noël, 2012; Vázquez-González et al., 2013).

Allylamines (i.e. naftifine and terbinafine) and thiocarbamates (i.e. tolnaftate and tolciclate) are the third class of antifungal agents that interact with ergosterol. But, in this case, they act as inhibitors of squalene epoxidase, blocking the conversion of squalene into 2,3-oxidosqualene, that result in ergosterol depletion and accumulation of toxic sterols. It was shown that the increasing of the squalene levels is toxic, leading to cell membrane permeability and then disrupting the cellular organization (Ghannoum and Rice, 1999; White et al., 1998). However, this class of antifungal drugs presents a weak activity against the most frequent Candida species, being only active against dermatophytes (Kontoyiannis and Lewis, 2002; Sanglard, 2002). 
On the other hand, fluoropyramidines and echinocandins act differently from the previous drugs; the first act as inhibitor of nucleic acids, while the second as inhibitor of the glucan synthesis and, therefore, are effective against Candida biofilm cells (Kontoyiannis and Lewis, 2002; Pereira Gonzales and Maisch, 2012). Fluoropyrimidines, namely 5-fluorocytosine (5FC) is a fluorinated pyrimidine that impairs nucleic acid biosynthesis due to the formation of toxic fluorinated pyrimidine antimetabolites. A permease enzyme aids $5 F C$ to enter on fungal cells, where it is converted into 5 -fluorouracil ( $5 \mathrm{FU}$ ), catalyzed by the cytosine deaminase. After this, 5FU is converted into 5-fluorouridylic acid (FUMP) and, then, phosphorylated and incorporated into RNA, leading to a disruption of protein synthesis. Another mechanism of action of 5FU, results of its conversion into 5-fluorodeoxyuridine monophosphate, a potent inhibitor of the enzyme involved in DNA synthesis and nuclear division (Waldorf and Polak, 1983). There are some studies that refer an increasing resistance to 5FC (Ghannoum and Rice, 1999; Gubbins and Anaissie, 2009; Liu et al., 2009; Souza et al., 2010), and despite several mechanisms of action responsible for 5FC resistance are still not established, they could be related with losses of permease activity or decrease of enzymatic activity responsible for the conversion of 5FC into FMUP. This process was observed in C. glabrata, while mutational losses of one of the pyrimidine salvage enzymes and decrease in UPRTase in a gene dose-dependent manner were observed in C. albicans strains, resistant to 5FC (Ghannoum and Rice, 1999).

Lastly, echinocandins (i.e. caspofungin, anidulofungin, and micafungin) are the latest and, at the same time, the first new class of antifungal drugs. The main target of this drug is the fungal cell wall, acting as inhibitors of the $\alpha-1,3-\mathrm{D}$-glucan biosynthesis by blocking of $\beta-1,3-D-$ glucan synthase (Perlin, 2014; Sanglard and Odds, 2002). Despite a higher level of efficiency associated to this class of antifungal drugs, exerting rapid fungicidal effects against Candida species, including azole-resistance species (Kontoyiannis and Lewis, 2002), in the last years, it has been observed an increasing level of resistance, namely for C. albicans, C. glabrata, C. krusei and C. parapsilosis (Kanafani and Perfect, 2008; Perlin, 2009). The involved resistance mechanisms were not completely elucidated and other are still unknown; however, drug tolerance conferred by adaptive cellular physiology to the environmental stress, as well as genetic mutations and amino acids substitutions are the main supposed contributors associated with Candida species echinocandin-resistant (Perlin, 2009).

Nevertheless, new perspectives of development of new orally effective drugs are open and different experiments have been made (Kanafani and Perfect, 2008; Perlin, 2014, 2009; Sanglard, 2002). So, considering all the advances on antifungal drugs research, it is necessary to take into account the safety, tolerability, efficacy, toxicity, and even side effects of these substances/chemical molecules. In this sense, other alternatives safer than the current antifungal drugs are necessary.

\section{Active principles of plants}

\subsection{Plants as a rich source of bioactive compounds}

Plants have been used for several generations in traditional herbal medicine, as first choice treatment. The first civilizations established the therapeutic potential of plants taking into account the more or less prominent effects observed in the body. They also observed that certain plants evidenced toxic effects according to the dose. However, they did not know the reasons that provided those effects, as well as, the nature of plant constituents. Thus, much of the knowledge was empirical, passing from generation to generation, but lacking of a solid foundation of the use and pre- scription, mostly due to the low literacy and written information (Halberstein, 2005; Murray and Pizzorno, 1998; Petrovska, 2012).

Currently, it is well recognized that hundreds of biological active chemical compounds are present, in each plant, working in synergism, and conferring a broad variety of bioactivities, being subject of an increasing scientific interest. More and more, people worldwide use medicinal plants as first priority to maintain a good health, to prevent or even treat some health conditions. Linked to this, some studies have been conducted, using a wide variety of plants, towards the identification of bioactive molecules, their properties and mechanisms of action (Agarwal et al., 2010; Alves-Silva et al., 2013; Asgarpanah and Kazemivash, 2012; Asl and Hosseinzadeh, 2008; Bakkali et al., 2008; Kanafani and Perfect, 2008; Rana et al., 2011; Sher, 2009; Shojaii and Fard, 2012; Silva et al., 2011b; Singh et al., 2010). In fact, natural matrices, in particular, plants are extremely rich sources of biomolecules that have sparked an increasing interest for scientific researchers, in different areas of knowledge. Until now, several chemical compounds have been identified, but only a few properties have been clarified, with many remaining undefined. Nevertheless, many compounds are still unknown, as also the synergic interactions among them (Carocho and Ferreira, 2013a; Choudhary and Atta-ur-Rahmant, 1999; Devasagayam et al., 2004; Rubió et al., 2013).

It is truly believed that the biologically active chemical compounds present in plants act synergistically and confer, both direct and indirect effects, to the human body. The direct impact is commonly due to their phytopharmacological action, while the indirect impact is linked to the simultaneous interaction with other plants or drugs. Furthermore, it should be highlighted that the fact that one plant from a particular genus contains a specific chemical composition and a significant medicinal value does not mean that all the other plants from the same genus had the same value. Even some studies report that the same species, but with different origins might have considerable differences on chemical composition and, therefore, a different efficacy and potency in the bioactivity (Farhat et al., 2009, 2013; Ghasemi Pirbalouti et al., 2013; Papageorgiou et al., 2008; Politeo et al., 2006).

In the modern medical classification, a plant acquire the "medicinal" status when it presents several pharmacologically active compounds, making possible its use at a therapeutic level, conferring not only benefits in the treatment of some conditions, but also in prevention/prophylaxis (Balch, 2006; Murray, 2004; Murray and Pizzorno, 1998). Notwithstanding, other chemical compounds present in trace amounts could influence the action of the main molecules, which explains the higher potential of using the whole natural matrix instead of an isolate chemical compound (Ettefagh et al., 2011; Junio et al., 2011).

Thus, it is very important to provide a correct and complete characterization of the bioactive compounds present in medicinal plants, in order to complement and unify the prescriptions, and at the same time, to establish the amount/dosage of active principles needed to treat/avert various health problems. Currently, due to the advances of the analytical methods, it was possible to elucidate the structure of thousands of phytochemicals, either primary or secondary metabolites (Fig. 1).

\subsection{Primary metabolites}

Primary metabolites comprise the organic compounds derived from the primary metabolism of plants; they are often concentrated in seeds and/or vegetative organs and play an important role in the physiological development and basic cell metabolism. In general, these compounds are not limited to the plant kingdom, being even used in several biosynthetic pathways (Nelson and Cox, 2000).

Higher plants are able to synthetize organic compounds and produce oxygen from the light, water and carbon dioxide, which 


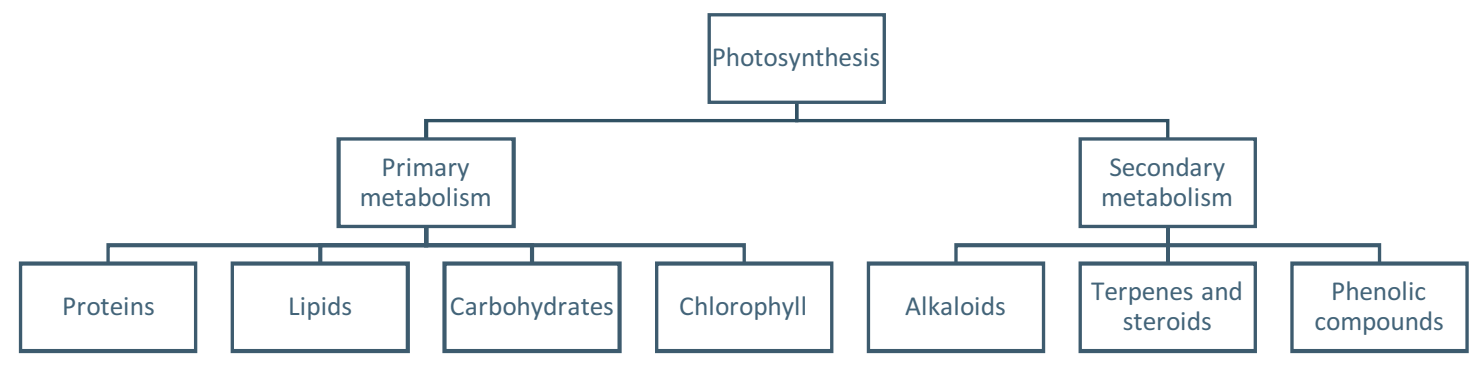

Fig. 1. Relationship between the primary and secondary metabolism in plants.

are the main energetic and food sources for animals. Furthermore, these same organic compounds are also an energetic source for biosynthetic organisms. This process, in which autotrophic organisms produce organic compounds according to their needs is commonly known as photosynthesis: light phase and dark phase. Organic compounds are synthetized in the dark phase, through some biosynthetic pathways (Nelson and Cox, 2000). Proteins, lipids, carbohydrates and chlorophyll are the main compounds derived from the primary metabolism (Fig. 1).

Besides many other physiological functions, proteins and even some free amino acids are considered crucial transport elements of a wide variety of molecules; integrate the genetic material binding to nucleic acids; include enzymes play an important reserve role and confer high protection against invaders (Nelson and Cox, 2000; Proença da Cunha et al., 2010, 2012). On the other hand, lipids, commonly defined as water insoluble compounds but soluble in organic solvents play important organic benefits as structural components of cellular membranes; energetic reserve; protective function; hormonal regulators and synthesizers of inflammatory immunomodulators (eicosanoids: prostaglandins, thromboxanes and leukotrienes), among other crucial functions in living organisms (Campos, 2008; Nelson and Cox, 2000).

Carbohydrates, or glycids, represent the basis of dietary food of a majority of living organisms. According to the length and the type of glycosidic linkages, these molecules play different biological properties and have innumerous applications. One of the most common is their energetic cellular reservoir function, being accumulated in the main reserve organs of plants - seeds, grains and tubers, as well as leaves. The latest have a limited storage capacity (Campos, 2008; Nelson and Cox, 2000; Proença da Cunha et al., 2010).

Lastly, chlorophyll, a green pigment mainly present in the higher plant protoplasts, but also in red algae and purple bacteria, essentially exerts functions of photosynthetic pigments. Despite the existence of two types of chlorophyll, $a$ and $b$, both of them are involved in organic compounds synthesis and, therefore, in the primary function of plants: nutrition (Campos, 2008; Nelson and Cox, 2000).

In fact, primary metabolites are much more than merely macronutrients. Some of the bioactive properties reported for plants, such as antioxidant, antimicrobial, anti-inflammatory, and antitumor activities have been related with primary metabolites, mainly lipids (polyunsaturated fatty acids, sterols), proteins, carotenoids, vitamins and carbohydrates (polysaccharides and glycosides) (Ksouri et al., 2012).

\subsection{Secondary metabolites}

Secondary metabolites are organic compounds that derived from the primary metabolites of plants. It is well established that these metabolites play specialized functions in a wide variety of living organisms, being even considered as biologically active compounds. They are renowned as possess higher value than the primary metabolites, in relation to the life expectancy, wellbeing and even to prevent/avert some diseases/disorders (Nelson and Cox, 2000; Rubió et al., 2013).

Terpenes are a class of secondary metabolites formed by several isoprene units, i.e. several combinations of 5-carbon-base (C5) Different combinations of the isoprene units originate structurally and functionally different classes of terpenes (Rubió et al., 2013) Thus, they could occur as monoterpenes (C10), diterpenes (C20), triterpenes (C30), tetraterpenes (C40), but also hemiterpenes (C5) and sesquiterpenes (C15). Monoterpenes and diterpenes are the main classes of terpenes present on herbs and spices. Furthermore, these compounds have important biological activities, including antimicrobial properties against a wide variety of pathogens such as Candida species (Alves et al., 2013; Rubió et al., 2013; Saleem et al., 2010).

Steroids are secondary metabolites derived from the tetracyclic triterpenes (C30), composed by isoprenoid C5 units derived from isopentenyl (3-methylbut-3-en-L-yL) pyrophosphate, and presenting a characteristic branched chain structure. Stigmasterol has been reported as one of the most abundant steroids in plant oils. These compounds are commonly highly degraded into other products, namely steroid hormones that play important organic functions (Carocho and Ferreira, 2013b; Nelson and Cox, 2000). Moreover, the antimicrobial activity of steroids has also been described, including the antifungal properties against some pathogens such as Candida species (Alves et al., 2013; Saleem et al., 2010; Sanglard, 2002). One of the proposed mechanisms of action is their ability to disrupt membrane integrity (Saleem et al., 2010), but others remain unclear and even unknown.

Alkaloids comprise a diverse group of heterocyclic nitrogen substances, being commonly considered high toxic molecules that play an important defensive role, but reflecting a general biological principle of poisonous plants (Inbaraj et al., 2001, 2006; Sertel et al., 2011). Nevertheless, in some situations, their toxicity is benefic; for example, Sertel et al. (2011) reported that vinblastine, vincristine (from Catharanthus roseus, formerly: Vinca rosea) and semisynthetic vindesine and vinorelbine, presented high cytotoxicity against cancer cells, highlighting the potential of these dimeric indole alkaloids for cancer, leukemia and lymphomas treatments. The efficacy of alkaloids against bacteria, parasites, dermatophytes and fungi, including Candida species has also been described (AliShtayeh and Ghdeib, 1999; Arif et al., 2011, 2009; Carocho and Ferreira, 2013b; Dzoyem et al., 2014; Rose, 1999; Sher, 2009; Silva and Fernandes Júnior, 2010; Wyk et al., 2009; Xie et al., 2012). The intercalation into cell wall and/or DNA is considered one of the main described mechanisms of bioactive action of alkaloids (Silva and Fernandes Júnior, 2010).

Phenolic compounds constitute a major class of plant secondary metabolites being broadly distributed, with more than 8000 phenolic structures currently identified. These compounds can contain one or more aromatic rings with one or more hydroxyl groups (Dai and Mumper, 2010). In fact, the term "phenolic" or "polyphenol" can be chemically defined as a substance which possesses an aromatic ring bearing at least one (phenol) or more (polyphe- 


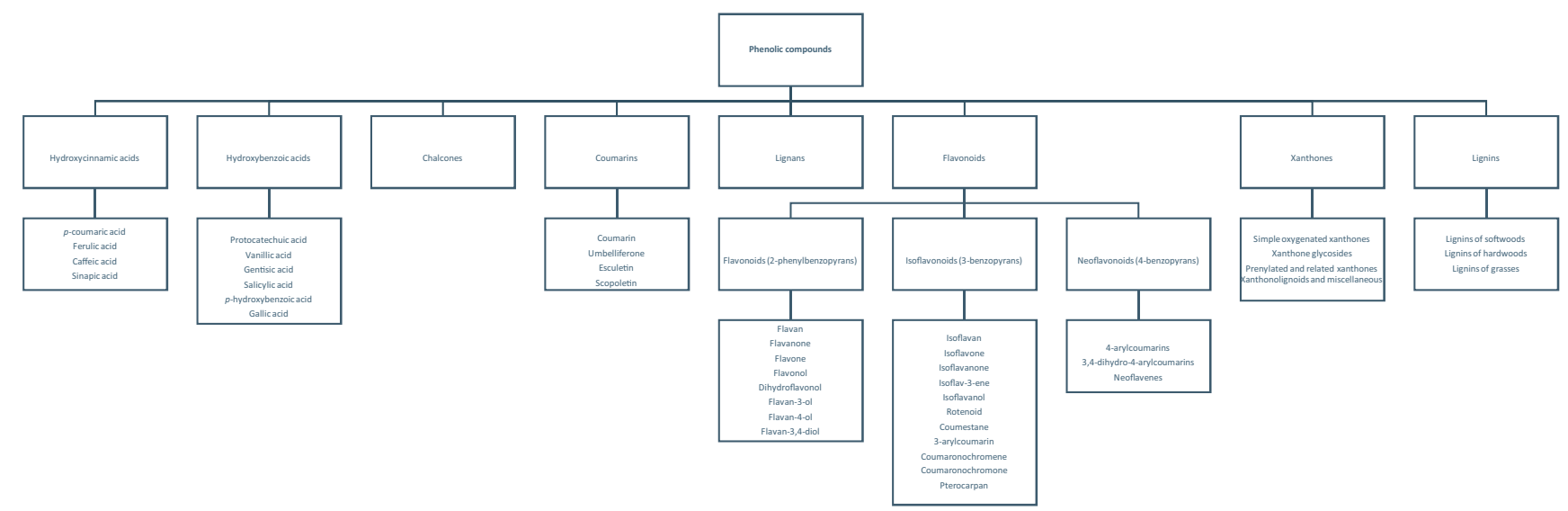

Fig. 2. Main classes and subclasses of phenolic compounds.

nol) hydroxyl substituent, including functional derivatives (esters, methyl ethers, glycosides, etc.), ranging from simple molecules, such as phenolic acids, to high complex structures, such as tannins (Dai and Mumper, 2010; Santos-Buelga et al., 2012). In the modern classification, the broad category of phenolics is divided into several groups, according to the number of phenol subunits present: polyphenols and simple phenols. Thus, polyphenols possessing at least two phenol subunits are classified as flavonoids, while those compounds which possess three or more phenol subunits are included in tannins (hydrolysable and non-hydrolysable) (Giada, 2013; Robbins, 2003). Phenolic acids, flavonoids and tannins are the most common, being stilbenes and lignans less common (Dai and Mumper, 2010). Curiously, these classes of phenolic compounds are currently the main subjects of scientific researches. But, beyond these, other phenolic compounds, like chalcones, coumarins, xanthones and lignins are also present in natural matrices (Fig. 2).

Several researchers have demonstrated the antioxidant potential of phenolic compounds (Çekiç et al., 2013; Rice-Evans et al., 1996; Walch et al., 2011), but currently other bioactive properties have also been studied. Curiously, some of these biological properties are directly linked to the antioxidant activity. It is a fact, that the chemical structure of phenolic compounds determine their properties as well as mechanism of action, being conferred by different ways (Giada, 2013). Nevertheless, and not less important is the fact that despite reactive oxygen and nitrogen species (ROS/RNS) at physiological concentrations may be required for normal cell functioning and to play an important role in plant and animal evolutions, these species are able to attack biological macromolecules (e.g. cellular DNA), causing a wide variety of cellular damages, disorders and even diseases; to disestablish of the defense systems increasing the vulnerability to infections by pathogens, parasites and other invaders (Carocho and Ferreira, 2013a; Çekiç et al., 2013; Chaturvedi and Beal, 2013; Devasagayam et al., 2004; Valko et al., 2007).

\section{Activity of phenolic extracts/compounds against Candida species}

\subsection{The use of phenolic extracts against Candida species}

Several studies have been performed in order to evaluate the antifungal potential of polar extracts from plant origin, which are enriched in phenolic compounds, such as acetone (Table 1), aqueous (Table 2), ethanolic (Table 3), methanol-dichloromethane (Table 4), methanolic (Table 5), hydroalcoholic (methanol:or ethanol:water mixtures) (Table 6), DMSO, diethyl ether and ethyl acetate extracts, and tinctures (Table 7). The aqueous extracts were the most studied preparations, followed by methanolic and ethanolic extracts.

Different techniques/assays have been used for that purpose. Agar disc diffusion and broth dilution (microdilution and macrodilution) methods are the most commonly used, being, normally, performed according to the Clinical and Laboratory Standards Institute (CLSI), with some modifications. Both of them are considered antimicrobial susceptibility tests, expressing the results as halo inhibition zones or minimal inhibitory (MIC) and minimal fungicidal (MFC) concentrations, respectively. The most tested Candida species were C. albicans, C. tropicalis, C. krusei, C. glabrata, C. parapsilosis, $C$. lusitaniae and $C$. guilliermondii, being considered the main species associated to opportunistic fungal infections (VázquezGonzález et al., 2013).

In general, the plant extracts obtained using methanol and water, as solvent extraction, and mixtures of the previous (hydroalcoholic extracts) were more effective against Candida species than the other extracts prepared with a unique solvent. Thus, the methanol (Table 5), aqueous (Table 2) and hydroalcoholic extracts (ethanol:water and methanol:water mixtures) (Table 6) presented lower MICs than the extracts obtained with acetone (Table 1), methanol-dichloromethane (Table 4) ethanol (Table 3) or other

Table 1

Activity of acetone extracts against Candida species.

\begin{tabular}{|c|c|c|c|}
\hline Plant source & Candida species & MIC & References \\
\hline Combretum albopunctatum & C. albicans & $0.64 \mathrm{mg} / \mathrm{mL}$ & Masoko et al. (2010) \\
\hline Combretum imberbe & C. albicans & $2.5 \mathrm{mg} / \mathrm{mL}$ & Masoko et al. (2010) \\
\hline Combretum nelsonii & C. albicans & $0.04 \mathrm{mg} / \mathrm{mL}$ & Masoko et al. (2010) \\
\hline Origanum vulgare & C. albicans & $5 \mathrm{mg} / \mathrm{mL}$ & Ličina et al. (2013) \\
\hline Paullinia cupana & C. albicans & $250 \mu \mathrm{m} / \mathrm{mL}$ & Basile et al. (2013) \\
\hline Sclerocarya birrea & C. parapsilosis & $0.32 \mathrm{mg} / \mathrm{mL}$ & Masoko et al. (2008) \\
\hline Terminalia sericea & C. albicans & $0.64 \mathrm{mg} / \mathrm{mL}$ & Masoko et al. (2010) \\
\hline
\end{tabular}

MIC - minimum inhibitory concentration. 
Table 2

Activity of aqueous extracts against Candida species.

\begin{tabular}{|c|c|c|c|}
\hline Plant source & Candida species & MIC & References \\
\hline \multirow[t]{4}{*}{ Acorus calamus } & C. albicans & $12.5 \mathrm{mg} / \mathrm{mL}$ & \multirow[t]{4}{*}{ Webster et al. (2008) } \\
\hline & C. krusei & $12.5 \mathrm{mg} / \mathrm{mL}$ & \\
\hline & C. lusitaniae & $12.5 \mathrm{mg} / \mathrm{mL}$ & \\
\hline & C. parapsilosis & $12.5 \mathrm{mg} / \mathrm{mL}$ & \\
\hline Adiantum capillus veneris & C. albicans & $1 \mathrm{mg} / \mathrm{mL}^{\mathrm{a}}$ & Ishaq et al. (2014) \\
\hline \multirow[t]{11}{*}{ Allium sativum } & \multirow[t]{3}{*}{ C. albicans } & $0.2 \mathrm{mg} / \mathrm{mL}$ & Shamim et al. (2004) \\
\hline & & $100 \mathrm{mg} / \mathrm{mL}^{\mathrm{a}}$ & Fei et al. (2008) \\
\hline & & $1 \mathrm{mg} / \mathrm{mL}^{\mathrm{a}}$ & Pathak (2012) \\
\hline & C. dublinensis & $1 \mathrm{mg} / \mathrm{mL}^{\mathrm{a}}$ & Pathak (2012) \\
\hline & \multirow[t]{2}{*}{ C. grabrata } & $0.2 \mathrm{mg} / \mathrm{mL}$ & Shamim et al. (2004) \\
\hline & & $1 \mathrm{mg} / \mathrm{mL}^{\mathrm{a}}$ & Pathak (2012) \\
\hline & \multirow[t]{2}{*}{ C. krusei } & $100 \mathrm{mg} / \mathrm{mL}^{\mathrm{a}}$ & Fei et al. (2008) \\
\hline & & $1 \mathrm{mg} / \mathrm{mL}^{\mathrm{a}}$ & Pathak (2012) \\
\hline & \multirow[t]{3}{*}{ C. tropicalis } & $0.2 \mathrm{mg} / \mathrm{mL}$ & Shamim et al. (2004) \\
\hline & & $100 \mathrm{mg} / \mathrm{mL}^{\mathrm{a}}$ & Fei et al. (2008) \\
\hline & & $1 \mathrm{mg} / \mathrm{mL}^{\mathrm{a}}$ & Pathak (2012) \\
\hline \multirow[t]{6}{*}{ Alnus viridis } & C. albicans & $12.5 \mathrm{mg} / \mathrm{mL}$ & \multirow[t]{6}{*}{ Webster et al. (2008) } \\
\hline & C. glabrata & $25 \mathrm{mg} / \mathrm{mL}$ & \\
\hline & C. krusei & $0.2 \mathrm{mg} / \mathrm{mL}$ & \\
\hline & C. lusitaniae & $6 \mathrm{mg} / \mathrm{mL}$ & \\
\hline & C. parapsilosis & $25 \mathrm{mg} / \mathrm{mL}$ & \\
\hline & C. tropicalis & $3 \mathrm{mg} / \mathrm{mL}$ & \\
\hline \multirow[t]{3}{*}{ Aloe barbadensis } & C. albicans & $1 \mathrm{mg} / \mathrm{mL}$ & \multirow[t]{3}{*}{ Shamim et al. (2004) } \\
\hline & C. grabrata & $1 \mathrm{mg} / \mathrm{mL}$ & \\
\hline & C. tropicalis & $0.2 \mathrm{mg} / \mathrm{mL}$ & \\
\hline Aloe excelsa & C. albicans & $1 \mathrm{mg} / \mathrm{mL}$ & Coopoosamy and Magwa (2007) \\
\hline & C. tropicalis & $0.2 \mathrm{mg} / \mathrm{mL}$ & \\
\hline Arbutus unedo & C. albicans & $10 \mathrm{mg} / \mathrm{mL}^{\mathrm{a}}$ & Dib et al. (2010) \\
\hline Arctium minus & C. albicans & $12.5 \mathrm{mg} / \mathrm{mL}$ & Lubian et al. (2010) \\
\hline & C. dubliniensis & $12.5 \mathrm{mg} / \mathrm{mL}$ & \\
\hline & C. glabrata & $12.5 \mathrm{mg} / \mathrm{mL}$ & \\
\hline & C. krusei & $12.5 \mathrm{mg} / \mathrm{mL}$ & \\
\hline & C. stellatoideia & $12.5 \mathrm{mg} / \mathrm{mL}$ & \\
\hline & C. tropicalis & $25 \mathrm{mg} / \mathrm{mL}$ & \\
\hline & & $12.5 \mathrm{mg} / \mathrm{mL}$ & \\
\hline Artemisia campestris & C. glabrata & $25 \mathrm{mg} / \mathrm{mL}$ & Webster et al. (2008) \\
\hline & C. krusei & $50 \mathrm{mg} / \mathrm{mL}$ & \\
\hline & C. lusitaniae & $25 \mathrm{mg} / \mathrm{mL}$ & \\
\hline & C. parapsilosis & $50 \mathrm{mg} / \mathrm{mL}$ & \\
\hline & C. tropicalis & $25 \mathrm{mg} / \mathrm{mL}$ & \\
\hline Artemisia frigida & C. glabrata & $0.4 \mathrm{mg} / \mathrm{mL}$ & Webster et al. (2008) \\
\hline & C. krusei & $6 \mathrm{mg} / \mathrm{mL}$ & \\
\hline & C. lusitaniae & $12.5 \mathrm{mg} / \mathrm{mL}$ & \\
\hline & C. parapsilosis & $50 \mathrm{mg} / \mathrm{mL}$ & \\
\hline & C. tropicalis & $6 \mathrm{mg} / \mathrm{mL}$ & \\
\hline Azadirachta indica & C. albicans & $1 \mathrm{mg} / \mathrm{mL}^{\mathrm{a}}$ & Pathak (2012) \\
\hline & C. dublinensis & $1 \mathrm{mg} / \mathrm{mL}^{\mathrm{a}}$ & \\
\hline & C. glabrata & $1 \mathrm{mg} / \mathrm{mL}^{\mathrm{a}}$ & \\
\hline & C. krusei & $1 \mathrm{mg} / \mathrm{mL}^{\mathrm{a}}$ & \\
\hline & C. tropicalis & $1 \mathrm{mg} / \mathrm{mL}^{\mathrm{a}}$ & \\
\hline Betula alleghaniensis & C. albicans & $0.8 \mathrm{mg} / \mathrm{mL}$ & Webster et al. (2008) \\
\hline & C. glabrata & $0.05 \mathrm{mg} / \mathrm{mL}$ & \\
\hline & C. krusei & $0.4 \mathrm{mg} / \mathrm{mL}$ & \\
\hline & C. lusitaniae & $0.4 \mathrm{mg} / \mathrm{mL}$ & \\
\hline & C. parapsilosis & $3 \mathrm{mg} / \mathrm{mL}$ & \\
\hline & & $0.05 \mathrm{mg} / \mathrm{mL}$ & \\
\hline & C. tropicalis & $6 \mathrm{mg} / \mathrm{mL}$ & \\
\hline Brucea javanica & C. albicans & $50 \mathrm{mg} / \mathrm{mL}$ & Nordin et al. (2013) \\
\hline & C. dubliniensis & $3.13 \mathrm{mg} / \mathrm{mL}$ & \\
\hline & C. glabrata & $25 \mathrm{mg} / \mathrm{mL}$ & \\
\hline & C. krusei & $50 \mathrm{mg} / \mathrm{mL}$ & \\
\hline & C. lusitaniae & $25 \mathrm{mg} / \mathrm{mL}$ & \\
\hline & C. parapsilosis & $25 \mathrm{mg} / \mathrm{mL}$ & \\
\hline & C. tropicalis & $50 \mathrm{mg} / \mathrm{mL}$ & \\
\hline Cassia alata & C. albicans & $26.90 \mathrm{mg}$ & Timothy et al. (2012) \\
\hline Camellia sinensis & C. albicans & $10 \mathrm{mg} / \mathrm{mL}^{\mathrm{a}}$ & Doddanna et al. (2013) \\
\hline Cinnamomum sp. & C. albicanus & $200 \mathrm{mg} / \mathrm{mL}^{\mathrm{a}}$ & Qader et al. (2013) \\
\hline & C. dublicans & $200 \mathrm{mg} / \mathrm{mL}^{\mathrm{a}}$ & \\
\hline & C. glabrata & $200 \mathrm{mg} / \mathrm{mL}^{\mathrm{a}}$ & \\
\hline & C. krusei & $200 \mathrm{mg} / \mathrm{mL}^{\mathrm{a}}$ & \\
\hline & C. tropicalis & $200 \mathrm{mg} / \mathrm{mL}^{\mathrm{a}}$ & \\
\hline Emblica officinalis & C. albicans & $200 \mathrm{mg} / \mathrm{mL}^{\mathrm{a}}$ & Saeed and Tariq (2007) \\
\hline
\end{tabular}


Table 2 (Continued)

\begin{tabular}{|c|c|c|c|}
\hline Plant source & Candida species & MIC & References \\
\hline \multirow[t]{10}{*}{ Epilobium augustifolium } & \multirow[t]{4}{*}{ C. albicans } & $0.2 \mathrm{mg} / \mathrm{mL}$ & \multirow[t]{10}{*}{ Webster et al. (2008) } \\
\hline & & $0.4 \mathrm{mg} / \mathrm{mL}$ & \\
\hline & & $0.4 \mathrm{mg} / \mathrm{mL}$ & \\
\hline & & $0.4 \mathrm{mg} / \mathrm{mL}$ & \\
\hline & C. glabrata & $0.025 \mathrm{mg} / \mathrm{mL}$ & \\
\hline & C. krusei & $0.2 \mathrm{mg} / \mathrm{mL}$ & \\
\hline & C. lusitaniae & $0.05 \mathrm{mg} / \mathrm{mL}$ & \\
\hline & C. parapsilosis & $0.4 \mathrm{mg} / \mathrm{mL}$ & \\
\hline & & $0.1 \mathrm{mg} / \mathrm{mL}$ & \\
\hline & C. tropicalis & $1.6 \mathrm{mg} / \mathrm{mL}$ & \\
\hline Eucalyptus sideroxylon & C. albicans & $400 \mathrm{mg} / \mathrm{mL}^{\mathrm{a}}$ & Ashour (2008) \\
\hline Eucalyptus torquata & C. albicans & $400 \mathrm{mg} / \mathrm{mL}^{\mathrm{a}}$ & Ashour (2008) \\
\hline \multirow[t]{6}{*}{ Fragaria virginiana } & C. albicans & $0.8 \mathrm{mg} / \mathrm{mL}$ & \multirow[t]{6}{*}{ Webster et al. (2008) } \\
\hline & C. glabrata & $0.05 \mathrm{mg} / \mathrm{mL}$ & \\
\hline & C. krusei & $0.05 \mathrm{mg} / \mathrm{mL}$ & \\
\hline & C. lusitaniae & $0.1 \mathrm{mg} / \mathrm{mL}$ & \\
\hline & C. parapsilosis & $0.2 \mathrm{mg} / \mathrm{mL}$ & \\
\hline & C. tropicalis & $0.8 \mathrm{mg} / \mathrm{mL}$ & \\
\hline Funtumia elastica & C. albicans & $100 \mathrm{mg} / \mathrm{mL}^{\mathrm{a}}$ & Adekunle and Ikumapayi (2006) \\
\hline Hyssopus officinalis subsp. pilifer & C. albicans & $7.00 \mathrm{mg} / \mathrm{mL}$ & Džamić et al. (2013) \\
\hline Lonicera japonica & C. albicans & Viability (\%): 81.48 & Wong and Tsang (2009) \\
\hline Mallotus oppositifolius & C. albicans & $100 \mathrm{mg} / \mathrm{mL}^{\mathrm{a}}$ & Adekunle and Ikumapayi (2006) \\
\hline Morinda citrifolia & C. albicans & $40 \mathrm{mg} / \mathrm{mL}$ & Jainkittivong et al. (2009) \\
\hline Morinda morindoides & C. albicans & $62.50 \mathrm{mg} / \mathrm{mL}$ & Touré et al. (2011) \\
\hline \multirow[t]{5}{*}{ Murraya koenigii } & C. albicans & $1 \mathrm{mg} / \mathrm{mL}^{\mathrm{a}}$ & \multirow[t]{5}{*}{ Pathak (2012) } \\
\hline & C. dublinensis & $1 \mathrm{mg} / \mathrm{mL}^{\mathrm{a}}$ & \\
\hline & C. glabrata & $1 \mathrm{mg} / \mathrm{mL}^{\mathrm{a}}$ & \\
\hline & C. krusei & $1 \mathrm{mg} / \mathrm{mL}^{\mathrm{a}}$ & \\
\hline & C. tropicalis & $1 \mathrm{mg} / \mathrm{mL}^{\mathrm{a}}$ & \\
\hline Ocimum sanctum & C. glabrata & $1 \mathrm{mg} / \mathrm{mL}^{\mathrm{a}}$ & Pathak (2012) \\
\hline \multirow[t]{2}{*}{ Origanum vulgare } & \multirow[t]{2}{*}{ C. albicans } & $200 \mathrm{mg} / \mathrm{mL}^{\mathrm{a}}$ & Abdul et al. (2012) \\
\hline & & $20 \mathrm{mg} / \mathrm{mL}$ & Ličina et al. (2013) \\
\hline Paeonia suffruticosa & C. albicans & Viability (\%): 100.53 & Wong and Tsang (2009) \\
\hline Plantago media & C. albicans & $200 \mathrm{mg} / \mathrm{mL}^{\mathrm{a}}$ & Qader et al. (2013) \\
\hline & C. dublicans & $200 \mathrm{mg} / \mathrm{mL}^{\mathrm{a}}$ & \\
\hline & C. glabrata & $200 \mathrm{mg} / \mathrm{mL}^{\mathrm{a}}$ & \\
\hline & C. krusei & $200 \mathrm{mg} / \mathrm{mL}^{\mathrm{a}}$ & \\
\hline & C. tropicalis & $200 \mathrm{mg} / \mathrm{mL}^{\mathrm{a}}$ & \\
\hline Populus tremuloides & C. albicans & $1.6 \mathrm{mg} / \mathrm{mL}$ & Webster et al. (2008) \\
\hline & C. glabrata & $0.025 \mathrm{mg} / \mathrm{mL}$ & \\
\hline & C. krusei & $0.2 \mathrm{mg} / \mathrm{mL}$ & \\
\hline & C. lusitaniae & $0.2 \mathrm{mg} / \mathrm{mL}$ & \\
\hline & C. parapsilosis & $0.4 \mathrm{mg} / \mathrm{mL}$ & \\
\hline & C. tropicalis & $1.6 \mathrm{mg} / \mathrm{mL}$ & \\
\hline Punica granatum & C. albicans & $200 \mathrm{mg} / \mathrm{mL}^{\mathrm{a}}$ & Qader et al. (2013) \\
\hline & C. dublicans & $200 \mathrm{mg} / \mathrm{mL}^{\mathrm{a}}$ & \\
\hline & C. glabrata & $200 \mathrm{mg} / \mathrm{mL}^{\mathrm{a}}$ & \\
\hline & C. krusei & $200 \mathrm{mg} / \mathrm{mL}^{\mathrm{a}}$ & \\
\hline Quercus infectoria & C. albicans & $200 \mathrm{mg} / \mathrm{mL}^{\mathrm{a}}$ & Qader et al. (2013) \\
\hline & C. dublicans & $200 \mathrm{mg} / \mathrm{mL}^{\mathrm{a}}$ & \\
\hline & C. glabrata & $200 \mathrm{mg} / \mathrm{mL}^{\mathrm{a}}$ & \\
\hline & C. krusei & $200 \mathrm{mg} / \mathrm{mL}^{\mathrm{a}}$ & \\
\hline & C. tropicalis & $200 \mathrm{mg} / \mathrm{mL}^{\mathrm{a}}$ & \\
\hline Rhus angustifolia & C. albicans & $200 \mathrm{mg} / \mathrm{mL}^{\mathrm{a}}$ & Qader et al. (2013) \\
\hline & C. dublicans & $200 \mathrm{mg} / \mathrm{mL}^{\mathrm{a}}$ & \\
\hline & C. glabrata & $200 \mathrm{mg} / \mathrm{mL}^{\mathrm{a}}$ & \\
\hline & C. krusei & $200 \mathrm{mg} / \mathrm{mL}^{\mathrm{a}}$ & \\
\hline & C. tropicalis & $200 \mathrm{mg} / \mathrm{mL}^{\mathrm{a}}$ & \\
\hline Ribes nigrum & C. glabrata & $3.85 \mathrm{mg} / \mathrm{mL}$ & Krisch et al. (2008) \\
\hline & C. inconspicua & $2.82 \mathrm{mg} / \mathrm{mL}$ & \\
\hline & C. lipolytica & $5.15 \mathrm{mg} / \mathrm{mL}$ & \\
\hline & C. norvegica & $3.62 \mathrm{mg} / \mathrm{mL}$ & \\
\hline & C. parapsilosis & $2.76 \mathrm{mg} / \mathrm{mL}$ & \\
\hline & C. tropicalis & $3.75 \mathrm{mg} / \mathrm{mL}$ & \\
\hline & C. zeylanoides & $1.83 \mathrm{mg} / \mathrm{mL}$ & \\
\hline Rosmarinus officinalis & C. albicans & $300 \mathrm{mg} / \mathrm{mL}^{\mathrm{a}}$ & Abdul et al. (2012) \\
\hline Salvia officinalis & C. albicans & $15 \mathrm{mg} / \mathrm{mL}$ & Jasim and Al-khaliq (2011) \\
\hline & C. parapsilosis & $2.5 \mathrm{mg} / \mathrm{mL}$ & Martins et al. (2015) \\
\hline & C. tropicalis & $1.25 \mathrm{mg} / \mathrm{mL}$ & (Martins et al., 2015) \\
\hline Sapindus saponaria & C. parapsilosis & $0.16 \mathrm{mg} / \mathrm{mL}$ & Tsuzuki et al. (2007) \\
\hline Scutellaria baicalensis & C. albicans & $2.5 \mathrm{mg} / \mathrm{mL}$ & Wong and Tsang (2009) \\
\hline & & $5 \mathrm{mg} / \mathrm{mL}$ & \\
\hline Solanum nigrum & C. albicans & $0.2 \mathrm{mg} / \mathrm{mL}$ & Shamim et al. (2004) \\
\hline & C. grabrata & $1 \mathrm{mg} / \mathrm{mL}$ & \\
\hline & C. tropicalis & $0.2 \mathrm{mg} / \mathrm{mL}$ & \\
\hline
\end{tabular}


Table 2 (Continued)

\begin{tabular}{|c|c|c|c|}
\hline Plant source & Candida species & MIC & References \\
\hline \multirow[t]{5}{*}{ Solidago gigantean } & C. albicans & $0.8 \mathrm{mg} / \mathrm{mL}$ & \multirow[t]{5}{*}{ Webster et al. (2008) } \\
\hline & C. glabrata & $0.1 \mathrm{mg} / \mathrm{mL}$ & \\
\hline & C. krusei & $0.8 \mathrm{mg} / \mathrm{mL}$ & \\
\hline & C. lusitaniae & $1.6 \mathrm{mg} / \mathrm{mL}$ & \\
\hline & C. tropicalis & $12.5 \mathrm{mg} / \mathrm{mL}$ & \\
\hline Sonneratia alba & C. albicans & $0.25 \mathrm{mg} / \mathrm{mL}$ & Kaewpiboon et al. (2012) \\
\hline Sonneratia caseolaris & C. albicans & $0.125 \mathrm{mg} / \mathrm{mL}$ & Kaewpiboon et al. (2012) \\
\hline \multirow[t]{4}{*}{ Syzygium aromaticum } & C. albicans & $0.782 \mathrm{mg} / \mathrm{mL}$ & \multirow[t]{4}{*}{ (Kim and Lee (2012) } \\
\hline & C. glabrata & $0.025 \mathrm{mg} / \mathrm{mL}$ & \\
\hline & C. krusei & $0.098 \mathrm{mg} / \mathrm{mL}$ & \\
\hline & C. tropicalis & $0.003125 \mathrm{mg} / \mathrm{mL}$ & \\
\hline \multirow[t]{4}{*}{ Thymus kotschyana } & C. albicanus & $200 \mathrm{mg} / \mathrm{mL}^{\mathrm{a}}$ & \multirow[t]{4}{*}{ Qader et al. (2013) } \\
\hline & C. glabrata & $200 \mathrm{mg} / \mathrm{mL}^{\mathrm{a}}$ & \\
\hline & C. krusei & $200 \mathrm{mg} / \mathrm{mL}^{\mathrm{a}}$ & \\
\hline & C. tropicalis & $200 \mathrm{mg} / \mathrm{mL}^{\mathrm{a}}$ & \\
\hline Thymus vulgaris & C. albicans & $300 \mathrm{mg} / \mathrm{mL}^{\mathrm{a}}$ & Abdul et al. (2012) \\
\hline \multirow[t]{5}{*}{ Zingiber officinalis } & C. albicanus & $200 \mathrm{mg} / \mathrm{mL}^{\mathrm{a}}$ & \multirow[t]{5}{*}{ Qader et al. (2013) } \\
\hline & C. dublicans & $200 \mathrm{mg} / \mathrm{mL}^{\mathrm{a}}$ & \\
\hline & C. glabrata & $200 \mathrm{mg} / \mathrm{mL}^{\mathrm{a}}$ & \\
\hline & C. krusei & $200 \mathrm{mg} / \mathrm{mL}^{\mathrm{a}}$ & \\
\hline & C. tropicalis & $200 \mathrm{mg} / \mathrm{mL}^{\mathrm{a}}$ & \\
\hline
\end{tabular}

MIC - minimum inhibitory concentration.

a Concentration used to evaluate antifungal activity by disc diffusion.

extracts (Table 7). For example, hydroalcoholic extracts of Cassia fistula $(\mathrm{MIC}=0.025 \mathrm{mg} / \mathrm{mL}$ ), Emblica officinalis $(\mathrm{MIC}=0.1024 \mathrm{mg} / \mathrm{mL}$ ), Glycyrrhiza glabra (MIC $=0.512 \mathrm{mg} / \mathrm{mL}$ ) and Punica granatum $(\mathrm{MIC}=0.0039 \mathrm{mg} / \mathrm{mL}$ ) presented a higher antifungal effect, against C. albicans, than the extracts of the same plant species prepared with a single solvent. The only exceptions were the aqueous extracts of Sapindus saponaria (MIC $=0.16 \mathrm{mg} / \mathrm{mL}$ ), against $C$. parapsilosis, and Syzygium aromaticum (MIC $=0.003125 \mathrm{mg} / \mathrm{mL}$ ), against $C$. tropicalis, that gave a higher effect than its hydroalcoholic extracts, and the methanolic extract of Salvia officinalis ( $\mathrm{MIC}=0.16 \mathrm{mg} / \mathrm{mL}$ ) that evidenced a higher antifungal potential, against $C$. tropicalis, than the hydroalcoholic $(\mathrm{MIC}=1.25 \mathrm{mg} / \mathrm{mL}$ ) and aqueous ( $\mathrm{MIC}=1.25 \mathrm{mg} / \mathrm{mL}$ ) extracts. Notwithstanding, the number of studies that report antifungal properties of hydroalcoholic extracts is lower than the studies using methanol, ethanol or water.

Comparing the antifungal potential of the extracts prepared with a unique solvent, the methanolic extracts (Table 5) showed the lowest MICs for the majority of the Candida species, followed by aqueous (Table 2) and ethanolic (Table 3) extracts. In particular, Adiantum capillus veneris, Artemisia herba-alba, Capparis spinosa, Eucalyptus sideroxylon, E. torquata, Glycyrrhiza glabra, Laurus nobilis, Pimpinella anisum and Salvia officinalis methanolic extracts showed higher antifungal potential than their aqueous and ethanolic extracts. On the other hand, Acorus calamus, Funtumia elastica, Hyssopus officinalis subsp. pilifer, Murraya koenigii, Ribes nigrum and Zingiber officinalis aqueous extracts evidenced most pronounced antifungal effects than their ethanolic and methanolic extracts. Allium sativum, Aloe barbadensis, Aloe excelsa, Cinnamomum spp. and Solanum nigrum aqueous and ethanolic extracts evidenced similar potential against Candida species. Lastly, Camellia sinensis, Cassia alata, Mallotus oppositifolius, Morinda morindoides, Origanum vulgare, Plantago media, Punica granatum, Quercus infectoria, Rhus angustifolia, Sclerocarya birrea and Thymus kotschyana ethanolic extracts evidenced higher antifungal potential than aqueous and methanolic extracts.

In specific plant species, the tendency described was modified. For example, the methanol-dichloromethane extract of Andrographis paniculata showed a higher antifungal potential than their ethanolic and methanolic extracts; ethanol, acetone, diethyl ether and ethyl acetate extracts of Origanum vulgare, as well as aqueous and ethyl acetate extracts of Hyssopus officinalis subsp.
Pilifer and aqueous and hydroalcoholic extracts of Salvia officinalis showed similar antifungal effects. Furthermore, Crossandra infundibuliformis ethyl acetate and methanol extracts showed similar effects, whereas Gonzalagunia rosea and Montanoa methanolic extracts showed higher antifungal potential than the corresponding methanol:dichloromethane extracts; the acetone extract of Paullinia cupana seems to be more effective than the ethanolic extract against $C$. albicans, but due to the differences in the units used to express the results, it is difficult to compare both values.

Analysing the antifungal activity against specific Candida species, hydroalcoholic extracts showed the most pronounced properties against $C$. albicans, followed by methanolic and methanol-dichloromethane extracts. Punica granatum hydroalcoholic (ethanol:water, 50:50 v/v) extract revealed the highest antifungal activity ( $\mathrm{MIC}=0.0039 \mathrm{mg} / \mathrm{mL}$ ). In general, methanolic extracts followed by aqueous and hydroalcoholic extracts, showed a higher antifungal potential against $C$. glabrata than the ethanolic and methanol-dichloromethane extracts. Dorstenia turbinata $(0.039 \mathrm{mg} / \mathrm{mL})$ and Thymbra spicata $(0.01 \mathrm{mg} / \mathrm{mL})$ methanolic extracts evidenced the most pronounced effects. For C. guilliermondii, the hydroalcoholic extract of Syzygium aromaticum (MICs $=0.05 \mathrm{mg} / \mathrm{mL}$ ) was the most effective preparations, but Punica granatum ethanolic extract $(\mathrm{MIC}=0.125 \mathrm{mg} / \mathrm{mL}$ ) also evidenced a lower MIC value. For C. krusei, the methanolic extracts were the most efficient plant extract preparations, followed by ethanolic and aqueous extracts. Thymbra spicata ( $\mathrm{MIC}=0.01 \mathrm{mg} / \mathrm{mL}$ ) and Laurus nobilis ( $\mathrm{MIC}=0.07 \mathrm{mg} / \mathrm{mL}$ ) comprises the methanolic extracts that evidenced highest antifungal activity against $C$. krusei. Concerning $C$. lusitaniae, aqueous extracts presented the highest antifungal potential, followed by ethanolic, methanol-dichloromethane and ethyl acetate extracts. Epilobium augustifolium aqueous extract (MIC $=0.05 \mathrm{mg} / \mathrm{mL}$ ) revealed to be the best against $C$. lusitaniae. On the other hand, to the $C$. parapsilosis, the hydroalcoholic extracts were the most effective preparations, followed by methanolic, aqueous and ethanolic extracts. Punica granatum hydroalcoholic extract $(\mathrm{MIC}=0.0039 \mathrm{mg} / \mathrm{mL}$ ) presented the most pronounced antifungal potential against $C$. parapsilosis. Lastly, aqueous extracts were the most efficient plant extract preparations against $C$. tropicalis, followed by methanolic, methanol-dichloromethane and hydroalcoholic extracts. Syzygium aromaticum $(\mathrm{MIC}=0.003125 \mathrm{mg} / \mathrm{mL}$ ) aqueous extract revealing the highest potential. 
Table 3

Activity of ethanolic extracts against Candida species.

\begin{tabular}{|c|c|c|c|}
\hline Plant source & Candida species & MIC & References \\
\hline Achillea biebersteinii & C. albicans & $100 \mathrm{mg} / \mathrm{mL}$ & Hassawi and Kharma (2006) \\
\hline Achillea fragantissima & C. albicans & $150 \mathrm{mg} / \mathrm{mL}$ & Hassawi and Kharma (2006) \\
\hline Acorus calamus & C. albicans & $28.80 \mathrm{mg} / \mathrm{mL}$ & Thirach et al. (2003) \\
\hline Adiantum capillus veneris & C. albicans & $1 \mathrm{mg} / \mathrm{mL}^{\mathrm{a}}$ & Ishaq et al. (2014) \\
\hline Allium cepa & C. albicans & $10 \mathrm{mg} / \mathrm{mL}^{\mathrm{a}}$ & Doddanna et al. (2013) \\
\hline Allium sativum & C. albicans & $1 \mathrm{mg} / \mathrm{mL}^{\mathrm{a}}$ & Joe et al. (2009) \\
\hline \multirow[t]{3}{*}{ Allium sativum } & C. albicans & $0.2 \mathrm{mg} / \mathrm{mL}$ & Shamim et al. (2004) \\
\hline & C. grabrata & $0.2 \mathrm{mg} / \mathrm{mL}$ & \\
\hline & C. tropicalis & $0.2 \mathrm{mg} / \mathrm{mL}$ & \\
\hline \multirow[t]{3}{*}{ Aloe barbadensis } & C. albicans & $1 \mathrm{mg} / \mathrm{mL}$ & Shamim et al. (2004) \\
\hline & C. grabrata & $1 \mathrm{mg} / \mathrm{mL}$ & \\
\hline & C. tropicalis & $0.2 \mathrm{mg} / \mathrm{mL}$ & \\
\hline \multirow[t]{2}{*}{ Aloe excelsa } & C. albicans & $1 \mathrm{mg} / \mathrm{mL}$ & Coopoosamy and \\
\hline & C. tropicalis & $0.2 \mathrm{mg} / \mathrm{mL}$ & Magwa (2007) \\
\hline Aloe vera & C. albicans & $10 \mathrm{mg} / \mathrm{mL}^{\mathrm{a}}$ & Doddanna et al. (2013) \\
\hline Andrographis paniculata & C. albicans & Viability: $103.85 \%$ & Wong and Tsang (2009) \\
\hline Anthemis pseudocotula & C. albicans & $150 \mathrm{mg} / \mathrm{mL}$ & Hassawi and Kharma (2006) \\
\hline Artemisia herba-alba & C. albicans & $100 \mathrm{mg} / \mathrm{mL}$ & Hassawi and Kharma (2006) \\
\hline Ballota undulate & C. albicans & $25 \mathrm{mg} / \mathrm{mL}$ & Hashem (2011) \\
\hline Camellia sinensis & C. albicans & $10 \mathrm{mg} / \mathrm{mL}^{\mathrm{a}}$ & Doddanna et al. (2013) \\
\hline Capparis spinosa & C. albicans & $100 \mathrm{mg} / \mathrm{mL}^{\mathrm{a}}$ & Mandeel and Taha (2005) \\
\hline Capsicum annum & C. albicans & $17.5 \mathrm{mg} / \mathrm{mL}$ & Ertürk (2006) \\
\hline Cassia alata & C. albicans & $5.60 \mathrm{mg}$ & Timothy et al. (2012) \\
\hline \multirow[t]{6}{*}{ Cassia siamea } & C. albicans & $100 \mathrm{mg} / \mathrm{mL}$ & Prabhakar et al. (2008) \\
\hline & C. glabrata & $100 \mathrm{mg} / \mathrm{mL}$ & \\
\hline & C. guilliermondii & $100 \mathrm{mg} / \mathrm{mL}$ & \\
\hline & C. krusei & $100 \mathrm{mg} / \mathrm{mL}$ & \\
\hline & C. parapsilosis & $100 \mathrm{mg} / \mathrm{mL}$ & \\
\hline & C. tropicalis & $100 \mathrm{mg} / \mathrm{mL}$ & \\
\hline \multirow[t]{6}{*}{ Caulerpa scalpelliformis } & C. albicans & $100 \mathrm{mg} / \mathrm{mL}$ & Prabhakar et al. (2008) \\
\hline & C. glabrata & $100 \mathrm{mg} / \mathrm{mL}$ & \\
\hline & C. guilliermondii & $100 \mathrm{mg} / \mathrm{mL}$ & \\
\hline & C. krusei & $100 \mathrm{mg} / \mathrm{mL}$ & \\
\hline & C. parapsilosis & $100 \mathrm{mg} / \mathrm{mL}$ & \\
\hline & C. tropicalis & $100 \mathrm{mg} / \mathrm{mL}$ & \\
\hline \multirow[t]{5}{*}{ Cinnamomum sp. } & C. albicanus & $200 \mathrm{mg} / \mathrm{mL}^{\mathrm{a}}$ & Qader et al. (2013) \\
\hline & C. dublicans & $200 \mathrm{mg} / \mathrm{mL}^{\mathrm{a}}$ & \\
\hline & C. glabrata & $200 \mathrm{mg} / \mathrm{mL}^{\mathrm{a}}$ & \\
\hline & C. krusei & $200 \mathrm{mg} / \mathrm{mL}^{\mathrm{a}}$ & \\
\hline & C. tropicalis & $200 \mathrm{mg} / \mathrm{mL}^{\mathrm{a}}$ & \\
\hline Cleome amplyocarpa & C. albicans & $75 \mathrm{mg} / \mathrm{mL}$ & Hashem (2011) \\
\hline Colutea arborescens & C. albicans & $12.5 \mathrm{mg} / \mathrm{mL}$ & Ertürk (2006) \\
\hline Convolvulus arvensis & C. albicans & $100 \mathrm{mg} / \mathrm{mL}^{\mathrm{a}}$ & Mandeel and Taha (2005) \\
\hline Convolvulus arvensis & C. albicans & $150 \mathrm{mg} / \mathrm{mL}$ & Hassawi and Kharma (2006) \\
\hline Coscinium fenestratum & C. albicans & $0.5 \mathrm{mg} / \mathrm{mL}$ & Kaewpiboon et al. (2012) \\
\hline Crataegus oxyacantha & C. albicans & $10 \mathrm{mg} / \mathrm{mL}^{\mathrm{a}}$ & Kostić et al. (2012) \\
\hline Cressa cretica & C. albicans & $100 \mathrm{mg} / \mathrm{mL}^{\mathrm{a}}$ & Mandeel and Taha (2005) \\
\hline Cuminum cyminum & C. albicans & $15 \mathrm{mg} / \mathrm{mL}$ & Ertürk (2006) \\
\hline \multirow{2}{*}{ Cynara scolymus } & C. albicans & $12.5 \mathrm{mg} / \mathrm{mL}$ & Zhu et al. (2005) \\
\hline & C. lusitaniae & $12.5 \mathrm{mg} / \mathrm{mL}$ & \\
\hline Dianthus coryophyllum & C. albicans & $20 \mathrm{mg} / \mathrm{mL}$ & Ertürk (2006) \\
\hline Emex spinosa & C. albicans & $100 \mathrm{mg} / \mathrm{mL}^{\mathrm{a}}$ & Mandeel and Taha (2005) \\
\hline Erica arborea & C. albicans & $2.5 \mathrm{mg} / \mathrm{mL}$ & Ertürk (2006) \\
\hline Eugenia caryophyllus & C. albicans & $17.41 \mathrm{mg} / \mathrm{mL}$ & Thirach et al. (2003) \\
\hline Eugenia uniflora & C. krusei & $0.25 \mathrm{mg} / \mathrm{mL}$ & Correa-Royero et al. (2010) \\
\hline Fagonia indica & C. albicans & $100 \mathrm{mg} / \mathrm{mL}^{\mathrm{a}}$ & Mandeel and Taha (2005) \\
\hline Fraxinus rhynchophylla & C. albicans & Viability: $77.56 \%$ & Wong and Tsang (2009) \\
\hline Funtumia elastica & C. albicans & $100 \mathrm{mg} / \mathrm{mL}^{\mathrm{a}}$ & Adekunle and Ikumapayi (2006) \\
\hline \multirow[t]{4}{*}{ Glycyrrhiza glabra } & C. albicans & $1 \mathrm{mg} / \mathrm{mL}$ & Fatima et al. (2009) \\
\hline & C. krusei & $2 \mathrm{mg} / \mathrm{mL}$ & \\
\hline & C. nerformans & $0.5 \mathrm{mg} / \mathrm{mL}$ & \\
\hline & C. pseudotropicalis & $1 \mathrm{mg} / \mathrm{mL}$ & \\
\hline Heliotropium curassavicum & C. albicans & $100 \mathrm{mg} / \mathrm{mL}^{\mathrm{a}}$ & Mandeel and Taha (2005) \\
\hline Illicium verum & C. albicans & $16 \mathrm{mg} / \mathrm{mL}$ & Yazdani et al. (2009) \\
\hline Isatis indigotica & C. albicans & Viability: $94.05 \%$ & Wong and Tsang (2009) \\
\hline Juniperus oxycedrus & C. albicans & $5 \mathrm{mg} / \mathrm{mL}$ & Ertürk (2006) \\
\hline Lantana fucata & C. krusei & $0.5 \mathrm{mg} / \mathrm{mL}$ & Correa-Royero et al. (2010) \\
\hline Laurus nobilis & C. albicans & $5 \mathrm{mg} / \mathrm{mL}$ & Ertürk (2006) \\
\hline Mahonia aquifolium & C. tropicalis & $1000 \mu \mathrm{g} / \mathrm{mL}$ & Volleková et al. (2003) \\
\hline Mallotus oppositifolius & C. albicans & $100 \mathrm{mg} / \mathrm{mL}^{\mathrm{a}}$ & Adekunle and Ikumapayi (2006) \\
\hline Melissa officinalis & C. albicans & $25 \mathrm{mg} / \mathrm{mL}$ & Ertürk (2006) \\
\hline Mentha piperita & C. albicans & $5 \mathrm{mg} / \mathrm{mL}$ & Ertürk (2006) \\
\hline Mentha piperita & C. albicans & $10 \mathrm{mg} / \mathrm{mL}^{\mathrm{a}}$ & Doddanna et al. (2013) \\
\hline Morinda morindoides & C. albicans & $31.25 \mathrm{mg} / \mathrm{mL}$ & Touré et al. (2011) \\
\hline Morinda royoc & C. krusei & $0.25 \mathrm{mg} / \mathrm{mL}$ & Correa-Royero et al. (2010) \\
\hline
\end{tabular}


Table 3 (Continued)

\begin{tabular}{|c|c|c|c|}
\hline Plant source & Candida species & MIC & References \\
\hline Murraya koenigii & C. albicans & $10 \mathrm{mg} / \mathrm{mL}^{\mathrm{a}}$ & Doddanna et al. (2013) \\
\hline Myrcia cucullata & C. krusei & $0.03125 \mathrm{mg} / \mathrm{mL}$ & Correa-Royero et al. (2010) \\
\hline \multirow[t]{6}{*}{ Odina wodier } & C. albicans & $500 \mathrm{mg} / \mathrm{mL}$ & Prabhakar et al. (2008) \\
\hline & C. glabrata & $500 \mathrm{mg} / \mathrm{mL}$ & \\
\hline & C. guilliermondii & $500 \mathrm{mg} / \mathrm{mL}$ & \\
\hline & C. krusei & $500 \mathrm{mg} / \mathrm{mL}$ & \\
\hline & C. parapsilosis & $500 \mathrm{mg} / \mathrm{mL}$ & \\
\hline & C. tropicalis & $500 \mathrm{mg} / \mathrm{mL}$ & \\
\hline Origanum vulgare & C. albicans & $5 \mathrm{mg} / \mathrm{mL}$ & Ličina et al. (2013) \\
\hline Paullinia cupana & C. albicans & $0.5 \mathrm{mg} / \mathrm{mL}$ & Basile et al. (2013) \\
\hline \multirow[t]{5}{*}{ Pimpinella anisum } & C. albicans & $19 \%, v / v$ & Kosalec et al. (2005) \\
\hline & C. krusei & $20 \%, v / v$ & \\
\hline & C. parapsilosis & $18 \%, v / v$ & \\
\hline & C. pseudotropicalis & $17 \%, v / v$ & \\
\hline & C. tropicalis & $18 \%, v / v$ & \\
\hline \multirow[t]{2}{*}{ Piper nigrum } & C. albicans & $12.5 \mathrm{mg} / \mathrm{mL}$ & Ertürk (2006) \\
\hline & & $1.5 \mathrm{mg} / \mathrm{mL}^{\mathrm{a}}$ & Joe et al. (2009) \\
\hline \multirow[t]{3}{*}{ Piper regnellii } & C. albicans & $0.125 \mathrm{mg} / \mathrm{mL}$ & Pessini et al. (2005) \\
\hline & C. krusei & $0.5 \mathrm{mg} / \mathrm{mL}$ & \\
\hline & C. parapsilosis & $0.5 \mathrm{mg} / \mathrm{mL}$ & \\
\hline Plantago lanceolata (France) & C. albicans & $200 \mathrm{mg} / \mathrm{mL}$ & Hassawi and Kharma (2006) \\
\hline \multirow[t]{5}{*}{ Plantago media } & C. albicanus & $200 \mathrm{mg} / \mathrm{mL}^{\mathrm{a}}$ & Qader et al. (2013) \\
\hline & C. dublicans & $200 \mathrm{mg} / \mathrm{mL}^{\mathrm{a}}$ & \\
\hline & C. glabrata & $200 \mathrm{mg} / \mathrm{mL}^{\mathrm{a}}$ & \\
\hline & C. krusei & $200 \mathrm{mg} / \mathrm{mL}^{\mathrm{a}}$ & \\
\hline & C. tropicalis & $200 \mathrm{mg} / \mathrm{mL}^{\mathrm{a}}$ & \\
\hline Pluchea ovalis & C. albicans & $100 \mathrm{mg} / \mathrm{mL}^{\mathrm{a}}$ & Mandeel and Taha (2005) \\
\hline \multirow[t]{10}{*}{ Punica granatum } & C. albicans & $0.25 \mathrm{mg} / \mathrm{mL}$ & Anibal et al. (2013) \\
\hline & C. dubliniensis & $0.25 \mathrm{mg} / \mathrm{mL}$ & \\
\hline & C. glabrata & $0.25 \mathrm{mg} / \mathrm{mL}$ & \\
\hline & C. guilliermondii & $0.125 \mathrm{mg} / \mathrm{mL}$ & \\
\hline & C. krusei & $0.125 \mathrm{mg} / \mathrm{mL}$ & \\
\hline & C. lusitaniae & $0.125 \mathrm{mg} / \mathrm{mL}$ & \\
\hline & C. parapsilosis & $0.125 \mathrm{mg} / \mathrm{mL}$ & \\
\hline & C. rugosa & $0.125 \mathrm{mg} / \mathrm{mL}$ & \\
\hline & C. tropicalis & $0.25 \mathrm{mg} / \mathrm{mL}$ & \\
\hline & C. utilis & $0.25 \mathrm{mg} / \mathrm{mL}$ & \\
\hline \multirow{5}{*}{ Punica granatum } & C. albicanus & $200 \mathrm{mg} / \mathrm{mL}^{\mathrm{a}}$ & Qader et al. (2013) \\
\hline & C. dublicans & $200 \mathrm{mg} / \mathrm{mL}^{\mathrm{a}}$ & \\
\hline & C. glabrata & $200 \mathrm{mg} / \mathrm{mL}^{\mathrm{a}}$ & \\
\hline & C. krusei & $200 \mathrm{mg} / \mathrm{mL}^{\mathrm{a}}$ & \\
\hline & C. tropicalis & $200 \mathrm{mg} / \mathrm{mL}^{\mathrm{a}}$ & \\
\hline \multirow[t]{5}{*}{ Quercus infectoria } & C. albicanus & $200 \mathrm{mg} / \mathrm{mL}^{\mathrm{a}}$ & Qader et al. (2013) \\
\hline & C. dublicans & $200 \mathrm{mg} / \mathrm{mL}^{\mathrm{a}}$ & \\
\hline & C. glabrata & $200 \mathrm{mg} / \mathrm{mL}^{\mathrm{a}}$ & \\
\hline & C. krusei & $200 \mathrm{mg} / \mathrm{mL}^{\mathrm{a}}$ & \\
\hline & C. tropicalis & $200 \mathrm{mg} / \mathrm{mL}^{\mathrm{a}}$ & \\
\hline \multirow{5}{*}{ Rhus angustifolia } & C. albicanus & $200 \mathrm{mg} / \mathrm{mL}^{\mathrm{a}}$ & Qader et al. (2013) \\
\hline & C. dublicans & $200 \mathrm{mg} / \mathrm{mL}^{\mathrm{a}}$ & \\
\hline & C. glabrata & $200 \mathrm{mg} / \mathrm{mL}^{\mathrm{a}}$ & \\
\hline & C. krusei & $200 \mathrm{mg} / \mathrm{mL}^{\mathrm{a}}$ & \\
\hline & C. tropicalis & $200 \mathrm{mg} / \mathrm{mL}^{\mathrm{a}}$ & \\
\hline Rhus coriaria & C. albicans & $15 \mathrm{mg} / \mathrm{mL}$ & Ertürk (2006) \\
\hline Sargassum wightii & C. albicans & $100 \mathrm{mg} / \mathrm{mL}$ & Prabhakar et al. (2008) \\
\hline & C. glabrata & $100 \mathrm{mg} / \mathrm{mL}$ & \\
\hline & C. guilliermondii & $100 \mathrm{mg} / \mathrm{mL}$ & \\
\hline & C. krusei & $100 \mathrm{mg} / \mathrm{mL}$ & \\
\hline & C. parapsilosis & $100 \mathrm{mg} / \mathrm{mL}$ & \\
\hline & C. tropicalis & $100 \mathrm{mg} / \mathrm{mL}$ & \\
\hline Sclerocarya birrea & C. parapsilosis & $0.21 \mathrm{mg} / \mathrm{mL}$ & Masoko et al. (2008) \\
\hline Solanum nigrum & C. albicans & $0.2 \mathrm{mg} / \mathrm{mL}$ & Shamim et al. (2004) \\
\hline & C. grabrata & $1 \mathrm{mg} / \mathrm{mL}$ & \\
\hline & C. tropicalis & $0.2 \mathrm{mg} / \mathrm{mL}$ & \\
\hline Syzygium jambolanum & C. albicans & $100 \mathrm{mg} / \mathrm{mL}$ & Prabhakar et al. (2008) \\
\hline & C. glabrata & $100 \mathrm{mg} / \mathrm{mL}$ & \\
\hline & C. guilliermondii & $100 \mathrm{mg} / \mathrm{mL}$ & \\
\hline & C. krusei & $100 \mathrm{mg} / \mathrm{mL}$ & \\
\hline & C. parapsilosis & $100 \mathrm{mg} / \mathrm{mL}$ & \\
\hline & C. tropicalis & $100 \mathrm{mg} / \mathrm{mL}$ & \\
\hline Tamarix arabica & C. albicans & $100 \mathrm{mg} / \mathrm{mL}^{\mathrm{a}}$ & (Mandeel and Taha, 2005) \\
\hline Thymus kotschyana & C. albicanus & $200 \mathrm{mg} / \mathrm{mL}^{\mathrm{a}}$ & (Qader et al., 2013) \\
\hline & C. dublicans & $200 \mathrm{mg} / \mathrm{mL}^{\mathrm{a}}$ & \\
\hline & C. glabrata & $200 \mathrm{mg} / \mathrm{mL}^{\mathrm{a}}$ & \\
\hline & C. krusei & $200 \mathrm{mg} / \mathrm{mL}^{\mathrm{a}}$ & \\
\hline & C. tropicalis & $200 \mathrm{mg} / \mathrm{mL}^{\mathrm{a}}$ & \\
\hline Zingiber officinale & C. albicans & $1 \mathrm{mg} / \mathrm{mL}^{\mathrm{a}}$ & (Joe et al., 2009) \\
\hline
\end{tabular}


Table 3 (Continued)

\begin{tabular}{|c|c|c|c|}
\hline Plant source & Candida species & MIC & References \\
\hline \multirow[t]{5}{*}{ Zingiber officinalis } & C. albicanus & $200 \mathrm{mg} / \mathrm{mL}^{\mathrm{a}}$ & (Qader et al., 2013) \\
\hline & C. dublicans & $200 \mathrm{mg} / \mathrm{mL}^{\mathrm{a}}$ & \\
\hline & C. glabrata & $200 \mathrm{mg} / \mathrm{mL}^{\mathrm{a}}$ & \\
\hline & C. krusei & $200 \mathrm{mg} / \mathrm{mL}^{\mathrm{a}}$ & \\
\hline & C. tropicalis & $200 \mathrm{mg} / \mathrm{mL}^{\mathrm{a}}$ & \\
\hline
\end{tabular}

MIC - minimum inhibitory concentration.

a Concentration used to evaluate antifungal activity by disc diffusion.

Table 4

Activity of methanol-dichloromethane extracts against Candida species.

\begin{tabular}{|c|c|c|c|}
\hline Plant source & Species & MIC & References \\
\hline \multirow[t]{7}{*}{ Aframomum citratum } & C. albicans & $3.12 \mathrm{mg} / \mathrm{mL}$ & Dzoyem et al. (2014) \\
\hline & C. glabrata & $4.68 \mathrm{mg} / \mathrm{mL}$ & \\
\hline & C. guilliermondii & $3.12 \mathrm{mg} / \mathrm{mL}$ & \\
\hline & C. krusei & $0.39 \mathrm{mg} / \mathrm{mL}$ & \\
\hline & C. lusitaniae & $0.78 \mathrm{mg} / \mathrm{mL}$ & \\
\hline & C. parapsilosis & $6.25 \mathrm{mg} / \mathrm{mL}$ & \\
\hline & C. tropicalis & $6.25 \mathrm{mg} / \mathrm{mL}$ & \\
\hline \multirow[t]{6}{*}{ Aframomum melegueta } & C. albicans & $6.25 \mathrm{mg} / \mathrm{mL}$ & Dzoyem et al. (2014) \\
\hline & C. guilliermondii & $1.56 \mathrm{mg} / \mathrm{mL}$ & \\
\hline & C. krusei & $6.25 \mathrm{mg} / \mathrm{mL}$ & \\
\hline & C. lusitaniae & $3.12 \mathrm{mg} / \mathrm{mL}$ & \\
\hline & C. parapsilosis & $6.25 \mathrm{mg} / \mathrm{mL}$ & \\
\hline & C. tropicalis & $1.56 \mathrm{mg} / \mathrm{mL}$ & \\
\hline Andrographis & C. albicans & $0.1 \mathrm{mg} / \mathrm{mL}$ & Sule et al. (2012) \\
\hline \multirow[t]{2}{*}{ paniculata $^{\mathrm{a}}$} & C. krusei & $0.25 \mathrm{mg} / \mathrm{mL}$ & \\
\hline & C. tropicalis & $0.1 \mathrm{mg} / \mathrm{mL}$ & \\
\hline Bielschmiedia & C. albicans & $3.12 \mathrm{mg} / \mathrm{mL}$ & Dzoyem et al. (2014) \\
\hline \multirow{4}{*}{ cinnamomea } & C. glabrata & $6.25 \mathrm{mg} / \mathrm{mL}$ & \\
\hline & C. guilliermondii & $6.25 \mathrm{mg} / \mathrm{mL}$ & \\
\hline & C. lusitaniae & $3.12 \mathrm{mg} / \mathrm{mL}$ & \\
\hline & C. tropicalis & $3.12 \mathrm{mg} / \mathrm{mL}$ & \\
\hline Cinnamomum & C. albicans & $3.12 \mathrm{mg} / \mathrm{mL}$ & Dzoyem et al. (2014) \\
\hline \multirow[t]{6}{*}{ zeylanicum } & C. glabrata & $3.12 \mathrm{mg} / \mathrm{mL}$ & \\
\hline & C. guilliermondii & $0.78 \mathrm{mg} / \mathrm{mL}$ & \\
\hline & C. krusei & $0.78 \mathrm{mg} / \mathrm{mL}$ & \\
\hline & C. lusitaniae & $0.78 \mathrm{mg} / \mathrm{mL}$ & \\
\hline & C. parapsilosis & $3.12 \mathrm{mg} / \mathrm{mL}$ & \\
\hline & C. tropicalis & $0.097 \mathrm{mg} / \mathrm{mL}$ & \\
\hline Dichrostachys & C. albicans & $0.39 \mathrm{mg} / \mathrm{mL}$ & Dzoyem et al. (2014) \\
\hline \multirow[t]{6}{*}{ glomerata } & C. glabrata & $3.12 \mathrm{mg} / \mathrm{mL}$ & \\
\hline & C. guilliermondii & $3.12 \mathrm{mg} / \mathrm{mL}$ & \\
\hline & C. krusei & $3.12 \mathrm{mg} / \mathrm{mL}$ & \\
\hline & C. lusitaniae & $0.39 \mathrm{mg} / \mathrm{mL}$ & \\
\hline & C. parapsilosis & $6.25 \mathrm{mg} / \mathrm{mL}$ & \\
\hline & C. tropicalis & $3.12 \mathrm{mg} / \mathrm{mL}$ & \\
\hline \multirow[t]{4}{*}{ Diospyros crassiflora } & C. albicans & $12.5 \mathrm{mg} / \mathrm{mL}$ & Dzoyem et al. (2007) \\
\hline & C. glabrata & $25 \mathrm{mg} / \mathrm{mL}$ & \\
\hline & C. krusei & $12.5 \mathrm{mg} / \mathrm{mL}$ & \\
\hline & C. tropicalis & $12.5 \mathrm{mg} / \mathrm{mL}$ & \\
\hline \multirow[t]{7}{*}{ Dorstenia psilirus } & C. albicans & $0.39 \mathrm{mg} / \mathrm{mL}$ & Dzoyem et al. (2014) \\
\hline & C. glabrata & $3.12 \mathrm{mg} / \mathrm{mL}$ & \\
\hline & C. guilliermondii & $1.56 \mathrm{mg} / \mathrm{mL}$ & \\
\hline & C. krusei & $3.12 \mathrm{mg} / \mathrm{mL}$ & \\
\hline & C. lusitaniae & $3.12 \mathrm{mg} / \mathrm{mL}$ & \\
\hline & C. parapsilosis & $3.12 \mathrm{mg} / \mathrm{mL}$ & \\
\hline & C. tropicalis & $0.39 \mathrm{mg} / \mathrm{mL}$ & \\
\hline \multirow{6}{*}{ Echinops giganteus } & C. albicans & $6.25 \mathrm{mg} / \mathrm{mL}$ & Dzoyem et al. (2014) \\
\hline & C. glabrata & $1.56 \mathrm{mg} / \mathrm{mL}$ & \\
\hline & C. guilliermondii & $3.12 \mathrm{mg} / \mathrm{mL}$ & \\
\hline & C. lusitaniae & $6.25 \mathrm{mg} / \mathrm{mL}$ & \\
\hline & C. parapsilosis & $6.25 \mathrm{mg} / \mathrm{mL}$ & \\
\hline & C. tropicalis & $6.25 \mathrm{mg} / \mathrm{mL}$ & \\
\hline \multirow[t]{5}{*}{ Fagara leprieurii } & C. albicans & $3.12 \mathrm{mg} / \mathrm{mL}$ & Dzoyem et al. (2014) \\
\hline & C. glabrata & $3.12 \mathrm{mg} / \mathrm{mL}$ & \\
\hline & C. krusei & $6.25 \mathrm{mg} / \mathrm{mL}$ & \\
\hline & C. parapsilosis & $6.25 \mathrm{mg} / \mathrm{mL}$ & \\
\hline & C. tropicalis & $6.25 \mathrm{mg} / \mathrm{mL}$ & \\
\hline \multirow[t]{6}{*}{ Fagara macrophylla } & C. albicans & $3.12 \mathrm{mg} / \mathrm{mL}$ & Dzoyem et al. (2014) \\
\hline & C. glabrata & $3.12 \mathrm{mg} / \mathrm{mL}$ & \\
\hline & C. guilliermondii & $1.56 \mathrm{mg} / \mathrm{mL}$ & \\
\hline & C. krusei & $6.25 \mathrm{mg} / \mathrm{mL}$ & \\
\hline & C. lusitaniae & $1.56 \mathrm{mg} / \mathrm{mL}$ & \\
\hline & C. tropicalis & $0.39 \mathrm{mg} / \mathrm{mL}$ & \\
\hline Gonzalagunia rosea ${ }^{\mathrm{a}}$ & C. albicans & $2.50 \mathrm{mg} / \mathrm{mL}$ & Niño et al. (2007) \\
\hline
\end{tabular}


Table 4 (Continued)

\begin{tabular}{|c|c|c|c|}
\hline Plant source & Species & MIC & References \\
\hline \multirow[t]{7}{*}{ Imperata cylindrical } & C. albicans & $6.25 \mathrm{mg} / \mathrm{mL}$ & Dzoyem et al. (2014) \\
\hline & C. glabrata & $3.12 \mathrm{mg} / \mathrm{mL}$ & \\
\hline & C. guilliermondii & $3.12 \mathrm{mg} / \mathrm{mL}$ & \\
\hline & C. krusei & $1.56 \mathrm{mg} / \mathrm{mL}$ & \\
\hline & C. lusitaniae & $3.12 \mathrm{mg} / \mathrm{mL}$ & \\
\hline & C. parapsilosis & $6.25 \mathrm{mg} / \mathrm{mL}$ & \\
\hline & C. tropicalis & $3.12 \mathrm{mg} / \mathrm{mL}$ & \\
\hline Liabum asclepiadeum $^{\mathrm{a}}$ & C. albicans & $0.62 \mathrm{mg} / \mathrm{mL}$ & Niño et al. (2007) \\
\hline \multirow{4}{*}{ Mondia whitei } & C. albicans & $3.12 \mathrm{mg} / \mathrm{mL}$ & Dzoyem et al. (2014) \\
\hline & C. guilliermondii & $6.25 \mathrm{mg} / \mathrm{mL}$ & \\
\hline & C. lusitaniae & $3.12 \mathrm{mg} / \mathrm{mL}$ & \\
\hline & C. tropicalis & $3.12 \mathrm{mg} / \mathrm{mL}$ & \\
\hline \multirow[t]{5}{*}{ Monodora myristica } & C. albicans & $6.25 \mathrm{mg} / \mathrm{mL}$ & Dzoyem et al. (2014) \\
\hline & C. guilliermondii & $3.12 \mathrm{mg} / \mathrm{mL}$ & \\
\hline & C. krusei & $6.25 \mathrm{mg} / \mathrm{mL}$ & \\
\hline & C. lusitaniae & $3.12 \mathrm{mg} / \mathrm{mL}$ & \\
\hline & C. tropicalis & $3.12 \mathrm{mg} / \mathrm{mL}$ & \\
\hline Montanoa $^{\mathrm{a}}$ & C. albicans & $1.25 \mathrm{mg} / \mathrm{mL}$ & Niño et al. (2007) \\
\hline \multirow[t]{7}{*}{ Olax subscorpioidea } & C. albicans & $0.097 \mathrm{mg} / \mathrm{mL}$ & Dzoyem et al. (2014) \\
\hline & C. glabrata & $1.56 \mathrm{mg} / \mathrm{mL}$ & \\
\hline & C. guilliermondii & $0.78 \mathrm{mg} / \mathrm{mL}$ & \\
\hline & C. krusei & $1.56 \mathrm{mg} / \mathrm{mL}$ & \\
\hline & C. lusitaniae & $0.19 \mathrm{mg} / \mathrm{mL}$ & \\
\hline & C. parapsilosis & $0.39 \mathrm{mg} / \mathrm{mL}$ & \\
\hline & C. tropicalis & $0.048 \mathrm{mg} / \mathrm{mL}$ & \\
\hline Pentadiplandra & C. albicans & $3.12 \mathrm{mg} / \mathrm{mL}$ & Dzoyem et al. (2014) \\
\hline \multirow{4}{*}{ brazzeana } & C. glabrata & $6.25 \mathrm{mg} / \mathrm{mL}$ & \\
\hline & C. guilliermondii & $6.25 \mathrm{mg} / \mathrm{mL}$ & \\
\hline & C. lusitaniae & $6.25 \mathrm{mg} / \mathrm{mL}$ & \\
\hline & C. tropicalis & $1.56 \mathrm{mg} / \mathrm{mL}$ & \\
\hline \multirow[t]{6}{*}{ Piper capense } & C. albicans & $3.12 \mathrm{mg} / \mathrm{mL}$ & Dzoyem et al. (2014) \\
\hline & C. guilliermondii & $3.12 \mathrm{mg} / \mathrm{mL}$ & \\
\hline & C. krusei & $3.12 \mathrm{mg} / \mathrm{mL}$ & \\
\hline & C. lusitaniae & $1.56 \mathrm{mg} / \mathrm{mL}$ & \\
\hline & C. parapsilosis & $3.12 \mathrm{mg} / \mathrm{mL}$ & \\
\hline & C. tropicalis & $0.19 \mathrm{mg} / \mathrm{mL}$ & \\
\hline \multirow[t]{5}{*}{ Piper guineense } & C. albicans & $3.12 \mathrm{mg} / \mathrm{mL}$ & Dzoyem et al. (2014) \\
\hline & C. glabrata & $3.12 \mathrm{mg} / \mathrm{mL}$ & \\
\hline & C. lusitaniae & $1.56 \mathrm{mg} / \mathrm{mL}$ & \\
\hline & C. parapsilosis & $6.25 \mathrm{mg} / \mathrm{mL}$ & \\
\hline & C. tropicalis & $3.12 \mathrm{mg} / \mathrm{mL}$ & \\
\hline Schizozygia caffaeoides ${ }^{\mathrm{a}}$ & C. albicans & $2.5 \mathrm{mg} / \mathrm{mL}$ & Kariba et al. (2001) \\
\hline \multirow[t]{7}{*}{ Scorodophloeus zenkeri } & C. albicans & $2.34 \mathrm{mg} / \mathrm{mL}$ & Dzoyem et al. (2014) \\
\hline & C. glabrata & $4.68 \mathrm{mg} / \mathrm{mL}$ & \\
\hline & C. guilliermondii & $6.25 \mathrm{mg} / \mathrm{mL}$ & \\
\hline & C. krusei & $3.90 \mathrm{mg} / \mathrm{mL}$ & \\
\hline & C. lusitaniae & $2.34 \mathrm{mg} / \mathrm{mL}$ & \\
\hline & C. parapsilosis & $6.25 \mathrm{mg} / \mathrm{mL}$ & \\
\hline & C. tropicalis & $4.68 \mathrm{mg} / \mathrm{mL}$ & \\
\hline \multirow[t]{2}{*}{ Solanum melongena } & C. guilliermondii & $6.25 \mathrm{mg} / \mathrm{mL}$ & Dzoyem et al. (2014) \\
\hline & C. tropicalis & $3.12 \mathrm{mg} / \mathrm{mL}$ & \\
\hline \multirow[t]{7}{*}{ Tetrapleura tretraptera } & C. albicans & $3.12 \mathrm{mg} / \mathrm{mL}$ & Dzoyem et al. (2014) \\
\hline & C. glabrata & $6.25 \mathrm{mg} / \mathrm{mL}$ & \\
\hline & C. guilliermondii & $3.12 \mathrm{mg} / \mathrm{mL}$ & \\
\hline & C. krusei & $6.25 \mathrm{mg} / \mathrm{mL}$ & \\
\hline & C. lusitaniae & $3.12 \mathrm{mg} / \mathrm{mL}$ & \\
\hline & C. parapsilosis & $3.12 \mathrm{mg} / \mathrm{mL}$ & \\
\hline & C. tropicalis & $6.25 \mathrm{mg} / \mathrm{mL}$ & \\
\hline \multirow[t]{4}{*}{ Xylopia aethiopica } & C. albicans & $3.12 \mathrm{mg} / \mathrm{mL}$ & Dzoyem et al. (2014) \\
\hline & C. krusei & $3.12 \mathrm{mg} / \mathrm{Ml}$ & \\
\hline & C. parapsilosis & $3.12 \mathrm{mg} / \mathrm{mL}$ & \\
\hline & C. tropicalis & $3.12 \mathrm{mg} / \mathrm{mL}$ & \\
\hline \multirow{7}{*}{ Xylopia parviflora } & C. albicans & $6.25 \mathrm{mg} / \mathrm{mL}$ & Dzoyem et al. (2014) \\
\hline & C. glabrata & $6.25 \mathrm{mg} / \mathrm{mL}$ & \\
\hline & C. guilliermondii & $3.12 \mathrm{mg} / \mathrm{mL}$ & \\
\hline & C. krusei & $3.12 \mathrm{mg} / \mathrm{mL}$ & \\
\hline & C. lusitaniae & $1.56 \mathrm{mg} / \mathrm{mL}$ & \\
\hline & C. parapsilosis & $3.12 \mathrm{mg} / \mathrm{mL}$ & \\
\hline & C. tropicalis & $3.12 \mathrm{mg} / \mathrm{mL}$ & \\
\hline
\end{tabular}

MIC - minimum inhibitory concentration.

a Dichloromethane extracts.

It can be concluded that the type of solvent used in the extraction of the phytochemicals (including phenolic compounds) has influence in the final composition of the extract, and consequently, in the bioactivity. Nevertheless, the Candida species, as also the strains used had also influence in the antifungal results. Several studies have highlighted some aspects involved in the mecha- 
Table 5

Activity of methanolic extracts against Candida species.

\begin{tabular}{|c|c|c|c|}
\hline Plant sources & Candida species & MIC & References \\
\hline Adiantum capillus veneris & C. albicans & $1 \mathrm{mg} / \mathrm{mL}^{\mathrm{a}}$ & Ishaq et al. (2014) \\
\hline \multirow[t]{3}{*}{ Anagyris foetida } & C. albicans & $12.5 \mathrm{mg} / \mathrm{mL}$ & Darwish and Aburjai \\
\hline & C. glabrata & $25 \mathrm{mg} / \mathrm{mL}$ & $(2011)$ \\
\hline & C. krusei & $12.5 \mathrm{mg} / \mathrm{mL}$ & \\
\hline \multirow[t]{3}{*}{ Andrographis paniculata } & C. albicans & $0.2 \mathrm{mg} / \mathrm{mL}$ & Sule et al. (2012) \\
\hline & C. krusei & $0.2 \mathrm{mg} / \mathrm{mL}$ & \\
\hline & C. tropicalis & $0.150 \mathrm{mg} / \mathrm{mL}$ & \\
\hline Arrabidaea chica & C. albicans & $0.5 \mathrm{mg} / \mathrm{mL}$ & Höfling et al. (2011) \\
\hline \multirow[t]{3}{*}{ Artemisia herba-alba } & C. albicans & $6.3 \mathrm{mg} / \mathrm{mL}$ & Darwish and Aburjai \\
\hline & C. glabrata & $25 \mathrm{mg} / \mathrm{mL}$ & $(2011)$ \\
\hline & C. krusei & $12.5 \mathrm{mg} / \mathrm{mL}$ & \\
\hline Caesalpinia pulcherrima & C. albicans & $0.125 \mathrm{mg} / \mathrm{disc}^{\mathrm{a}}$ & Parekh and Chanda (2008) \\
\hline \multirow[t]{3}{*}{ Capparis spinosa } & C. albicans & $12.5 \mathrm{mg} / \mathrm{mL}$ & Darwish and Aburjai \\
\hline & C. glabrata & $12.5 \mathrm{mg} / \mathrm{mL}$ & $(2011)$ \\
\hline & C. krusei & $12.5 \mathrm{mg} / \mathrm{mL}$ & \\
\hline \multirow[t]{3}{*}{ Cassia fistula } & C. albicans & $0.150 \mathrm{mg} / \mathrm{mL}$ & Irshad et al. (2011) \\
\hline & C. glabrata & $0.1 \mathrm{mg} / \mathrm{mL}$ & \\
\hline & C. tropicalis & $0.250 \mathrm{mg} / \mathrm{mL}$ & \\
\hline Centaurium erythraea & C. albicans & $0.1 \mathrm{mg} / \mathrm{mL}$ & Siler et al. (2014) \\
\hline Centaurium littorale spp. uliginosum & C. albicans & $0.2 \mathrm{mg} / \mathrm{mL}$ & Siler et al. (2014) \\
\hline Centaurium pulchellum & C. albicans & $0.4 \mathrm{mg} / \mathrm{mL}$ & Siler et al. (2014) \\
\hline Centaurium spicatum & C. albicans & $0.2 \mathrm{mg} / \mathrm{mL}$ & Siler et al. (2014) \\
\hline Centaurium tenuiflorum & C. albicans & $0.2 \mathrm{mg} / \mathrm{mL}$ & Siler et al. (2014) \\
\hline Crossandra infundibuliformis & C. krusei & $125 \mathrm{mg} / \mathrm{mL}$ & Madhumitha and Saral (2011) \\
\hline \multirow[t]{3}{*}{ Diospyros canaliculata } & C. albicans & $0.0125 \mathrm{mg} / \mathrm{mL}$ & Dzoyem et al. (2011) \\
\hline & C. kefyr & $0.025 \mathrm{mg} / \mathrm{mL}$ & \\
\hline & C. parapsilosis & $0.0125 \mathrm{mg} / \mathrm{mL}$ & \\
\hline \multirow[t]{2}{*}{ Dorstenia turbinata } & C. albicans & $0.039 \mathrm{mg} / \mathrm{mL}$ & Ngameni et al. (2009) \\
\hline & C. glabrata & $0.039 \mathrm{mg} / \mathrm{mL}$ & \\
\hline Ephedra pachyclada & C. albicans & $0.0005 \mathrm{mg} / \mathrm{mL}^{\mathrm{a}}$ & Parsaeimehr et al. (2010) \\
\hline Ephedra procera & C. albicans & $0.0005 \mathrm{mg} / \mathrm{mL}^{\mathrm{a}}$ & Parsaeimehr et al. (2010) \\
\hline Ephedra strobilacea & C. albicans & $0.001 \mathrm{mg} / \mathrm{mL}^{\mathrm{a}}$ & Parsaeimehr et al. (2010) \\
\hline \multirow[t]{3}{*}{ Equinops polyceras } & C. albicans & $6.3 \mathrm{mg} / \mathrm{mL}$ & Darwish and Aburjai \\
\hline & C. glabrata & $12.5 \mathrm{mg} / \mathrm{mL}$ & $(2011)$ \\
\hline & C. krusei & $3.1 \mathrm{mg} / \mathrm{mL}$ & \\
\hline Eucalyptus intertexta & C. albicans & $0.0625 \mathrm{mg} / \mathrm{mL}$ & Safaei-Ghomi and And (2010) \\
\hline Eucalyptus largiflorens & C. albicans & $0.0312 \mathrm{mg} / \mathrm{mL}$ & Safaei-Ghomi and Ahd (2010) \\
\hline Eucalyptus sideroxylon & C. albicans & $400 \mathrm{mg} / \mathrm{mL}^{*}$ & Ashour (2008) \\
\hline Eucalyptus torquata & C. albicans & $400 \mathrm{mg} / \mathrm{mL}^{*}$ & Ashour (2008) \\
\hline Euphorbia hirta & C. albicans & $3.13 \mathrm{mg} / \mathrm{mL}$ & Rajeh et al. (2010) \\
\hline \multirow[t]{6}{*}{ Euphorbia macroclada } & C. albicans & $12.5 \mathrm{mg} / \mathrm{mL}$ & Darwish and Aburjai \\
\hline & & $25 \mathrm{mg} / \mathrm{mL}$ & $(2011)$ \\
\hline & C. glabrata & $12.5 \mathrm{mg} / \mathrm{mL}$ & \\
\hline & & $25 \mathrm{mg} / \mathrm{mL}$ & \\
\hline & C. krusei & $12.5 \mathrm{mg} / \mathrm{mL}$ & \\
\hline & & $25 \mathrm{mg} / \mathrm{mL}$ & \\
\hline Euphorbia tirucalli & C. albicans & $0.125 \mathrm{mg} / \mathrm{disc}^{\mathrm{a}}$ & Parekh and Chanda (2008) \\
\hline Glycyrrhiza glabra & C. albicans & $0.0005 \mathrm{mg} / \mathrm{mL}^{\mathrm{a}}$ & Karomi et al. (2012) \\
\hline Gonzalagunia rosea & C. albicans & $1.25 \mathrm{mg} / \mathrm{mL}$ & Niño et al. (2007) \\
\hline Holarrhena antidysenterica & C. tropicalis & $0.125 \mathrm{mg} / \mathrm{disc}^{\mathrm{a}}$ & Parekh and Chanda (2008) \\
\hline Hybiscus sabdariffa & C. krusei & $25 \mathrm{mg} / \mathrm{mL}$ & Darwish and Aburjai (2011) \\
\hline Hypericum & C. albicans & $12.5 \mathrm{mg} / \mathrm{mL}$ & Darwish and Aburjai \\
\hline \multirow[t]{2}{*}{ trequetrifolium } & C. glabrata & $25 \mathrm{mg} / \mathrm{mL}$ & $(2011)$ \\
\hline & C. krusei & $12.5 \mathrm{mg} / \mathrm{mL}$ & \\
\hline Hyssopus officinalis subsp. pilifer & C. albicans & $10 \mathrm{mg} / \mathrm{mL}$ & Džamić et al. (2013) \\
\hline Laurus nobilis & C. albicans & $0.13 \mathrm{mg} / \mathrm{mL}$ & Unver et al. (2008) \\
\hline & C. glabrata & $0.13 \mathrm{mg} / \mathrm{mL}$ & \\
\hline & C. holmii & $0.16 \mathrm{mg} / \mathrm{mL}$ & \\
\hline & C. insane & $0.07 \mathrm{mg} / \mathrm{mL}$ & \\
\hline & C. krusei & $0.07 \mathrm{mg} / \mathrm{mL}$ & \\
\hline & C. tropicalis & $0.04 \mathrm{mg} / \mathrm{mL}$ & \\
\hline Leiothrix spiralis & C. albicans & $0.5 \mathrm{mg} / \mathrm{mL}$ & Araújo et al. (2012) \\
\hline & C. krusei & $0.5 \mathrm{mg} / \mathrm{mL}$ & \\
\hline & C. parapsilosis & $0.25 \mathrm{mg} / \mathrm{mL}$ & \\
\hline & C. tropicalis & $1 \mathrm{mg} / \mathrm{mL}$ & \\
\hline Lepidium sativum & C. albicans & $25 \mathrm{mg} / \mathrm{mL}$ & Darwish and Aburjai (2011) \\
\hline Mangifera indica & C. albicans & $0.125 \mathrm{mg} / \mathrm{disc}^{\mathrm{a}}$ & Parekh and Chanda (2008) \\
\hline Mentha longifolia & C. albicans & $6.3 \mathrm{mg} / \mathrm{mL}$ & Darwish and Aburjai \\
\hline & C. glabrata & $12.5 \mathrm{mg} / \mathrm{mL}$ & $(2011)$ \\
\hline & C. krusei & $12.5 \mathrm{mg} / \mathrm{mL}$ & \\
\hline Mesua ferra & C. albicans & $0.125 \mathrm{mg} / \mathrm{disc}^{\mathrm{a}}$ & Parekh and Chanda (2008) \\
\hline Mindium laevigatum & C. albicans & $0.1 \mathrm{mg} / \mathrm{mL}$ & Modaressi et al. (2013) \\
\hline Montanoa & C. albicans & $0.62 \mathrm{mg} / \mathrm{mL}$ & Niño et al. (2007) \\
\hline Munnozia senecionidis & C. albicans & $2.5 \mathrm{mg} / \mathrm{mL}$ & Niño et al. (2007) \\
\hline
\end{tabular}


Table 5 (Continued)

\begin{tabular}{|c|c|c|c|}
\hline Plant sources & Candida species & MIC & References \\
\hline \multirow[t]{3}{*}{ Origanum syriacum } & C. albicans & $6.3 \mathrm{mg} / \mathrm{mL}$ & Darwish and Aburjai \\
\hline & C. glabrata & $12.5 \mathrm{mg} / \mathrm{mL}$ & $(2011)$ \\
\hline & C. krusei & $12.5 \mathrm{mg} / \mathrm{mL}$ & \\
\hline Paeonia rockii & C. albicans & $0.025 \mathrm{mg} / \mathrm{mL}$ & Picerno et al. (2011) \\
\hline \multirow[t]{3}{*}{ Phlomis brachydon } & C. albicans & $6.3 \mathrm{mg} / \mathrm{mL}$ & Darwish and Aburjai \\
\hline & C. glabrata & $12.5 \mathrm{mg} / \mathrm{mL}$ & $(2011)$ \\
\hline & C. krusei & $3.1 \mathrm{mg} / \mathrm{mL}$ & \\
\hline \multirow[t]{3}{*}{ Pimpinella anisum } & C. albicans & $12.5 \mathrm{mg} / \mathrm{mL}$ & Darwish and Aburjai \\
\hline & C. glabrata & $12.5 \mathrm{mg} / \mathrm{mL}$ & $(2011)$ \\
\hline & C. krusei & $25 \mathrm{mg} / \mathrm{mL}$ & \\
\hline Pimpinella anisum & C. albicans & $16 \mathrm{mg} / \mathrm{mL}$ & Yazdani et al. (2009) \\
\hline Psidium sartorianum & C. albicans & $16 \mathrm{mg} / \mathrm{mL}$ & Camacho-Hernández et al. (2004) \\
\hline \multirow[t]{7}{*}{ Ribes $x$ nidigrolaria } & C. glabrata & $2.91 \mathrm{mg} / \mathrm{mL}$ & Krisch et al. (2008) \\
\hline & C. inconspicua & $3.33 \mathrm{mg} / \mathrm{mL}$ & \\
\hline & C. lipolytica & $4.67 \mathrm{mg} / \mathrm{mL}$ & \\
\hline & C. norvegica & $10.98 \mathrm{mg} / \mathrm{mL}$ & \\
\hline & C. parapsilosis & $3.56 \mathrm{mg} / \mathrm{mL}$ & \\
\hline & C. tropicalis & $4.23 \mathrm{mg} / \mathrm{mL}$ & \\
\hline & C. zeylanoides & $5.68 \mathrm{mg} / \mathrm{mL}$ & \\
\hline \multirow[t]{4}{*}{ Ribes nigrum } & C. guilliermondii & $6.13 \mathrm{mg} / \mathrm{mL}$ & Krisch et al. (2008) \\
\hline & C. inconspicua & $4.22 \mathrm{mg} / \mathrm{mL}$ & \\
\hline & C. parapsilosis & $4.41 \mathrm{mg} / \mathrm{mL}$ & \\
\hline & C. tropicalis & $7.16 \mathrm{mg} / \mathrm{mL}$ & \\
\hline \multirow{2}{*}{ Ribes uva-crispa } & C. glabrata & $4.60 \mathrm{mg} / \mathrm{mL}$ & Krisch et al. (2008) \\
\hline & C. lipolytica & $4.63 \mathrm{mg} / \mathrm{mL}$ & \\
\hline \multirow[t]{2}{*}{ Salvia officinalis } & C. clus & $0.16 \mathrm{mg} / \mathrm{mL}$ & Unver et al. (2008) \\
\hline & C. tropicalis & $0.16 \mathrm{mg} / \mathrm{mL}$ & \\
\hline Saussurea lappa & C. albicans & $0.125 \mathrm{mg} / \mathrm{disc}^{\mathrm{a}}$ & Parekh and Chanda (2008) \\
\hline Schistocarpha sinforosi & C. albicans & $2.5 \mathrm{mg} / \mathrm{mL}$ & Niño et al. (2007) \\
\hline Schizozygia caffaeoides & C. albicans & $10 \mathrm{mg} / \mathrm{mL}$ & Kariba et al. (2001) \\
\hline Sclerocarya birrea & C. parapsilosis & $0.32 \mathrm{mg} / \mathrm{mL}$ & Masoko et al. (2008) \\
\hline \multirow{3}{*}{ Thea sinensis } & C. albicans & $6.3 \mathrm{mg} / \mathrm{mL}$ & Darwish and Aburjai \\
\hline & C. glabrata & $12.5 \mathrm{mg} / \mathrm{mL}$ & $(2011)$ \\
\hline & C. krusei & $12.5 \mathrm{mg} / \mathrm{mL}$ & \\
\hline Thecacoris annobonae & C. albicans & $0.3125 \mathrm{mg} / \mathrm{mL}$ & Kuete et al. (2010) \\
\hline \multirow[t]{6}{*}{ Thymbra spicata } & C. albicans & $0.04 \mathrm{mg} / \mathrm{mL}$ & Unver et al. (2008) \\
\hline & C. clus & $0.01 \mathrm{mg} / \mathrm{mL}$ & \\
\hline & C. glabrata & $0.01 \mathrm{mg} / \mathrm{mL}$ & \\
\hline & C. krusei & $0.01 \mathrm{mg} / \mathrm{mL}$ & \\
\hline & C. parapsilosis & $0.01 \mathrm{mg} / \mathrm{mL}$ & \\
\hline & C. tropicalis & $0.01 \mathrm{mg} / \mathrm{mL}$ & \\
\hline Trapa natans & C. tropicalis & $0.125 \mathrm{mg} / \mathrm{disc}^{\mathrm{a}}$ & Parekh and Chanda (2008) \\
\hline Trigonella & C. albicans & $12.5 \mathrm{mg} / \mathrm{mL}$ & Darwish and Aburjai \\
\hline foenum-graecum & C. krusei & $12.5 \mathrm{mg} / \mathrm{mL}$ & $(2011)$ \\
\hline Varthemia iphionoides & C. albicans & $6.3 \mathrm{mg} / \mathrm{mL}$ & Darwish and Aburjai (2011) \\
\hline Verbesina nudipes & C. albicans & $2.5 \mathrm{mg} / \mathrm{mL}$ & Niño et al. (2007) \\
\hline Vitex negundo & C. albicans & $0.125 \mathrm{mg} / \mathrm{disc}^{\mathrm{a}}$ & Parekh and Chanda (2008) \\
\hline
\end{tabular}

MIC - minimum inhibitory concentration.

a Concentration used to evaluate antifungal activity by disc diffusion.

nisms of action such as interaction with the lipid bilayer of the cell membrane, which consequently affect the membrane permeability, respiratory chain and cellular energy production (Tintino et al., 2014), as well as the inhibition of the $\mathrm{H}^{+}$-ATPase (Kuete et al., 2010). Beyond that it is possible to distinguish the antifungal potential, between fungistatic and/or fungicidal. The majority of the studies reported fungistatic effects of plant extracts (Anibal et al., 2013; Barros et al., 2013a; Basile et al., 2013; Correa-Royero et al., 2010; Dzoyem et al., 2014; Kariba et al., 2001; Kim and Lee, 2012; Kurdelas et al., 2010; Lubian et al., 2010; Nordin et al., 2013; Pathak, 2012; Thirach et al., 2003; Tomczykowa et al., 2008; Tsuzuki et al., 2007), but there are also some reports evidencing fungicidal effects, most of them in a dose-dependent manner (Askun et al., 2008; Barros et al., 2013a; Dzoyem et al., 2014; Korukluoglu et al., 2008; Lubian et al., 2010; Nordin et al., 2013; Rajeh et al., 2010; Siler et al., 2014; Sule et al., 2012; Yazdani et al., 2009).

It is crucial to characterize the phytochemical composition of the plant extracts with antifungal activity, in order to identify the individual compounds responsible for this bioactivity, and to elucidate the main molecular targets in Candida species. Some studies have been performed in individual phenolic compounds (one of the most abundant molecules in polar extracts), as it will be discussed in the next section but, unfortunately, the majority of the studies report anti-Candida activity of crude extracts without any chemical characterization.

\subsection{The use of phenolic compounds against Candida species}

Phenolic acids, flavonoids and tannins are the most studied groups of phenolic compounds, comprising molecules with evidenced antifungal properties. These molecules have been isolated from different plant sources, as also commercially obtained as pure compounds.

A commercial sample of gallic acid presented higher antifungal potential against $C$. albicans than the same compound isolated from Lythrum salicaria, Paeonia rockii or Pelargonium reniforme subsp. reniforme (Table 8). The same behaviour was observed for commercial quercetin, in comparison to the one obtained from Buddleja salviifolia and Halimodendron halodendron (Table 9). On the other hand, commercial sample of salicylic acid presented lower antifungal potential than the same compound isolated from $\mathrm{Hal}$ imodendron halodendron (Table 8). It would be expected a similar 
Table 6

Activity of hydroalcoholic extracts against Candida species.

\begin{tabular}{|c|c|c|c|c|}
\hline & Plant source & Candida species & MIC & References \\
\hline Ethanol: water $(90: 10 \mathrm{v} / \mathrm{v})$ & Sapindus saponaria & C. parapsilosis & $0.4 \mathrm{mg} / \mathrm{mL}$ & Tsuzuki et al. (2007) \\
\hline Ethanol: water $(70: 30 \mathrm{v} / \mathrm{v})$ & Emblica officinalis & C. albicans & $0.1024 \mathrm{mg} / \mathrm{mL}$ & Tharkar et al. (2010) \\
\hline Ethanol: water $(70: 30 \mathrm{v} / \mathrm{v})$ & Glycyrrhiza glabra & C. albicans & $0.512 \mathrm{mg} / \mathrm{mL}$ & Tharkar et al. (2010) \\
\hline Ethanol: water $(70: 30 \mathrm{v} / \mathrm{v})$ & Momordica charantia & C. guilhermondii & $0.1 \mathrm{mg} / \mathrm{mL}^{\mathrm{a}}$ & Silva (2012) \\
\hline Ethanol: water (70:30 & Punica granatum & C. albicans & $0.1 \mathrm{mg} / \mathrm{mL}^{\mathrm{a}}$ & Silva (2012) \\
\hline \multirow[t]{4}{*}{$\mathrm{v} / \mathrm{v})$} & & C. guilhermondii & $0.1 \mathrm{mg} / \mathrm{mL}^{\mathrm{a}}$ & \\
\hline & & C. krusei & $0.1 \mathrm{mg} / \mathrm{mL}^{\mathrm{a}}$ & \\
\hline & & C. parapsilosis & $0.1 \mathrm{mg} / \mathrm{mL}^{\mathrm{a}}$ & \\
\hline & & C. tropicalis & $0.1 \mathrm{mg} / \mathrm{mL}^{\mathrm{a}}$ & \\
\hline Ethanol: water (70:30 & Syzygium aromaticum & C. guilhermondii & $0.05 \mathrm{mg} / \mathrm{mL}$ & Silva (2012) \\
\hline \multirow[t]{2}{*}{$\mathrm{v} / \mathrm{v})$} & & C. parapsilosis & $0.1 \mathrm{mg} / \mathrm{mL}^{\mathrm{a}}$ & \\
\hline & & C. tropicalis & $0.1 \mathrm{mg} / \mathrm{mL}^{\mathrm{a}}$ & \\
\hline Ethanol: water $(50: 50 \mathrm{v} / \mathrm{v})$ & Achyranthes aspera & C. albicans & $0.3 \mathrm{mg} / \mathrm{mL}$ & Mathur et al. (2011) \\
\hline Ethanol: water (50:50 & Punica granatum & C. albicans & $0.0039 \mathrm{mg} / \mathrm{mL}$ & Endo et al. (2010) \\
\hline $\mathrm{v} / \mathrm{v})$ & & C. parapsilosis & $0.0039 \mathrm{mg} / \mathrm{mL}$ & \\
\hline Methanol: water $(90: 10 \mathrm{v} / \mathrm{v})$ & Cassia fistula & C. albicans & $0.025 \mathrm{mg} / \mathrm{mL}$ & Bhalodia et al. (2012) \\
\hline Methanol: water & Castanea sativa & C. albicans & $0.625 \mathrm{mg} / \mathrm{mL}$ & Barros et al. (2013a) \\
\hline$(80: 20 \mathrm{v} / \mathrm{v})$ & & C. tropicalis & $0.625 \mathrm{mg} / \mathrm{mL}$ & \\
\hline Methanol: water $(80: 20 \mathrm{v} / \mathrm{v})$ & Cistus lanadifer & C. tropicalis & $0.625 \mathrm{mg} / \mathrm{mL}$ & Barros et al. (2013b) \\
\hline Methanol: water & Filipendula ulmaria & C. albicans & $0.625 \mathrm{mg} / \mathrm{mL}$ & Barros et al. (2013a) \\
\hline$(80: 20 \mathrm{v} / \mathrm{v})$ & & C. tropicalis & $0.155 \mathrm{mg} / \mathrm{mL}$ & \\
\hline Methanol: water & Rosa micrantha & C. albicans & $0.05 \mathrm{mg} / \mathrm{mL}$ & Barros et al. (2013a) \\
\hline$(80: 20 \mathrm{v} / \mathrm{v})$ & & C. tropicalis & $0.155 \mathrm{mg} / \mathrm{mL}$ & \\
\hline Methanol: water & Salvia officinalis & C. parapsilosis & $2.5 \mathrm{mg} / \mathrm{mL}$ & Martins et al. (2015) \\
\hline$(80: 20 \mathrm{v} / \mathrm{v})$ & & C. tropicalis & $1.25 \mathrm{mg} / \mathrm{mL}$ & \\
\hline
\end{tabular}

MIC - minimum inhibitory concentration.

a Concentration used to evaluate antifungal activity by disc diffusion.

bioactivity for the same compound, independently of its source. Therefore, the observed differences are probably related with the purity grade of the tested compounds, as well as with the vehicles/solvents used to test antifungal activities.

Table 8 shows the activity of phenolic acids (hydroxycinnamic acid derivatives, hydroxybenzoic acid derivatives and other related compounds) against Candida species. In general, hydroxycinnamic acid derivatives presented lower MICs against Candida species, than the hydroxybenzoic acid derivatives. The only exception was reported by Faria et al. (2011); however, in this case, it is necessary to take into account that the results referred to tested concentrations and not MIC values. Analyzing the results by each Candida species, in general, commercial compounds, mainly albicanyl caffeate $(\mathrm{MIC}=4 \mu \mathrm{g} / \mathrm{mL}$ ) presented the most pronounced antifungal effects against $C$. albicans. Two phenolic acid related compounds, dihydro- $N$-caffeoyltyramine and trans- $N$ caffeoyltyramine (MICs $=5 \mu \mathrm{g} / \mathrm{mL}$ ) obtained from Lycium chinense also evidenced a significant potential, followed by gallic acid isolated from Paeonia rockii $(30 \mu \mathrm{g} / \mathrm{mL})$ and gentisic, syringic, vanillic and 4-O- $\beta$-D-(6-O-gentisoylglucosyl) vanillic acids from Stenoloma chusanum $(50 \mu \mathrm{g} / \mathrm{mL})$. Regarding other Candida species, only Latte and Kolodziej (2000) report the antifungal activity of gallic acid from Pelargonium reniforme subsp. reniforme against C. glabrata (MIC $=62 \mu \mathrm{g} / \mathrm{mL}$ ) and C. krusei (MIC $=125 \mu \mathrm{g} / \mathrm{mL}$ ). To authors' knowledge there are only reports regarding commercial compounds against $C$. parapsilosis, $C$. tropicalis and $C$. lusitaniae. The antifungal activity of flavonoids against Candida species is presented in Table 9. Commercial compounds displayed slight higher antifungal effects than flavonoids isolated from plant sources. Among the commercial compounds, silibinin (MIC $=4 \mu \mathrm{g} / \mathrm{mL}$ ), a flavolignan, exerted the most pronounced antifungal activity, against $C$. albicans, followed by apigenin (a flavones), genistein (an isoflavone), naringin (a flavanone), quercitin (a flavonol) and silymarin (a flavolignan), which presented similar potential (MIC $=8 \mu \mathrm{g} / \mathrm{mL}$ ). Concerning to the flavonoids derived from plant origin, 2'-O-methyllabronisoflavone $(16-25 \mu \mathrm{g} / \mathrm{mL})$ and 9-

Table 7

Activity of other extracts against Candida species.

\begin{tabular}{|c|c|c|c|c|}
\hline Extract & Plant source & Candida species & MIC & References \\
\hline Dimethyl sulfoxide & Eleutherine plicata & C. albicans & $259 \mathrm{mg} / \mathrm{mL}$ & Menezes et al. (2009) \\
\hline \multirow[t]{2}{*}{ (DMSO) } & Psidium guajava & & $125 \mathrm{mg} / \mathrm{mL}$ & \\
\hline & Syzygium aromaticum & & $62.5 \mathrm{mg} / \mathrm{mL}$ & \\
\hline Diethyl ether & Origanum vulgare & C. albicans & $5 \mathrm{mg} / \mathrm{mL}$ & Ličina et al. (2013) \\
\hline \multirow[t]{5}{*}{ Ethyl acetate } & Crossandra infundibuliformis & C. krusei & $125 \mathrm{mg} / \mathrm{mL}$ & Madhumitha and Saral (2011) \\
\hline & Curcuma zedoaria & C. albicans & $12.5 \mathrm{mg} / \mathrm{mL}$ & Zhu et al. (2005) \\
\hline & & C. lusitaniae & $12.5 \mathrm{mg} / \mathrm{mL}$ & \\
\hline & Hyssopus officinalis subsp. Pilifer & C. albicans & $7.00 \mathrm{mg} / \mathrm{mL}$ & Džamić et al. (2013) \\
\hline & Origanum vulgare & C. albicans & $5 \mathrm{mg} / \mathrm{mL}$ & Ličina et al. (2013) \\
\hline \multirow[t]{6}{*}{ Tinctures } & Anacardium occidentale & C. albicans & $100 \mathrm{mg} / \mathrm{mL}$ & Cardoso et al. (2012) \\
\hline & & C. krusei & $100 \mathrm{mg} / \mathrm{mL}$ & \\
\hline & Malva sylvestris & C. albicans & $100 \mathrm{mg} / \mathrm{mL}$ & Cardoso et al. (2012) \\
\hline & & C. krusei & $25 \mathrm{mg} / \mathrm{mL}$ & \\
\hline & & C. tropicalis & $100 \mathrm{mg} / \mathrm{mL}$ & \\
\hline & Salvia officinalis & C. krusei & $100 \mathrm{mg} / \mathrm{mL}$ & Cardoso et al. (2012) \\
\hline
\end{tabular}

MIC - minimum inhibitory concentration. 
Table 8

Activity of phenolics acids against Candida species.

\begin{tabular}{|c|c|c|c|c|}
\hline Phenolic acids & Source & Candida species & MIC & References \\
\hline \multicolumn{5}{|l|}{ Hydroxycinnamic acid derivatives } \\
\hline \multirow[t]{2}{*}{ Caffeic acid } & \multirow[t]{2}{*}{ Commercial compound } & C. albicans & $8 \mu \mathrm{g} / \mathrm{mL}$ & \multirow[t]{2}{*}{ Ozçelik et al. (2011) } \\
\hline & & C. parapsilosis & $16 \mu \mathrm{g} / \mathrm{mL}$ & \\
\hline \multirow[t]{2}{*}{ Chlorogenic acid } & \multirow[t]{2}{*}{ Commercial compound } & C. albicans & $8 \mu \mathrm{g} / \mathrm{mL}$ & \multirow[t]{2}{*}{ Ozçelik et al. (2011) } \\
\hline & & C. parapsilosis & $16 \mu \mathrm{g} / \mathrm{mL}$ & \\
\hline m-coumaric acid (2-hydroxycinnamic acid) & Commercial compound & $\begin{array}{l}\text { C. albicans; C. parapsilosis; } \\
\text { C. glabrata; C. tropicalis; } C \text {. } \\
\text { krusei; C. lusitaniae }\end{array}$ & $820 \mu \mathrm{g} / \mathrm{mL}^{\mathrm{a}}$ & Faria et al. (2011) \\
\hline o-coumaric acid (3-hydroxycinnamic acid) & Commercial compound & $\begin{array}{l}\text { C. albicans; } C \text {. } \\
\text { parapsilosis; } \\
\text { C. glabrata; } C \text {. } \\
\text { tropicalis; } C \text {. } \\
\text { krusei; } C \text {. } \\
\text { lusitaniae }\end{array}$ & $820 \mu \mathrm{g} / \mathrm{mL}^{\mathrm{a}}$ & Faria et al. (2011) \\
\hline \multirow{3}{*}{$\begin{array}{l}p \text {-coumaric acid } \\
\text { (4-hydroxycinnamic } \\
\text { acid) }\end{array}$} & \multirow[t]{3}{*}{ Commercial compound } & C. albicans & $40 \mu \mathrm{g} / \mathrm{mL}$ & Siler et al. (2014) \\
\hline & & C. albicans; C. parapsilosis; & $820 \mu \mathrm{g} / \mathrm{mL}^{\mathrm{a}}$ & \multirow[t]{2}{*}{ Faria et al. (2011) } \\
\hline & & $\begin{array}{l}\text { C. glabrata; } \text { C. tropicalis; } C \text {. } \\
\text { krusei; C. lusitaniae }\end{array}$ & & \\
\hline Ferulic acid & Commercial compound & C. albicans & $20 \mu \mathrm{g} / \mathrm{mL}$ & Siler et al. (2014) \\
\hline 4-hydroxy-3-methoxy cinnamic acid & Halimodendron halodendron & C. albicans & $200 \mu \mathrm{g} / \mathrm{mL}$ & Wang et al. (2012) \\
\hline \multicolumn{5}{|l|}{ Hydroxybenzoic acid derivatives } \\
\hline \multirow[t]{7}{*}{ Gallic acid } & Lythrum salicaria & C. albicans & $2500 \mu \mathrm{g} / \mathrm{mL}$ & Manayi et al. (2013) \\
\hline & Paeonia rockii & C. albicans & $30 \mu \mathrm{g} / \mathrm{mL}$ & Picerno et al. (2011) \\
\hline & \multirow{3}{*}{$\begin{array}{l}\text { Pelargonium reniforme } \\
\text { subsp. reniforme }\end{array}$} & C. albicans & $500 \mu \mathrm{g} / \mathrm{mL}$ & \multirow{3}{*}{$\begin{array}{l}\text { Latte and Kolodziej } \\
\text { (2000) }\end{array}$} \\
\hline & & C. glabrata & $62 \mu \mathrm{g} / \mathrm{mL}$ & \\
\hline & & C. krusei & $125 \mu \mathrm{g} / \mathrm{mL}$ & \\
\hline & \multirow[t]{2}{*}{ Commercial compound } & C. albicans & $8 \mu \mathrm{g} / \mathrm{mL}$ & \multirow[t]{2}{*}{ Ozçelik et al. (2011) } \\
\hline & & C. parapsilosis & $16 \mu \mathrm{g} / \mathrm{mL}$ & \\
\hline Gentisic acid & Stenoloma chusanum & C. albicans & $50 \mu \mathrm{g} / \mathrm{mL}$ & Ren et al. (2009) \\
\hline $2-O-\beta-D-(6-O$-gentisoylglucosyl) gentisic acid & Stenoloma chusanum & C. albicans & $100 \mu \mathrm{g} / \mathrm{mL}$ & Ren et al. (2009) \\
\hline$p$-Hydroxybenzoic acid & Halimodendron halodendron & C. albicans & $100 \mu \mathrm{g} / \mathrm{mL}$ & Wang et al. (2012) \\
\hline \multirow[t]{2}{*}{ Salicylic acid } & Halimodendron halodendron & C. albicans & $150 \mu \mathrm{g} / \mathrm{mL}$ & Wang et al. (2012) \\
\hline & Commercial compound & $\begin{array}{l}\text { C. albicans; } C \text {. } \\
\text { parapsilosis; } C \text {. } \\
\text { glabrata; } C \text {. } \\
\text { tropicalis } \\
\text { C. krusei; } C . \\
\text { lusitaniae }\end{array}$ & $690 \mu \mathrm{g} / \mathrm{mL}^{\mathrm{a}}$ & Faria et al. (2011) \\
\hline Syringic acid & Stenoloma chusanum & C. albicans & $50 \mu \mathrm{g} / \mathrm{mL}$ & Ren et al. (2009) \\
\hline Vanillic acid & Stenoloma chusanum & C. albicans & $50 \mu \mathrm{g} / \mathrm{mL}$ & Ren et al. (2009) \\
\hline $4-O-\beta$-D-(6-O-gentisoylglucosyl) vanillic acid & Stenoloma chusanum & C. albicans & $50 \mu \mathrm{g} / \mathrm{mL}$ & Ren et al. (2009) \\
\hline 4-Acetoxy vanillic acid & Thecacoris annobonae & C. albicans & $156.25 \mu \mathrm{g} / \mathrm{mL}$ & Kuete et al. (2010) \\
\hline \multicolumn{5}{|l|}{ Other phenolic acids/related compounds } \\
\hline Albicanyl caffeate & Commercial compound & C. albicans & $4 \mu \mathrm{g} / \mathrm{mL}$ & Sun et al. (2004) \\
\hline Dihydro-N-caffeoyltyramine ${ }^{b}$ & Lycium chinense & C. albicans & $5 \mu \mathrm{g} / \mathrm{mL}$ & Lee et al. (2004) \\
\hline cis- $N$-caffeoyltyramine ${ }^{\mathrm{b}}$ & Lycium chinense & C. albicans & $40 \mu \mathrm{g} / \mathrm{mL}$ & Lee et al. (2004) \\
\hline trans- $N$-Feruloyloctopamine ${ }^{b}$ & Lycium chinense & C. albicans & $10 \mu \mathrm{g} / \mathrm{mL}$ & Lee et al. (2004) \\
\hline trans- $N$-Caffeoyltyramine ${ }^{b}$ & Lycium chinense & C. albicans & $5 \mu \mathrm{g} / \mathrm{mL}$ & Lee et al. (2004) \\
\hline 3,3',4-tri-Omethylellagic acid-4-O- $\beta$-D-glucopyranoside & Lythrum salicaria & C. albicans & $312 \mu \mathrm{g} / \mathrm{mL}$ & Manayi et al. (2013) \\
\hline \multirow[t]{2}{*}{ Quinic acid } & Commercial compound & C. albicans & $8 \mu \mathrm{g} / \mathrm{mL}$ & Ozçelik et al. (2011) \\
\hline & & C. parapsilosis & $16 \mu \mathrm{g} / \mathrm{mL}$ & \\
\hline
\end{tabular}

MIC - Minimum inhibitory concentration.

a Tested concentration and not MIC

b Phenolic amides.

O-methyl-4-hydroxyboeravinone B $(48 \mu \mathrm{g} / \mathrm{mL})$, two isoflavones isolated from Mirabilis jalapa, also presented a pronounced antifungal potential against $C$. albicans, followed by the flavonols kaempferol, myricetin, quercitin and rutin from Origanum vulgare subsp. gracile and Origanum acutidens (except quercetin and rutin). In general, flavonols (mainly represented by kaempferol, myricetin and quercetin), followed by flavanones and flavolignans, were the most effective antifungal flavonoids against Candida species. Once again, $C$. albicans was the main studied Candida species, being only tested commercial flavonoids against $C$. parapsilosis and compounds isolated from Origanum species against $C$. glabrata.

Table 10 shows the antifungal activity of hydrolyzable tannins, stilbenes and xanthones against Candida species. Comparing the different hydrolyzable tannins isolated from Pelargonium reniforme subsp. reniforme, it is possible to conclude that similar antifungal effects were achieved; the obtained MIC values were the same for corilagin, glucogallin, methyl ester, pelargoniin and phyllantusiin, against $C$. albicans, $C$. glabrata and C. krusei. Comparing Pelargonium reniforme subsp. reniforme with Punica granatum, the hydrolyzable tannin, punicalagin, exerted higher antifungal effect against $C$. albicans ( $\sim 128$ times higher). Regarding stilbenes, resveratrol and 3,4'-difluorostilbene gave similar MIC values against $C$. albicans (Sun et al., 2004), while for xanthones the commercial compound mangiferin presented a more pronounced effect against C. albicans. 8-Carboxy-methyl-1,3,5,6-tetrahydroxyxanthone gave significantly different MIC values for each Candida species; $C$. krusei and $C$. parapsilosis were the most sensible to the mentioned compound $(15.7 \mu \mathrm{g} / \mathrm{mL})$, while $C$. albicans showed higher resistance $(62.5 \mu \mathrm{g} / \mathrm{mL})$. Among tannins, stilbenes and xanthones, the latter seem to be the most efficient, exhibiting lower MICs against Candida species. Overall, commercial compounds seem to be more efficient than phenolic compounds isolated from natu- 
Table 9

Activity of flavonoids against Candida species.

\begin{tabular}{|c|c|c|c|c|}
\hline Flavonoids & Source & Candida species & MIC & References \\
\hline \multicolumn{5}{|l|}{ Flavonols } \\
\hline Kaempferol & $\begin{array}{l}\text { Origanum vulgare subsp. gracile; } \\
\text { Origanum acutidens }\end{array}$ & C. albicans; C. glabrata & $\begin{array}{l}0.1 \mu \mathrm{g} / \mathrm{g}^{\mathrm{a}} \\
0.5 \mu \mathrm{g} / \mathrm{g}^{\mathrm{a}}\end{array}$ & Kursat et al. (2011) \\
\hline Morin & $\begin{array}{l}\text { Origanum vulgare subsp. gracile; } \\
\text { Origanum acutidens }\end{array}$ & C. albicans; C. glabrata & $\begin{array}{l}42.8 \mu \mathrm{g} / \mathrm{g}^{\mathrm{a}} \\
4.5 \mu \mathrm{g} / \mathrm{g}^{\mathrm{a}}\end{array}$ & Kursat et al. (2011) \\
\hline Myricetin & $\begin{array}{l}\text { Origanum vulgare subsp. gracile; } \\
\text { Origanum acutidens }\end{array}$ & C. albicans; C. glabrata & $\begin{array}{l}0.2 \mu \mathrm{g} / \mathrm{g}^{\mathrm{a}} \\
1.2 \mu \mathrm{g} / \mathrm{g}^{\mathrm{a}}\end{array}$ & Kursat et al. (2011) \\
\hline \multirow[t]{4}{*}{ Quercetin } & Buddleja salviifolia & C. albicans & $125 \mu \mathrm{g} / \mathrm{mL}$ & Pendota et al. (2013) \\
\hline & Origanum vulgare subsp. gracile & C. albicans; C. glabrata & $0.3 \mu \mathrm{g} / \mathrm{g}^{\mathrm{a}}$ & Kursat et al. (2011) \\
\hline & Halimodendron halodendron & C. albicans & $250 \mu \mathrm{g} / \mathrm{mL}$ & Wang et al. (2012) \\
\hline & Commercial compound & C. albicans; C. parapsilosis & $8 \mu \mathrm{g} / \mathrm{mL}$ & Ozçelik et al. (2011) \\
\hline 3-O-methylquercetin & Halimodendron halodendron & C. albicans & $200 \mu \mathrm{g} / \mathrm{mL}$ & Wang et al. (2012) \\
\hline 3,3'-di-O-methylquercetin & Halimodendron halodendron & C. albicans & $200 \mu \mathrm{g} / \mathrm{mL}$ & Wang et al. (2012) \\
\hline 8-O-methylretusin & Halimodendron halodendron & C. albicans & $250 \mu \mathrm{g} / \mathrm{mL}$ & Wang et al. (2012) \\
\hline 8-O-methylretusin-7-O- $\beta$-D-glucopyranoside & Halimodendron halodendron & C. albicans & $250 \mu \mathrm{g} / \mathrm{mL}$ & Wang et al. (2012) \\
\hline \multirow[t]{2}{*}{ Rutin } & Origanum vulgare subsp. gracile & C. albicans; C. glabrata & $6.4 \mu \mathrm{g} / \mathrm{g}^{\mathrm{a}}$ & Kursat et al. (2011) \\
\hline & Commercial compound & C. albicans & $40 \mu \mathrm{g} / \mathrm{mL}$ & Siler et al. (2014) \\
\hline \multicolumn{5}{|l|}{ Flavones } \\
\hline Apigenin & Commercial compound & $\begin{array}{l}\text { C. albicans; } \\
\text { C. parapsilosis }\end{array}$ & $\begin{array}{l}8 \mu \mathrm{g} / \mathrm{mL} \\
16 \mu \mathrm{g} / \mathrm{mL}\end{array}$ & Ozçelik et al. (2011) \\
\hline Luteolin-6-C- $\beta$-D-glucopyranoside & Leiothrix spiralis & C. parapsilosis & $125 \mu \mathrm{g} / \mathrm{mL}$ & (Araújo et al., 2011) \\
\hline 4'-methoxyluteolin-6-C- $\beta$-D-glucopyranoside & Leiothrix spiralis & C. albicans & $125 \mu \mathrm{g} / \mathrm{mL}$ & (Araújo et al., 2011) \\
\hline 3,5,7,8,4'-pentahydroxy-3'-methoxy flavone & Halimodendron halodendron & C. albicans & $250 \mu \mathrm{g} / \mathrm{mL}$ & \\
\hline \multicolumn{5}{|l|}{ Flavanones } \\
\hline Naringin & Commercial compound & $\begin{array}{l}\text { C. albicans } \\
\text { C. parapsilosis }\end{array}$ & $\begin{array}{l}8 \mu \mathrm{g} / \mathrm{mL} \\
16 \mu \mathrm{g} / \mathrm{mL}\end{array}$ & Ozçelik et al. (2011) \\
\hline $\begin{array}{l}\text { Naringenin } \\
\text { Isoflavones }\end{array}$ & Origanum acutidens & C. albicans; C. glabrata & $10.3 \mu \mathrm{g} / \mathrm{g}^{\mathrm{a}}$ & Kursat et al. (2011) \\
\hline Genistein & Commercial compound & C. albicans; C. parapsilosis & $8 \mu \mathrm{g} / \mathrm{mL}$ & Ozçelik et al. (2011) \\
\hline 2'-O-methylabronisoflavone & Mirabilis jalapa & C. albicans & $16-25 \mu \mathrm{g} / \mathrm{mL}$ & Yang et al. (2001) \\
\hline $\begin{array}{l}\text { 9-O-methyl-4-hydroxyboeravinone B } \\
\text { Flavolignan }\end{array}$ & Mirabilis jalapa & C. albicans & $48 \mu \mathrm{g} / \mathrm{mL}$ & Yang et al. (2001) \\
\hline Silibinin & Commercial compound & $\begin{array}{l}\text { C. albicans } \\
\text { C. parapsilosis }\end{array}$ & $\begin{array}{l}4 \mu \mathrm{g} / \mathrm{mL} \\
8 \mu \mathrm{g} / \mathrm{mL}\end{array}$ & Ozçelik et al. (2011) \\
\hline Silymarin & Commercial compound & $\begin{array}{l}\text { C. albicans } \\
\text { C. parapsilosis }\end{array}$ & $\begin{array}{l}8 \mu \mathrm{g} / \mathrm{mL} \\
16 \mu \mathrm{g} / \mathrm{mL}\end{array}$ & Ozçelik et al. (2011) \\
\hline
\end{tabular}

MIC - minimum inhibitory concentration.

a Compound concentration in the tested extract.

Table 10

Activity of hydrolyzable tannins, stilbenes and xanthones against Candida species.

\begin{tabular}{|c|c|c|c|c|}
\hline Hydrolyzable tannins & Source & Candida species & MIC & References \\
\hline Corilagin & $\begin{array}{l}\text { Pelargonium reniforme } \\
\text { subsp. reniforme }\end{array}$ & $\begin{array}{l}\text { C. albicans } \\
\text { C. glabrata } \\
\text { C. krusei }\end{array}$ & $\begin{array}{l}500 \mu \mathrm{g} / \mathrm{mL} \\
31 \mu \mathrm{g} / \mathrm{mL} \\
125 \mu \mathrm{g} / \mathrm{mL}\end{array}$ & $\begin{array}{l}\text { Latte and Kolodziej } \\
\text { (2000) }\end{array}$ \\
\hline Glucogallin & $\begin{array}{l}\text { Pelargonium reniforme } \\
\text { subsp. reniforme }\end{array}$ & $\begin{array}{l}\text { C. glabrata } \\
\text { C. krusei }\end{array}$ & $\begin{array}{l}31 \mu \mathrm{g} / \mathrm{mL} \\
125 \mu \mathrm{g} / \mathrm{mL}\end{array}$ & $\begin{array}{l}\text { Latte and Kolodziej } \\
\text { (2000) }\end{array}$ \\
\hline Methyl ester & $\begin{array}{l}\text { Pelargonium reniforme } \\
\text { subsp. reniforme }\end{array}$ & $\begin{array}{l}\text { C. glabrata } \\
\text { C. krusei }\end{array}$ & $\begin{array}{l}31 \mu \mathrm{g} / \mathrm{mL} \\
125 \mu \mathrm{g} / \mathrm{mL}\end{array}$ & $\begin{array}{l}\text { Latte and Kolodziej } \\
\text { (2000) }\end{array}$ \\
\hline Pelargoniin & $\begin{array}{l}\text { Pelargonium reniforme } \\
\text { subsp. reniforme }\end{array}$ & $\begin{array}{l}\text { C. albicans } \\
\text { C. glabrata } \\
\text { C. krusei }\end{array}$ & $\begin{array}{l}500 \mu \mathrm{g} / \mathrm{mL} \\
31 \mu \mathrm{g} / \mathrm{mL} \\
125 \mu \mathrm{g} / \mathrm{mL}\end{array}$ & $\begin{array}{l}\text { Latte and Kolodziej } \\
\text { (2000) }\end{array}$ \\
\hline Phyllantusiin & $\begin{array}{l}\text { Pelargonium reniforme } \\
\text { subsp. reniforme }\end{array}$ & $\begin{array}{l}\text { C. albicans } \\
\text { C. glabrata } \\
\text { C. krusei }\end{array}$ & $\begin{array}{l}500 \mu \mathrm{g} / \mathrm{mL} \\
31 \mu \mathrm{g} / \mathrm{mL} \\
125 \mu \mathrm{g} / \mathrm{mL}\end{array}$ & $\begin{array}{l}\text { Latte and Kolodziej } \\
\text { (2000) }\end{array}$ \\
\hline Punicalagin & Punica granatum & $\begin{array}{l}\text { C. albicans } \\
\text { C. parapsilosis }\end{array}$ & $\begin{array}{l}3.9 \mu \mathrm{g} / \mathrm{mL} \\
1.9 \mu \mathrm{g} / \mathrm{mL}\end{array}$ & Endo et al. (2010) \\
\hline Not specified & Rhizophora apiculate & C. albicans & $6250 \mu \mathrm{g} / \mathrm{mL}$ & Lim et al. (2006) \\
\hline Stilbenes & & & & \\
\hline Resveratrol & Commercial compound & $\begin{array}{l}\text { C. albicans } \\
\text { C. albicans }\end{array}$ & $\begin{array}{l}16 \mu \mathrm{g} / \mathrm{mL} \\
20 \mu \mathrm{g} / \mathrm{mL}\end{array}$ & $\begin{array}{l}\text { Sun et al. (2004) } \\
\text { Jung et al. (2005) }\end{array}$ \\
\hline 3,4'-difluorostilbene & Commercial compound & C. albicans & $16 \mu \mathrm{g} / \mathrm{mL}$ & Sun et al. (2004) \\
\hline Xanthones & & & & \\
\hline $\begin{array}{l}\text { 8-carboxy-methyl- } \\
\text { 1,3,5,6- } \\
\text { tetrahydroxyxanthone }\end{array}$ & Leiothrix spiralis & $\begin{array}{l}\text { C. albicans } \\
\text { C. krusei } \\
\text { C. parapsilosis } \\
\text { C. tropicalis }\end{array}$ & $\begin{array}{l}62.5 \mu \mathrm{g} / \mathrm{mL} \\
15.7 \mu \mathrm{g} / \mathrm{mL} \\
15.7 \mu \mathrm{g} / \mathrm{mL} \\
31.25 \mu \mathrm{g} / \mathrm{mL}\end{array}$ & Araújo et al. (2011) \\
\hline Mangiferin & Commercial compound & C. albicans & $40 \mu \mathrm{g} / \mathrm{mL}$ & Siler et al. (2014) \\
\hline
\end{tabular}

MIC - minimum inhibitory concentration. 
ral matrices probably due to their higher purity grade. Regarding phenolic compounds from natural sources, punicalagin (isolated from Punica granatum), followed by dihydro- $N$-caffeoyltyramine and trans- $N$-caffeoyltyramine (isolated from Lycium chinense) were the most efficient against $C$. albicans. The hydrolyzable tannins, carolagin, glucogallin, methyl ester, pelargonin and phyllantusiin (isolated from Pelargonium reniforme subsp. reniforme) were the most efficient for C. glabrata, while 8-carboxy-methyl-1,3,5,6tetrahydroxyxanthone, a xanthone isolated from Leiothrix spiralis was the most effective against C. krusei and C. tropicalis. Lastly, for C. parapsilosis, punicalagin (isolated from Punica granatum), followed by 8-carboxy-methyl-1,3,5,6-tetrahydroxyxanthone (isolated from Leiothrix spiralis) presented the most pronounced effects.

\section{Conclusions}

Being part of the commensal flora, Candida species are also related to several situations of overgrowth, and an increasing resistance to the current antifungal drugs has been observed. Despite the efforts in order to discover and/or synthetize new chemical molecules, more efficient and effective than the existents, it is necessary to consider that these compounds could be associated to a wide variety of side effects. Beyond that those experiments are very expensive and take a long time until the accreditation/commercialization of the products. Medicinal plants are used since ancient times and present several bioactive properties, due to their richness in some biomolecules, such as phenolic compounds. Several studies have been developed towards investigating the antifungal properties of phenolic compounds enriched extracts, against Candida species. For that, different extraction solvents have been used, namely, water, ethanol, methanol, methanoldichloromethane and even mixtures of the previous. In parallel, and due to the high antifungal potential evidenced by some plant extracts, an increasing number of studies have been evaluating the antifungal potential of phenolic compounds isolated from those matrices.

Despite the existence of a tenuous variation on the antifungal activity according to Candida species, in general, the plant extracts obtained using methanol, water and mixtures of the previous (hydroalcoholic extracts) were more effective against Candida species than the extracts prepared using a unique solvent. This higher antifungal activity might be related with the solubility of the active principles in the solvents used; phenolic compounds are one of the most abundant bioactive molecules present in those polar extracts. Hydrolysable tannins, followed by some phenolic amides and flavonoids seem to exert the most pronounced antiCandida effects. There are some evidences that the mentioned extracts/compounds affect fungal cells through interaction with the lipid bilayer of the cell membrane. They could act by disrupting the membrane integrity or by intercalation into cell wall and/or DNA. Nevertheless, much more studies are necessary not only to evaluate the antifungal potential of the reviewed and other medicinal plants, but also to establish clearly mechanisms of action involved in potential of phenolic compounds from plant origin against Candida species, as also validate the results by in vivo studies.

\section{Acknowledgements}

The authors are grateful to Foundation for Science and Technology (FCT, Portugal) for N. Martins grant (SFRH/BD/87658/2012), L. Barros researcher contract under "Programa Compromisso com Ciência - 2008" and financial support to the Research Centre CIMO (strategic project PEst-OE/AGR/UI0690/2011). This work was also supported by the Programa Operacional, Fatores de competitividade - COMPETE and by national funds through FCT - Fundação para a Ciência e a Tecnologia on the scope of the projects FCT
PTDC/SAU-MIC/119069/2010, RECI/EBB-EBI/0179/2012 and PEstOE/EQB/LA0023/2013. The authors thank the Project "BioHealth Biotechnology and Bioengineering approaches to improve health quality", Ref. NORTE-07-0124-FEDER-000027, co-funded by the Programa Operacional Regional do Norte (ON.2-O Novo Norte), QREN, FEDER.

\section{References}

Abdul, B.A., Hassan, A.M., Hassan, A.S., 2012. In vitro antimicrobial activity of Thymus vulgaris, Origanum vulgare and Rosmarinus officinalis against denta caries pathogens. Haitham J. Pure Appl. Sci. 25, 1-7.

Abi-Said, D., Anaissie, E., Uzun, O., Raad, I., Pinzcowski, H., Vartivarian, S., 1997. The epidemiology of hematogenous candidiasis caused by different Candida species. Clin. Infect. Dis. 24, 1122-1128.

Adekunle, A.A., Ikumapayi, A.M., 2006. Antifungal property and phytochemical screening of the crude extracts of Funtumia elastica and Mallotus oppositifolius. West Indian Med. J. 55, 9-13.

Agarwal, V., Lal, P., Pruthi, V., 2010. Effect of plant oils on Candida albicans. J. Microbiol. Immunol. Infect. 43, 447-451.

Ali-Shtayeh, M.S., Ghdeib, S.I.A., 1999. Antifungal activity of plant extracts against dermatophytes. Mycoses 42, 665-672.

Alves, M.J., Ferreira, I.C.F.R., Dias, J., Teixeira, V., Martins, A., Pintado, M., 2013. A review on antifungal activity of mushroom (basidiomycetes) extracts and isolated compounds. Curr. Top. Med. Chem. 13, 2648-2659.

Alves-Silva, J.M., Santos, S.M.D., Pintado, M.E., Pérez-Álvarez, J.A., Fernández-López, J., Viuda-Martos, M., 2013. Chemical composition and in vitro antimicrobial, antifungal and antioxidant properties of essential oils obtained from some herbs widely used in portugal. Food Control 32, 371-378.

Amara, A.A., Shibl, A., 2013. Role of probiotics in health improvement, infection control and disease treatment and management. Saudi Pharm. J., 1-8.

Anibal, P.C., Peixoto, I.T.A., Foglio, M.A., Höfling, J.F., 2013. Antifungal activity of the ethanolic extracts of Punica granatum L. and evaluation of the morphological and structural modifications of its compounds upon the cells of Candida spp. Braz. J. Microbiol. 44, 839-848.

Araújo, M.G.F., Hilário, F., Vilegas, W., Santos, L.C., Brunetti, I.L., Sotomayor, C.E., Bauab, T.M., 2012. Correlation among antioxidant, antimicrobial, hemolytic, and antiproliferative properties of Leiothrix spiralis leaves extract. Int. J. Mol. Sci. 13, 9260-9277.

Araújo, M.G.D.F., Hilário, F., Nogueira, L.G., Vilegas, W., Santos, L.C., Bauab, T.M., 2011. Chemical constituents of the methanolic extract of leaves of Leiothrix spiralis ruhland and their antimicrobial activity. Molecules 16, 10479-10490.

Arif, T., Bhosale, J.D., Kumar, N., Mandal, T.K., Bendre, R.S., Lavekar, G.S., Dabur, R., 2009. Natural products - antifungal agents derived from plants. J. Asian Nat. Prod. Res. 11, 621-638.

Arif, T., Mandal, T.K., Dabur, R., 2011. Natural Products: Anti-fungal Agents Derived from Plants, Opportunity, Challenge and Scope of Natural Products in Medicinal Chemistry. pp. 283-311.

Asgarpanah, J., Kazemivash, N., 2012. Phytochemistry, pharmacology and medicinal properties of Coriandrum sativum L. Afr. J. Pharm. Pharmacol. 6, 2340-2345.

Ashour, H.M., 2008. Antibacterial, antifungal, and anticancer activities of volatile oils and extracts from stems, leaves, and flowers of Eucalyptus sideroxylon and Eucalyptus torquata. Cancer Biol. Ther. 7, 399-403.

Askun, T., Tumen, G., Satil, F., Kilic, T., 2008. Effects of some Lamiaceae species methanol extracts on potential mycotoxin producer fungi. Pharm. Biol. 46 688-694.

Asl, M.N., Hosseinzadeh, H., 2008. Review of pharmacological effects of Glycyrrhiza sp. and its bioactive compounds. Phyther. Res. 22, 709-724.

Asmundsdóttir, L.R., Erlendsdóttir, H., Agnarsson, B.A., Gottfredsson, M., 2009. The importance of strain variation in virulence of Candida dubliniensis and Candida albicans: results of a blinded histopathological study of invasive candidiasis. Clin. Microbiol. Infect. 15, 576-585.

Bakkali, F., Averbeck, S., Averbeck, D., Idaomar, M., 2008. Biological effects of essential oils - a review. Food Chem. Toxicol. 46, 446-475.

Balch, P.A., 2006. Prescription For Nutritional Healing, Fourth ed. Penguin Group, London, England.

Barros, L., Alves, C.T., Dueñas, M., Silva, S., Oliveira, R., Carvalho, A.M., Henriques, M., Santos-Buelga, C., Ferreira, I.C.F.R., 2013a. Characterization of phenolic compounds in wild medicinal flowers from portugal by HPLC-DAD-ESI/MS and evaluation of antifungal properties. Ind. Crops Prod. 44, 104-110.

Barros, L., Dueñas, M., Alves, C.T., Silva, S., Henriques, M., Santos-Buelga, C., Ferreira, I.C.F.R., 2013b. Antifungal activity and detailed chemical characterization of Cistus ladanifer phenolic extracts. Ind. Crops Prod. 41, 41-45.

Basile, A., Rigano, D., Conte, B., Bruno, M., Rosselli, S., Sorbo, S., 2013. Antibacterial and antifungal activities of acetonic extract from Paullinia cupana Mart. Seeds. Nat. Prod. Res. 27, 2084-2090.

Bhalodia, N.R., Nariya, P.B., Acharya, R.N., Shukla, V.J., 2012. In vitro antibacterial and antifungal activities of Cassia fistula Linn. fruit pulp extracts. AYU 33, $123-129$.

Blanco, J.L., Garcia, M.E., 2008. Immune response to fungal infections. Vet. Immunol. Immunopathol. 125, 47-70.

Brunke, S., Hube, B., 2013. Two unlike cousins: Candida albicans and C. Glabrata infection strategies. Cell. Microbiol. 15, 701-708. 
Camacho-Hernández, I.L., Cisneros-Rodríguez, C., Uribe-Beltrán, M.J., Ríos-Morgan, A., Delgado-Vargas, F., 2004. Antifungal activity of fruit pulp extract from Psidium sartorianum. Fitoterapia 75, 401-404.

Campos, L., 2008. Entender a bioquimica, 5th ed.

Cardoso, A.M.R., Cavalcanti, Y.W., Almeida, L.F.D., Pérez, A.L.A.L., Padilha, W.W.N., 2012. Antifungal activity of plant-based tinctures on Candida. RSBO 9, 25-30.

Carocho, M., Ferreira, I.C.F.R. 2013a. A review on antioxidants, prooxidants and related controversy: natural and synthetic compounds, screening and analysis methodologies and future perspectives. Food Chem. Toxicol. 51, 15-25.

Carocho, M., Ferreira, I.C.F.R., 2013b. The role of phenolic compounds in the fight against cancer - a review. Anticancer Agents Med. Chem. 13, 1236-1258.

Carrara, M.A., Donatti, L., Damke, E., Svidizinski, T.I.E., Consolaro, M.E.L., Batista, M.R., 2010. A new model of vaginal infection by Candida albicans in rats. Mycopathologia 170, 331-338.

Çekiç, S.D., Cetinkaya, A., Avan, A.N., Apak, R., 2013. Correlation of total antioxidant capacity with reactive oxygen species (ROS) consumption measured by oxidative conversion. J. Agric. Food Chem. 61, 5260-5270.

Chaturvedi, R.K., Beal, M.F., 2013. Mitochondrial diseases of the brain. Free Radic. Biol. Med. 63, 1-29.

Choudhary, M.I., Atta-ur-Rahmant, 1999. Recent studies on bioactive natural products. Pure Appl. Chem. 71, 1079-1081.

Coopoosamy, R.M., Magwa, M.L., 2007. Traditional use, antibacterial activity and antifungal activity of crude extract of Aloe excelsa. Afr. J. Biotechnol. 6 2406-2410.

Correa-Royero, J., Tangarife, V., Durán, C., Stashenko, E., Mesa-Arango, A., 2010. In vitro antifungal activity and cytotoxic effect of essential oils and extracts of medicinal and aromatic plants against Candida krusei and Aspergillus fumigatus. Braz. J. Pharmacogn. 20, 734-741.

Dai, J., Mumper, R.J., 2010. Plant phenolics: extraction, analysis and their antioxidant and anticancer properties. Molecules 15, 7313-7352.

Darwish, R.M., Aburjai, T.A., 2011. Antimicrobial activity of some medicinal plants against different Candida species. Jordan J. Pharm. Sci. 4, 70-80.

Devasagayam, T.P.A., Tilak, J.C., Boloor, K.K., Sane, K.S., Ghaskadbi, S.S., Lele, R.D. 2004. Free radicals and antioxidants in human health: current status and future prospects. J. Assoc. Phys. India 52, 794-804.

Develoux, M., Bretagne, S., 2005. Candidoses et levuroses diverses. EMC - Mal. Infect. 2, 119-139.

Dib, M.A., Djabou, N., Allali, H., Tabti, B., 2010. Identification of phenolic compounds and antimicrobial activity of roots of Arbutus unedo L. Asian J. Chem. 22, 4045-4053.

Doddanna, S.J., Patel, S., Sundarrao, M.A., Veerabhadrappa, R.S., 2013. Antimicrobia activity of plant extracts on Candida albicans: an in vitro study. Indian J. Dent. Res. 24, 401-405.

Džamić, A.M., Soković, M.D., Novaković, M., Jadranin, M., Ristić, M.S., Tešević, V. Marin, P.D., 2013. Composition, antifungal and antioxidant properties of Hyssopus officinalis L. subsp. pilifer (Pant.) Murb. essential oil and deodorized extracts. Ind. Crops Prod. 51, 401-407.

Dzoyem, J.P., Kechia, F.A., Kuete, V., Pieme, A.C., Akak, C.M., Tangmouo, J.G., Lohoue P.J., 2011. Phytotoxic, antifungal activities and acute toxicity studies of the crude extract and compounds from Diospyros canaliculata. Nat. Prod. Res. 25 $741-749$

Dzoyem, J.P., Tangmouo, J.G., Lontsi, D., Etoa, F.X., Lohoue, P.J., 2007. In vitro antifungal activity of extract and plumbagin from the stem bark of Diospyros crassiflora Hiern (Ebenaceae). Phyther. Res. 21, 671-674

Dzoyem, J.P., Tchuenguem, R.T., Kuiate, J.R., Teke, G.N., Kechia, F.A., Kuete, V., 2014 In vitro and in vivo antifungal activities of selected Cameroonian dietary spices. BMC Complement. Altern. Med 14 (1), 8.

Eggimann, P., Garbino, J., Pittet, D., 2003a. Epidemiology of Candida species infections in critically ill non-immunosuppressed patients. Lancet Infect. Dis. 3, 685-702.

Eggimann, P., Garbino, J., Pittet, D., 2003b. Management of Candida species infections in critically ill patients. Lancet Infect. Dis. 3, 772-785.

Endo, E.H., Cortez, D.A.G., Ueda-Nakamura, T., Nakamura, C.V., Filho, B.P.D., 2010. Potent antifungal activity of extracts and pure compound isolated from pomegranate peels and synergism with fluconazole against Candida albicans. Res. Microbiol. 161, 534-540.

Epstein, J.B., Polsky, B., 1998. Oropharyngeal candidiasis: a review of its clinical spectrum and current therapies. Clin. Ther. 20, 40-57.

Ertürk, Ö., 2006. Antibacterial and antifungal activity of ethanolic extracts from eleven spice plants. Biologia (Bratisl) 61, 275-278

Ettefagh, K.A., Burns, J.T., Junio, H.A., Kaatz, G.W., Cech, N.B., 2011. Goldenseal (Hydrastis canadensis L.) extracts synergistically enhance the antibacterial activity of berberine via efflux pump inhibition. Planta Med. 77, 835-840.

Fanello, S., Bouchara, J.P., Jousset, N., Delbos, V., LeFlohic, A.M., 2001. Nosocomia Candida albicans acquisition in a geriatric unit: epidemiology and evidence for person-to-person transmission. J. Hosp. Infect. 47, 46-52.

Farhat, M., Ben, Jordán, M.J., Chaouech-Hamada, R., Landoulsi, A., Sotomayor, J.A., 2009. Variations in essential oil, phenolic compounds, and antioxidant activity of tunisian cultivated Salvia officinalis L. J. Agric. Food Chem. 57, 10349-10356.

Farhat, M., Ben, Landoulsi, A., Chaouch-Hamada, R., Sotomayor, J.A., Jordán, M.J. 2013. Characterization and quantification of phenolic compounds and antioxidant properties of Salvia species growing in different habitats. Ind. Crops Prod. 49, 904-914

Faria, N.C.G., Kim, J.H., Gonçalves, L.A.P., Martins, M. de L., Chan, K.L., Campbell, B.C., 2011. Enhanced activity of antifungal drugs using natural phenolics against yeast strains of Candida and Cryptococcus. Lett. Appl. Microbiol. 52, 506-513.
Fatima, A., Gupta, V.K., Luqman, S., Negi, A.S., Kumar, J.K., Shanker, K., Saikia, D., Srivastava, S., Darokar, M.P., Khanuja, S.P.S., 2009. Antifungal activity of Glycyrrhiza glabra extracts and its active constituent glabridin. Phyther. Res. 1193, 1190-1193.

Fei, L.C., Chen, P.Y., Ahmad, Z., Othman, F., Pei Pei, C., 2008. Antifungal properties of Allium sativum extracts on Candida species. J. Trop. Med. Plants 9 , 43-48.

Franz, R., Kelly, S.L., Lamb, D.C., Kelly, D.E., Ruhnke, M., Morschhäuser, J., 1998. Multiple molecular mechanisms contribute to a stepwise development of fluconazole resistance in clinical Candida albicans strains. Antimicrob. Agents Chemother, 42, 3065-3072.

Geiger, A.M., Foxman, B., Sobel, J.D., 1995. Chronic vulvovaginal candidiasis: characteristics of women with Candida albicans, C. Glabrata and no Candida. Genitourin. Med. 71, 304-307.

Ghannoum, M.A., Rice, L.B., 1999. Antifungal agents: mode of action, mechanisms of resistance, and correlation of these mechanisms with bacterial resistance. Clin. Microbiol. Rev. 12, 501-517.

Ghasemi Pirbalouti, A., Hashemi, M., Ghahfarokhi, F.T., 2013. Essential oil and chemical compositions of wild and cultivated Thymus daenensis celak and Thymus vulgaris L. Ind. Crops Prod. 48, 43-48.

Giada, M.D.L.R., 2013. Food phenolic compounds: main classes, sources and their antioxidant power. In: Morales-González, J.A. (Ed.), Oxidative Stress and Chronic Degenerative Diseases - A Role for Antioxidants. InTech, Rio de Janeiro - RJ, p. 501, Chapters.

Greenberg, M.S., Glick, M., 2003. Burket's Oral Medicine: Diagnosis and Treatment, 10 Edition. B.C. Decker Inc., USA

Gubbins, P.O., Anaissie, E.J., 2009. Antifungal therapy. In: Livingstone, C. (Ed.), Clinical Mycology. Elsevier, pp. 165-199.

Halberstein, R.A., 2005. Medicinal plants: historical and cross-cultural usage patterns. Ann. Epidemiol. 15, 686-699.

Hashem, M., 2011. Antifungal properties of crude extracts of five Egyptian medicinal plants against dermatophytes and emerging fungi. Mycopathologia 172, 37-46.

Hassawi, D., Kharma, A., 2006. Antimicrobial activity of some medicinal plants against Candida albicans. J. Biol. Sci. 6, 109-114.

Höfling, J.F., Mardegan, R.C., Anibal, P.C., Furletti, V.F., Foglio, M.A., 2011. Evaluation of antifungal activity of medicinal plant extracts against oral Candida albicans and proteinases. Mycopathologia 172 $117-124$.

Inbaraj, J.J., Kukielczak, B.M., Bilski, P., He, Y.-Y., Sik, R.H., Chignell, C.F., 2006. Photochemistry and photocytotoxicity of alkaloids from goldenseal (Hydrastis canadensis L.) 2. Palmatine, hydrastine, canadine, and hydrastinine. Chem. Res. Toxicol. 19, 739-744

Inbaraj, J.J., Kukielczak, B.M., Bilski, P., Sandvik, S.L., Chignell, C.F., 2001 Photochemistry and photocytotoxicity of alkaloids from goldenseal (Hydrastis canadensis L.) 1. Berberine. Chem. Res. Toxicol. 14, 1529-1534.

Irshad, Shreaz, S., Manzoor, N., Khan, L.A., Rizvi, M.M.A., 2011. Anticandidal activity of Cassia fistula and its effect on ergosterol biosynthesis. Pharm. Biol. 49, $727-733$.

Ishaq, M.S., Hussain, M.M., Afridi, M.S., Ali, G., Khattak, M., Ahmad, S., Shakirullah 2014. In vitro phytochemical, antibacterial, and antifungal activities of leaf, stem, and root extracts of Adiantum capillus veneris. Sci. World J., 1-7.

Isolauri, E., Kirjavainen, P.V., Salminen, S., 2002. Probiotics: a role in the treatment of intestinal infection and inflammation. Gut 50, 54-59.

Jainkittivong, A., Butsarakamruha, T., Langlais, R.P., 2009. Antifungal activity of Morinda citrifolia fruit extract against Candida albicans. Oral Surg. Oral Med. Oral Pathol. Oral Radiol. Endod. 108, 394-398.

Jasim, R.M., Al-khaliq, I.M.A., 2011. Inhibitory effect of aqueous Salvia officinalis's leaves in the growth of Candida albicans from infected women with vaginal candidiasis. Al-Kindy Coll. Med. J. 6, 47-49.

Joe, M.M., Jayachitra, J., Vijayapriya, M., 2009. Antimicrobial activity of some common spices against certain human pathogens. J. Med. Plants Res 3, 1134-1136

Jung, H.J., Hwang, I.A., Sung, W.S., Kang, H., Kang, B.S., Seu, Y.B., Lee, D.G., 2005. Fungicidal effect of resveratrol on human infectious fungi. Arch. Pharm. Res. 28, 557-560.

Junio, H.A., Sy-Cordero, A.A., Ettefagh, K.A., Burns, J.T., Micko, K.T., Graf, T.N., Richter, S.J., Cannon, R.E., Oberlies, N.H., Cech, N.B., 2011. Synergy-directed fractionation of botanical medicines: a case study with goldenseal (Hydrastis canadensis). J. Nat. Prod. 74, 1621-1629.

Kaewpiboon, C., Lirdprapamongkol, K., Srisomsap, C., Winayanuwattikun, P., Yongvanich, T., Puwaprisirisan, P., Svasti, J., Assavalapsakul, W., 2012. Studies of the in vitro cytotoxic, antioxidant, lipase inhibitory and antimicrobial activities of selected Thai medicinal plants. BMC Complement. Altern. Med. 12, $1-8$

Kanafani, Z.A., Perfect, J.R., 2008. Resistance to antifungal agents: mechanisms and clinical impact. Clin. Infect. Dis. - Antimicrob. Resist. 46, 120-128.

Kariba, R.M., Siboe, G.M., Dossaji, S.F., 2001. In vitro antifungal activity of Schizozygia coffaeoides bail. (Apocynaceae) extracts. J. Ethnopharmacol. 74, 41-44.

Karomi, M.F., Huda, S.A., Kadhim, S.I., Salman, Z.O., 2012. Effect of methanolic extracts of different parts of Liquorice Glycyrrhiza glabra L. on growth of some pathogenic bacteria and fungus Candida albicans. Iraq Acad. Sci. J. 4, 140-150.

Kaur, I.P., Kuhad, A., Garg, A., Chopra, K., 2009. Probiotics: delineation of prophylactic and therapeutic benefits. J. Med. Food 12, 219-235. 
Kim, J., Sudbery, P., 2011. Candida albicans, a major human fungal pathogen. J. Microbiol. 49, 171-177.

Kim, Y., Lee, H., 2012. Anticandidal effect of Syzygium aromaticum on biofilm formation, cell surface hydrophobicity, and cell cycle. J. Med. Plants Res. 6, 1926-1934.

Kontoyiannis, D.P., Lewis, R.E., 2002. Antifungal drug resistance of pathogenic fungi. Lancet 359, 1135-1144.

Korukluoglu, M., Gurbuz, O., Sahan, Y., Yigit, A., Kacar, O., Rouseff, R., 2008. Chemical characterization and antifungal activity of Origanum onites L. essential oils and extracts. J. Food Saf. 29, 144-161.

Kosalec, I., Pepeljnjak, S., Kustrak, D., 2005. Antifungal activity of fluid extract and essential oil from anise fruits (Pimpinella anisum L., Apiaceae). Acta Pharm. 55, 377-385.

Kostić, D.A., Velicković, J.M., Mitić, S.S., Mitić, M.N., Randelović, S.S., 2012. Phenolic content, and antioxidant and antimicrobial activities of Crataegus oxyacantha $\mathrm{L}$ (Rosaceae) fruit extract from southeast serbia. Trop. J. Pharm. Res. 11, 117-124.

Krisch, J., Ördögh, L., Galgóczy, L., Papp, T., Vágvölgyi, C., 2008. Anticandidal effect of berry juices and extracts from Ribes species. Cent. Eur. J. Biol. 4, 86-89.

Ksouri, R., Ksouri, W.M., Jallali, I., Debez, A., Magné, C., Hiroko, I., Abdelly, C., 2012. Medicinal halophytes: potent source of health promoting biomolecules with medical, nutraceutical and food applications. Crit. Rev. Biotechnol. 32 289-326.

Kuete, V., Poumale, H.M.P., Guedem, A.N., Shiono, Y., Randrianasolo, R., Ngadjui, B.T., 2010. Antimycobacterial, antibacterial and antifungal activities of the methanol extract and compounds from Thecacoris annobonae (Euphorbiaceae). South Afr. J. Bot. 76, 536-542.

Kurdelas, R.R., Lima, B., Tapia, A., Feresin, G.E., Gonzalez Sierra, M., Rodríguez, M.V., Zacchino, S., Enriz, R.D., Freile, M.L., 2010. Antifungal activity of extracts and prenylated coumarins isolated from Baccharis darwinii Hook \& Arn. (Asteraceae). Molecules 15, 4898-4907.

Kursat, M., Emre, I., Yılmaz Ö, Erecevit, P., 2011. Antioxidant and antimicrobial activity in the seeds of Origanum vulgare L. subs. gracile (C. Koch) Ietswaart and Origanum acutidens (Hand. -Mazz.) Ietswaart from Turkey. Grasas Aceites 62, 410-417.

Kwamin, F., Nartey, N.O., Codjoe, F.S., Newman, M.J., 2013. Distribution of Candida species among HIV-positive patients with oropharyngeal candidiasis in Accra, Ghana. J. Infect. Dev. Ctries 7, 41-45.

Lamb, D., Kelly, D., Kelly, S., 1999. Molecular aspects of azole antifungal action and resistance. Drug Resist. Updat. 2, 390-402.

Latte, K.P., Kolodziej, H., 2000. Antifungal effects of hydrolysable tannins and related compounds on dermatophytes, mould fungi and yeasts. Zeitschrift Naturforsch. C - A J. Biosci. 55, 467-472.

Lee, D.G., Park, Y., Kim, M.-R., Jung, H.J., Seu, Y.B., Hahm, K.-S., Woo, E.-R., 2004. Anti-fungal effects of phenolic amides isolated from the root bark of Lycium chinense. Biotechnol. Lett. 26, 1125-1130.

Lewis, L.E., Bain, J.M., Lowes, C., Gow, N.A.R., Erwig, L.-P., 2012. Candida albicans infection inhibits macrophage cell division and proliferation. Fungal Genet. Biol. 49, 679-680.

Li, S.Y., Yang, Y.L., Chen, K.W., Cheng, H.H., Chiou, C.S., Wang, T.H., Lauderdale, T.L Hung, C.C., Lo, H.J., 2006. Molecular epidemiology of long-term colonization of Candida albicans strains from HIV-infected patients. Epidemiol. Infect. 134, 265-269.

Ličina, B.Z., Stefanović, O.D., Vasić, S.M., Radojević, I.D., Dekić, M.S., Čomić, L.R., 2013. Biological activities of the extracts from wild growing Origanum vulgare L. Food Control 33, 498-504.

Lim, S.H., Darah, I., Jain, K., 2006. Antimicrobial activities of tannins extracted from Rhizophora apiculata barks. J. Trop. For. Sci. 18, 59-66.

Liu, X.P., Fan, S.R., Bai, F.Y., Li, J., Liao, Q.P., 2009. Antifungal susceptibility and genotypes of Candida albicans strains from patients with vulvovaginal candidiasis. Mycoses 52, 24-28.

Lott, T.J., Fundyga, R.E., Kuykendall, R.J., Arnold, J., 2005. The human commensal yeast, Candida albicans, has an ancient origin. Fungal Genet. Biol. 42, 444-451.

Lu, Q., Jayatilake, J.A.M.S., Samaranayake, L.P., Jin, L., 2006. Hyphal invasion of Candida albicans inhibits the expression of human beta-defensins in experimental oral candidiasis. J. Invest. Dermatol. 126, 2049-2056.

Lubian, C.T., Teixeira, J.M., Lund, R.G., Nascente, P.S., Del Pino, F.A.B., 2010. Antifungal activity of the aqueous extract from Arctium minus (Hill) Bernh. (Asteraceae) on oral Candida species. Braz. J. Med. Plants 12, 157-162.

Lupetti, A., Danesi, R., Campa, M., Del Tacca, M., Kelly, S., 2002. Molecular basis of resistance to azole antifungals. Trends Mol. Med. 8, 76-81.

Lupetti, A., Nibbering, P.H., Campa, M., Del Tacca, M., Danesi, R., 2003. Molecular targeted treatments for fungal infections: the role of drug combinations. Trends Mol. Med. 9, 269-276.

Madhumitha, G., Saral, A.M., 2011. Preliminary phytochemical analysis, antibacterial, antifungal and anticandidal activities of successive extracts of Crossandra infundibuliformis. Asian Pac. J. Trop. Med. 4, 192-195.

Manayi, A., Saeidnia, S., Faramarzi, M.A., Samadi, N., Jafari, S., Vazirian, M., Ghaderi, A., Mirnezami, T., Hadjiakhoondi, A., Ardekani, M.R.S., Khanavi, M., 2013. A comparative study of anti-Candida activity and phenolic contents of the calluses from Lythrum salicaria L. in different treatments. Appl. Biochem. Biotechnol. 170, 176-184.

Mandeel, Q., Taha, A., 2005. Assessment of in vitro antifungal activities of various extracts of indigenous bahraini medicinal plants. Pharm. Biol 43, 340-348.

Martins, N., Barros, L., Santos-Buelga, C., Henriques, M., Silva, S., Ferreira, I.C.F.R., 2015. Evaluation of bioactive properties and phenolic compounds in different extracts prepared from Salvia officinalis L. Food Chem. 170, 378-385.
Martins, N., Ferreira, I.C.F.R., Barros, L., Silva, S., Henriques, M., 2014. Candidiasis: predisposing factors, prevention, diagnosis and alternative treatment. Mycopathologia 177, 223-240.

Masoko, P., Mmushi, T.J., Mogashoa, M.M., Mokgotho, M.P., Mampuru, L.J., Howard, R.L., 2008. In vitro evaluation of the antifungal activity of Sclerocarya birrea extracts against pathogenic yeasts. Afr. J. Biotechnol 7, 3521-3526.

Masoko, P., Picard, J., Howard, R.L., Mampuru, L.J., Eloff, J.N., 2010. In vivo antifungal effect of Combretum and Terminalia species extracts on cutaneous wound healing in immunosuppressed rats. Pharm. Biol. 48, 621-632.

Mathur, A., Singh, R., Yousuf, S., Bhardwaj, A., Verma, S.K., Babu, P., Gupta, V., Prasad, G.B.K.S., Dua, V.K., 2011. Antifungal activity of some plant extracts against clinical pathogens. Adv. Appl. Sci. Res. 2, 260-264.

Maurya, I.K., Thota, C.K., Sharma, J., Tupe, S.G., Chaudhary, P., Singh, M.K., Thakur I.S., Deshpande, M., Prasad, R., Chauhan, V.S., 2013. Mechanism of action of novel synthetic dodecapeptides against Candida albicans. Biochim. Biophys. Acta 1830, 5193-5203.

Mayer, F.L., Wilson, D., Hube, B., 2013. Candida albicans pathogenicity mechanisms Virulence 4, 119-128.

McCullough, M.J., Ross, B.C., Reade, P.C., 1996. Candida albicans: a review of its history, taxonomy, epidemiology, virulence attributes, and methods of strain differentiation. Int. J. Oral Maxillofac. Surg. 25, 136-144.

Menezes, T.O.A., Alves, A.C.B.A., Vieira, J.M.S., Menezes, S.A.F., Alves, B.P., Mendonça, L.C.V., 2009. In vitro evaluation of the antifungal activity of essential oils and plant extracts from amazonian region against Candida albicans. J. Odontol. UNESP 38, 184-191.

Modaressi, M., Shahsavari, R., Ahmadi, F., Rahimi-Nasrabadi, M., Abiri, R., Mikaeli, A., Batoli, H., 2013. The evaluation of antibacterial, antifungal and antioxidant activity of methanolic extract of Mindium laevigatum (Vent.) Rech. F., from central part of iran. Jundishapur J. Nat. Pharm. Prod. 8, 34-40.

Murray, M.T., 2004. The Healing Power Of Herbs, 2nd ed. Random House, New York, NY.

Murray, M.T., Pizzorno, J., 1998. Enciclopedia de Medicina Natural, 2nd ed. Ediciones Tutor S.A., Espanha.

Nelson, D.L., Cox, M.M., 2000. Lehninger Principles Of Biochemistry, Third ed. Worth Publishers, Inc., New York.

Ngameni, B., Kuete, V., Simo, I.K., Mbaveng, A.T., Awoussong, P.K., Patnam, R., Roy, R., Ngadjui, B.T., 2009. Antibacterial and antifungal activities of the crude extract and compounds from Dorstenia turbinata (Moraceae). South Afr. J. Bot. $75,256-261$.

Niño, J., Narváez, D.M., Mosquera, O.M., Correa, Y.M., 2007. Antibacterial, antifungal and cytotoxic activities of eight asteraceae and two Rubiaceae plants from Colombian biodiversity. Braz. J. Microbiol., 566-570.

Noël, T., 2012. The cellular and molecular defense mechanisms of the Candida yeasts against azole antifungal drugs. J. Mycol. Med. 22, 173-178.

Nordin, M.-A.-F., Harun, W.H.A.W., Razak, F.A., 2013. Antifungal susceptibility and growth inhibitory response of oral Candida species to Brucea javanica Linn. extract. BMC Complement. Altern. Med. 13, 1-8.

Ozçelik, B., Kartal, M., Orhan, I., 2011. Cytotoxicity, antiviral and antimicrobial activities of alkaloids, flavonoids, and phenolic acids. Pharm. Biol. 49, 396-402.

Papageorgiou, V., Gardeli, C., Mallouchos, A., Papaioannou, M., Komaitis, M., 2008. Variation of the chemical profile and antioxidant behavior of Rosmarinus officinalis L. And Salvia fruticosa Miller grown in greece. J. Agric. Food Chem. 56 7254-7264

Parekh, J., Chanda, S., 2008. In vitro antifungal activity of methanol extracts of some indian medicinal plants against pathogenic yeast and moulds. Afr. J. Biotechnol. 7, 4349-4353.

Parsaeimehr, A., Sargsyan, E., Javidnia, K., 2010. A comparative study of the antibacterial, antifungal and antioxidant activity and total content of phenolic compounds of cell cultures and wild plants of three endemic species of Ephedra. Molecules 15, 1668-1678.

Pathak, A.K., 2012. Anti-Candida activity of aqueous extracts of some herbals. Indian J. Fundam. Appl Life Sci. 2, 1-6.

Pendota, S.C., Aderogba, M.A., Ndhlala, A.R., Staden, J.V., 2013. Antimicrobial and acetylcholinesterase inhibitory activities of Buddleja salviifolia (L.) Lam. leaf extracts and isolated compounds. J. Ethnopharmacol. 148, 515-520.

Pereira Gonzales, F., Maisch, T., 2012. Photodynamic inactivation for controlling Candida albicans infections. Fungal Biol. 116, 1-10.

Perlin, D.S., 2009. Resistance to echinocandin-class antifungal drugs. Drug Resist. Updat. 10, 121-130.

Perlin, D.S., 2014. Current perspectives on echinocandin class drugs. Future Microbiol. 6, 441-457.

Pessini, G.L., Filho, B.P.D., Nakamura, C.V., Cortez, D.A.G., 2005. Antifungal activity of the extracts and neolignans from Piper regnellii (Miq.) C. DC. var. pallescens (C. DC.) Yunck. J. Braz. Chem. Soc. 16, 1130-1133.

Petrovska, B.B., 2012. Historical review of medicinal plants' usage. Pharmacogn. Rev, 6, 1-5.

Picerno, P., Mencherini, T., Sansone, F., Gaudio, P., Del, Granata, I., Porta, A., Aquino, R.P., 2011. Screening of a polar extract of Paeonia rockii: composition and antioxidant and antifungal activities. J. Ethnopharmacol. 138, $705-712$.

Pierce, G.E., 2005. Pseudomonas aeruginosa, Candida albicans, and device-related nosocomial infections: implications, trends, and potential approaches for control. J. Ind. Microbiol. Biotechnol. 32, 309-318.

Politeo, O., Juki, M., Milo, M., 2006. Chemical composition and antioxidant activity of essential oils of twelve spice plants. Croat. Chem. Acta 79, 545-552. 
Prabhakar, K., Kumar, L.S., Rajendran, S., Chandrasekaran, M., Bhaskar, K., Sajit Khan, A.K., 2008. Antifungal activity of plant extracts against Candida species from oral lesions. Indian J. Pharm. Sci. 70, 801-803.

Proença da Cunha, A., Silva, A.P., Roque, O.R., 2012. Plantas e produtos vegetais em Fitoterapia, 4th ed. Lisboa.

Proença da Cunha, A., Teixeira, F., Silva, A.P., Roque, O.R., 2010. Plantas na terapêutica: farmacologia e ensaios clinicos, 2nd ed. Lisboa.

Qader, M.K., Khalid, N.S., Saadullah, A.A.M., 2013. Screening of some plant extracts for antifungal activity against Candida species. Int. J. Microbiol. Immunol. Res. 1, 65-67.

Rajeh, M.A.B., Zuraini, Z., Sasidharan, S., Latha, L.Y., Amutha, S., 2010. Assessment of Euphorbia hirta L. leaf, flower, stem and root extracts for their antibacterial and antifungal activity and brine shrimp lethality. Molecules 15, 6008-6018.

Raman, S.B., Nguyen, M.H., Cheng, S., Badrane, H., Iczkowski, K.A., Wegener, M., Gaffen, S.L., Mitchell, A.P., Clancy, C.J., 2013. A competitive infection model of hematogenously disseminated candidiasis in mice redefines the role of Candida albicans IRS4 in pathogenesis. Infect. Immun. 81, 1430-1438.

Rana, I.S., Rana, A.S., Rajak, R.C., 2011. Evaluation of antifungal activity in essential oil of the Syzygium aromaticum (L.) by extration, purification and analysis of its main component eugenol. Braz. J. Microbiol. 42, 1269-1277.

Ren, B., Xia, B., Li, W., Wu, J., Zhang, H., 2009. Two novel phenolic compounds from Stenoloma chusanum and their antifungal activity. Chem. Nat. Compd. 45, 182-186.

Rice-Evans, C.A., Miller, N.J., Paganga, G., 1996. Structure-antioxidant activity relationships of flavonoids and phenolic acids. Free Radic. Biol. Med. 20, 933-956.

Robbins, R.J., 2003. Phenolic acids in foods: an overview of analytical methodology J. Agric. Food Chem. 51, 2866-2887.

Rose, J., 1999. Herbal and nutritional support for the immune system. Clin. Nutr. Insights 6, 1-4

Rubió, L., Motilva, M.-J., Romero, M.-P., 2013. Recent advances in biologically active compounds in herbs and spices: a review of the most effective antioxidant and anti-inflammatory active principles. Crit. Rev. Food Sci. Nutr. 53, 943-953.

Saeed, S., Tariq, P., 2007. Antimicrobial activities of Emblica officinalis and Coriandrum sativum against gram positive bacteria and Candida albicans. Pakistan J. Bot. 39, 913-917.

Safaei-Ghomi, J., Ahd, A.A., 2010. Antimicrobial and antifungal properties of the essential oil and methanol extracts of eucalyptus largiflorens and Eucalyptus intertexta. Pharmacogn. Mag. 6, 172-175.

Saleem, M., Nazir, M., Ali, M.S., Hussain, H., Lee, Y.S., Riaz, N., Jabbar, A., 2010. Antimicrobial natural products: an update on future antibiotic drug candidates. Nat. Prod. Rep. 27, 238-254.

Sangamwar, A.T., Deshpande, U.D., Pekamwar, S.S., 2008. Antifungals: need to search for a new molecular target. Indian J. Pharm. Sci 70, 423-430.

Sanglard, D., 2002. Resistance of human fungal pathogens to antifungal drugs. Curr Opin. Microbiol. 5, 379-385.

Sanglard, D., Odds Reviews, F.C., 2002. Resistance of candida species to antifungal agents: molecular mechanisms and clinical consequences. Lancet Infect. Dis. 2 $73-85$.

Santos-Buelga, C., Gonzalez-Manzano, S., Dueñas, M., Gonzalez-Paramas, A.M., 2012. Extraction and isolation of phenolic compounds. Methods Mol. Biol. 864, 427-464.

Sardi, J.C.O., Scorzoni, L., Bernardi, T., Fusco-Almeida, A.M., Giannini, M.J.S.M., 2013. Candida species: current epidemiology, pathogenicity, biofilm formation, natural antifungal products and new therapeutic options. J. Med. Microbiol. 62, $10-24$.

Sertel, S., Fu, Y., Zu, Y., Rebacz, B., Konkimalla, B., Plinkert, P.K., Krämer, A., Gertsch, J., Efferth, T., 2011. Molecular docking and pharmacogenomics of Vinca alkaloids and their monomeric precursors, vindoline and catharanthine. Biochem. Pharmacol. 81, 723-735.

Shamim, S., Ahmed, S.W., Azhar, I., 2004. Antifungal activity of Allium, Aloe, and Solanum species. Arch. Physiol. Biochem. 42, 491-498.

Sher, A., 2009. Antimicrobial activity of natural products from medicinal plants. Gomal J. Med. Sci. 7, 72-78.

Shojaii, A., Fard, M.A., 2012. Review of pharmacological properties and chemical constituents of Pimpinella anisum. Int. Sch. Res. Netw. - ISRN Pharm. 2012, 1-8.

Siler, B., Živković, S., Banjanac, T., Cvetković, J., Živković, J.N., Cirić, A., Soković, M., Mišić, D., 2014. Centauries as underestimated food additives: antioxidant and antimicrobial potential. Food Chem. 147, 367-376.

Silva, F., Ferreira, S., Duarte, A., Mendonça, D.I., Domingues, F.C., 2011a. Antifungal activity of Coriandrum sativum essential oil, its mode of action against Candida species and potential synergism with amphotericin B. Phytomedicine 19, $42-47$.

Silva, S., Henriques, M., Hayes, A., Oliveira, R., Azeredo, J., Williams, D.W., 2011b. Candida glabrata and Candida albicans co-infection of an in vitro oral epithelium. J. Oral Pathol. Med. 40, 421-427.

de Silva, G.S., 2012. Study of the antimicrobial activity of the medicinal plant extracts against clinical isolates of Candida species. State University of Paraíba.

Silva, N.C.C., Fernandes Júnior, A., 2010. Biological properties of medicinal plants: a review of their antimicrobial activity. J. Venom. Anim. Toxins Incl. Trop. Dis. 16, 402-413.

Singh, A., Duggal, S., Kaur, N., Singh, J., 2010. Berberine: alkaloid with wide spectrum of pharmacological activities. J. Nat. Prod. 3, 64-75.

Singh, R.P., Sharad, S., Kapur, S., 2004. Free radicals and oxidative stress in neurodegenerative diseases: relevance of dietary antioxidants. J. Indian Acad. Clin. Med. 5, 218-225.
Sobel, J.D., 2007. Vulvovaginal candidosis. Lancet 369, 1961-1971.

Souza, N.A.B., Lima, de, E.O., Guedes, D.N., Pereira, F.D.O., Souza, E.L., de, Sousa, F.B. 2010. Efficacy of Origanum essential oils for inhibition of potentially pathogenic fungi. Braz. J. Pharm. Sci. 46, 499-508.

Sule, A., Ahmed, Q.U., Latip, J., Samah, O.A., Omar, M.N., Umar, A., Dogarai, B.B.S., 2012. Antifungal activity of Andrographis paniculata extracts and active principles against skin pathogenic fungal strains in vitro. Pharm. Biol. 50, 850-856.

Sullivan, D.J., Moran, G.P., Pinjon, E., Almosaid, A., Stokes, C., Vaughan, C., Coleman, D.C., 2004. Comparison of the epidemiology, drug resistance mechanisms, and virulence of Candida dubliniensis and Candida albicans. FEMS Yeast Res. 4, 369-376.

Sun, S., Lou, H., Gao, Y., Fan, P., Ma, B., Ge, W., Wang, X., 2004. Liquid chromatography-tandem mass spectrometric method for the analysis of fluconazole and evaluation of the impact of phenolic compounds on the concentration of fluconazole in Candida albicans. J. Pharm. Biomed. Anal. 34, 1117-1124.

Tarry, W., Fisher, M., Shen, S., Mawhinney, M., 2005. Candida albicans: the estrogen target for vaginal colonization. J. Surg. Res. 129, 278-282.

Tharkar, P.R., Tatiya, A.U., Shinde, P.R., Surana, S.J., Patil, U.K., 2010. Antifungal activity of Glycyrrhiza glabra Linn. and Emblica officinalis Gaertn. by direct bioautography method. Int. J. PharmTech. Res. 2, 1547-1549.

Thirach, S., Tragoolpua, K., Punjaisee, S., Jatisatienr, C., Kharmwan, C., Kunyanone, N., 2003. Antifungal activity of some medicinal plant extracts against Candida albicans and Cryptococcus neoformans. Acta Hortic. 597, 217-221.

Timothy, S.Y., Wazis, C.H., Adati, R.G., Maspalma, I.D., 2012. Antifungal activity of aqueous and ethanolic leaf extracts of CCassia alata Linn. J. Appl. Pharm. Sci. 2 182-185.

Tintino, S.R., Souza, C.E.S., Guedes, G.M.M., Costa, J.I.V., Duarte, F.M., Chaves, M.C.O., Silva, V.A., Pessôa, H.L.F., Lima, M.A., Garcia, C.A., Coutinho, H.D.M., 2014. Modulatory antimicrobial activity of piper arboreum extracts. Acta Bot. Croat. 73, 281-289.

Tomczykowa, M., Tomczyk, M., Jakoniuk, P., Tryniszewska, E., 2008. Antimicrobia and antifungal activities of the extracts and essential oils of bidens tripartita. Folia Histochem. Cytobiol. 46, 389-393.

Touré, A., Bahi, C., Ouattara, K., Djama, J.A., Coulibaly, A., 2011. Phytochemical screening and in vitro antifungal activities of extracts of leaves of Morinda morindoides (Morinda, Rubiaceae). J. Med. Plants Res. 5, 6780-6786.

Tsai, P.-W., Chen, Y.-T., Hsu, P.-C., Lan, C.-Y., 2013. Study of Candida albicans and its interactions with the host: a mini review. Biomedicine 3, 51-64.

Tsuzuki, J.K., Svidzinski, T.I.E., Shinobu, C.S., Silva, L.F.A., Rodrigues-Filho, E., Cortez D.A.G., Ferreira, I.C.P., 2007. Antifungal activity of the extracts and saponins from Sapindus saponaria L. Ann. Braz. Acad. Sci. 79, 577-583.

Unver, A., Arslan, D., Cetynkaya, Z., Ozcan, M.M., 2008. Antimycotic activity of methanol extracts of sage (Salvia officinalis L.), laurel (Laurus nobilis L.) and thyme (Thymbra spicata L.). J. Essent. Oil Bear. Plants 11, 90-95.

Valko, M., Leibfritz, D., Moncol, J., Cronin, M.T.D., Mazur, M., Telser, J., 2007. Free radicals and antioxidants in normal physiological functions and human disease. Int. J. Biochem. Cell Biol. 39, 44-84.

Vázquez-González, D., Perusquía-Ortiz, A.M., Hundeiker, M., Bonifaz, A., 2013. Opportunistic yeast infections: candidiasis, cryptococcosis, trichosporonosis and geotrichosis. J. Ger. Soc. Dermatol. 11, 381-394.

Volleková, A., Kost'álová, D., Kettmann, V., Tóth, J., 2003. Antifungal activity of Mahonia aquifolium extract and its major protoberberine alkaloids. Phyther Res. 17, 834-837.

Wächtler, B., Citiulo, F., Jablonowski, N., Förster, S., Dalle, F., Schaller, M., Wilson, D., Hube, B., 2012. Candida albicans-epithelial interactions: dissecting the roles of active penetration, induced endocytosis and host factors on the infection process. PLoS One 7, 1-10.

Walch, S.G., Tinzoh, L.N., Zimmermann, B.F., Stühlinger, W., Lachenmeier, D.W., 2011. Antioxidant capacity and polyphenolic composition as quality indicators for aqueous infusions of Salvia officinalis L. (sage tea). Front. Pharmacol. 2, 1-6.

Waldorf, A.R., Polak, A., 1983. Mechanisms of action of 5-fluorocytosine. Antimicrob. Agents Chemother. 23, 79-85.

Wang, J., Lou, J., Luo, C., Zhou, L., Wang, M., Wang, L., 2012. Phenolic compounds from Halimodendron halodendron (Pall.) Voss and their antimicrobial and antioxidant activities. Int. J. Mol. Sci. 13, 11349-11364.

Webster, D., Taschereau, P., Belland, R.J., Sand, C., Rennie, R.P., 2008. Antifungal activity of medicinal plant extracts; preliminary screening studies. J. Ethnopharmacol. 115, 140-146.

Westwater, C., Schofield, D.A., Nicholas, P.J., Paulling, E.E., Balish, E., 2007. Candida glabrata and Candida albicans; dissimilar tissue tropism and infectivity in a gnotobiotic model of mucosal candidiasis. FEMS Immunol. Med. Microbiol. 51, 134-139.

White, T.C., Holleman, S., Dy, F., Laurence, F., Stevens, D.A., Mirels, L.F., 2002. Resistance mechanisms in clinical isolates of Candida albicans. Antimicrob. Agents Chemother. 46, 1704-1713.

White, T.C., Marr, K.A., Bowden, R.A., 1998. Clinical, cellular, and molecular factors that contribute to antifungal drug resistance. Clin. Microbiol. Rev. 11 382-402.

Wojdylo, A., Oszmianski, J., Czemerys, R., 2007. Antioxidant activity and phenolic compounds in 32 selected herbs. Food Chem. 105, 940-949.

Wong, K.S., Tsang, W.K.P., 2009. In vitro antifungal activity of the aqueous extract of Scutellatia baicalensis georgi root against Candida albicans. Int. J. Antimicrob. Agents 34, 283-284. 
Wyk, C., Van, Botha, F.S., Steenkamp, V., 2009. In vitro antimicrobial activity of medicinal plants against oral Candida albicans isolates. Int. J. Biomed. Pharm. Sci. 3, 26-30.

Xie, Q., Johnson, B.R., Wenckus, C.S., Fayad, M.I., Wu, C.D., 2012. Efficacy of berberine, an antimicrobial plant alkaloid, as an endodontic irrigant against a mixed-culture biofilm in an in vitro tooth model. J. Endod. 38, 1114-1117.

Yang, S.W., Ubillas, R., McAlpine, J., A, Stafford, Ecker, D.M., Talbot, M.K., Rogers, B. 2001. Three new phenolic compounds from a manipulated plant cell culture, Mirabilis jalapa. J. Nat. Prod. 64, 313-317.
Yazdani, D.M., Rezazadeh, S., Amin, G., Abidin, M.Z., Shahnazi, S., Jamalifar, H., 2009. Antifungal activity of dried extracts of anise (Pimpinella anisum L.) and star anise (Illicium verum Hook.) against dermatophyte and saprophyte fungi. J Med. Plants 8, 24-29.

Zhu, X.F., Zhang, H.X., Lo, R., 2005. Antifungal activity of Cynara scolymus L. extracts. Fitoterapia 76, 108-111. 